\title{
Maximizing TADF via Conformational Optimization
}

Changhae Andrew Kim, Troy Van Voorhis

Submitted date: 05/05/2021 Posted date: 07/05/2021

Licence: CC BY-NC-ND 4.0

Citation information: Kim, Changhae Andrew; Van Voorhis, Troy (2021): Maximizing TADF via Conformational Optimization. ChemRxiv. Preprint. https://doi.org/10.26434/chemrxiv.14529726.v1

We investigate a new strategy to enhance thermally activated delayed fluorescence (TADF) in organic light-emitting diodes (OLEDs). Given that the TADF rate of a molecule depends on its conformation, we hypothesize that there exists a conformation that maximizes the TADF rate. In order to test this idea, we use time-dependent density functional theory (TD-DFT) to simulate the TADF rates of several TADF emitters, while shifting their geometries towards higher TADF rates in a select subspace of internal coordinates. We find that geometric changes in this subspace can increase the TADF rate up to three orders of magnitude with respect to the minimum energy conformation, and the simulated TADF rate can even be brought into the submicrosecond timescales under the right conditions. Furthermore, the rate enhancement can be maintained with a conformational energy that are within the reach of modern synthetic chemistry. Analyzing the maximum TADF conformation, we extract a number of structural motifs that might provide a useful handle on the TADF rate of a donor-acceptor (DA) system. The incorporation of conformational engineering into the TADF technology could usher in a new paradigm of OLEDs.

File list (2)

manuscript.pdf (7.03 MiB)

view on ChemRxiv • download file

si.pdf (18.22 MiB)

view on ChemRxiv - download file 


\title{
Maximizing TADF via Conformational Optimization
}

\author{
Changhae Andrew Kim and Troy Van Voorhis* \\ Department of Chemistry, Massachusetts Institute of Technology, Cambridge, \\ Massachusetts 02139, United States \\ E-mail: tvan@mit.edu
}

\begin{abstract}
We investigate a new strategy to enhance thermally activated delayed fluorescence (TADF) in organic light-emitting diodes (OLEDs). Given that the TADF rate of a molecule depends on its conformation, we hypothesize that there exists a conformation that maximizes the TADF rate. In order to test this idea, we use time-dependent density functional theory (TD-DFT) to simulate the TADF rates of several TADF emitters, while shifting their geometries towards higher TADF rates in a select subspace of internal coordinates. We find that geometric changes in this subspace can increase the TADF rate up to three orders of magnitude with respect to the minimum energy conformation, and the simulated TADF rate can even be brought into the submicrosecond timescales under the right conditions. Furthermore, the rate enhancement can be maintained with a conformational energy that are within the reach of modern synthetic chemistry. Analyzing the maximum TADF conformation, we extract a number of structural motifs that might provide a useful handle on the TADF rate of a donor-acceptor (DA) system. The incorporation of conformational engineering into the TADF technology could usher in a new paradigm of OLEDs.
\end{abstract}




\section{Introduction}

Organic light-emitting diodes (OLEDs) are a promising solution in digital displays and lighting applications. Since Tang and Van Slyke demonstrated the first practical OLED device in 1987, ${ }^{1}$ OLEDs have attracted widespread research and development (R\&D) efforts in both the academia and industry. OLED displays exhibit supremacy in energy efficiency, image quality, response time, and compactness over conventional technologies, such as liquid crystal displays (LCDs). ${ }^{2-4}$ In addition, OLEDs are expected to help reduce global energy consumption $^{5,6}$ and yield new commercial products, such as transparent lighting panels and flexible displays. ${ }^{7,8}$

A key issue in developing more efficient OLEDs is overcoming the disadvantageous spin statistics. When electrons and holes are injected into the organic layer, they recombine in one of the four possible spin states with equal likelihoods: one singlet state and three triplet states. Whereas the singlet excitons can emit a photon and decay to the likewise singlet ground state, radiative decay of the triplet excitons is spin-forbidden. Most of the exitons are dissipated as heat, and the external quantum efficiency (EQE) of fluorescent OLEDs cannot exceed $25 \%$. As a way to harvest the triplet excitons, Baldo et al. introduced phosphorescent OLEDs (PhOLEDs), which activate the otherwise forbidden transition between the triplet excited state and the singlet ground state. ${ }^{9}$ Using the strong spin-orbit coupling (SOC) in heavy metal complexes, the rate of phosphorescence can be brought into the useful microsecond timescale. ${ }^{7,10}$ However, in spite of the commercial success of red and green PhOLEDs, an efficient and stable blue PhOLED remains out of reach, ${ }^{4,11}$ and the rarity and toxicity of heavy metals are also problematic.

Thermally activated delayed fluorescence (TADF) is an alternative approach of converting the non-emissive triplet excitons to emissive singlet excitons. ${ }^{12,13}$ In typical organic molecules, electron exchange stabilizes the first triplet excited state $\left(T_{1}\right)$ with respect to the first singlet excited state $\left(\mathrm{S}_{1}\right)$. However, if the singlet-triplet $(\mathrm{ST})$ energy gap is less than few $k_{\mathrm{B}} T$, thermal fluctuations can drive the reverse intersystem crossing (RISC) of the $\mathrm{T}_{1}$ population 
into the $\mathrm{S}_{1}$ state. Modulation of the exchange energy can be accomplished by tuning the spatial overlap between the highest occupied molecular orbital (HOMO) and the lowerest unoccupied molecular orbital (LUMO). In the first metal-free TADF OLED, Adachi et al. employed a donor-acceptor (DA) architecture to localize the HOMO and the LUMO on orthogonal moieties, creating $\mathrm{S}_{1}$ and $\mathrm{T}_{1}$ states of charge transfer $(\mathrm{CT})$ character. ${ }^{14,15}$ Since then, a large number of TADF emitters have been designed on the same principle, ${ }^{16-20}$ and high-throughput screening approaches with the aid of machine learning techniques have been employed to give extensive surveys of the relevant chemical space. ${ }^{21,22}$ As a culmination of the R\&D efforts, TADF OLEDs are becoming competitive with commercial PhOLEDs. ${ }^{23-26}$

Unfortunately, spatial separation of the HOMO and the LUMO also diminishes the transition dipole moment $(\mathrm{TDM})$ between the $\mathrm{S}_{1}$ state and the ground state $\left(\mathrm{S}_{0}\right)$. A decrease in the TDM entails a decrease in both the prompt and delayed fluorescence rates. The trade-off between a small ST gap and a large TDM is manifest even in the outcomes of high-throughput screening studies. ${ }^{21,22}$ In addition, the identical CT character of the $\mathrm{S}_{1}$ and $\mathrm{T}_{1}$ states is expected to lead to a vanishing SOC according to El-Sayed's rule ${ }^{27}$ and hence a vanishing RISC rate. However, the trade-off might not be an intrinsic limitation of TADF, but an artifact of the design principles based on an oversimplified model. Indeed, it has been assumed that considering the $\mathrm{S}_{1}$ and $\mathrm{T}_{1}$ states would provide an adequate account of the TADF mechanism, even though recent studies have demonstrated that higher triplet states might have an important role in the RISC step. ${ }^{28-30}$ The two-state model of TADF does not provide the requisite insight to guide the development of trend-breaking emitters.

Recently, a four-state model of TADF has been proposed that might provide a way to overcome the trade-off. ${ }^{31}$ According to the four-state model, the ST gap contains additional terms that can counter the effects of electron exchange, enabling the coexistence of a small ST gap and a large TDM in the same molecule. Furthermore, the adiabatic $\mathrm{S}_{1}$ and $\mathrm{T}_{1}$ wavefunctions contain different fractions of diabatic CT and local excitiation (LE) wavefunctions, enabling the coexistence of a vanishing ST gap and a non-vanishing SOC. Al- 
though the mathematical forms of the $S_{1}$ and $T_{1}$ energies and wavefunctions do not render themselves to straight-forward interpretation, exploring the conformation space of TADF emitters has been proposed as a practical approach to discover new design principles. Using molecular dynamics (MD) to sample the conformation space and time-dependent density functional theory (TD-DFT) to simulate the electronic excitations, de Silva et al. showed that a DA complex can achieve upto an 800-fold enhancement of the TADF rate with respect

to the minimum energy conformation. ${ }^{31}$ Indeed, a number of theoretical studies have shown that the conformation of a molecule can modulate the excitation energies and state-to-state couplings that contribute to the TADF rate. ${ }^{32-34}$ It would be reasonable to believe that the TADF rate has a strong dependence on the conformation.

In this work, we take the idea of conformational dependence to the next step and examine the potential of direct optimization to maximize the TADF rate. Using a number of known TADF emitters, we show that the TADF rate can be enhanced up to three orders of magnitude and can even be brought into the submicrosecond timescales via suitable changes of conformation. The maximum TADF conformation is free of thermal fluctuations that plague MD snapshot geometries, and one can extract specific hints, as well as general principles that can boost the performance of TADF OLEDs. Furthermore, we find that taking a small subset of the conformational variables suffices to gain substantial control over the TADF rate, and the relevant variables seem to overlap with degrees of freedom (DOFs) that might be accessible via steric hindrance or mechanical strain. Hence, conformational optimization appears to herald a new paradigm of TADF OLEDs.

\section{Methods}

The TADF rate was estimated using the formulation of Adachi et al. ${ }^{35}$ First, the rate of TADF is understood to mean the quasi-steady state rate at which the excited states are 
depopulated via fluorescence

$$
k_{\mathrm{TADF}} \equiv \frac{k_{\mathrm{F}}\left[\mathrm{S}_{1}\right]}{\left[\mathrm{S}_{1}\right]+\left[\mathrm{T}_{1}\right]+\left[\mathrm{T}_{2}\right]}
$$

where we have assumed that interal conversion (IC) is fast enough that only the $\mathrm{S}_{1}, \mathrm{~T}_{1}$, and $\mathrm{T}_{2}$ states have significant populations. In our experience, omission of the $\mathrm{T}_{2}$ state can lead to an overestimation of the TADF rate when the $\mathrm{T}_{2}$ energy is below the $\mathrm{S}_{1}$ energy. Assuming that the forward and reverse intersystem crossing (ISC and RISC) are fast compared to the radiative and non-radiative decay processes, the formula reduces to

$$
k_{\mathrm{TADF}}=\frac{k_{\mathrm{F}}}{1+K_{1}+K_{2}}
$$

where $K_{n}$ is the equilibrium constant between the $\mathrm{S}_{1}$ state and the $\mathrm{T}_{\mathrm{n}}$ state

$$
K_{n}=3 \exp \left(\frac{\Delta E_{\mathrm{S}_{1}-\mathrm{T}_{n}}}{k_{B} T}\right)
$$

The fluorescence rate is estimated by the Einstein coefficients ${ }^{36}$

$$
k_{\mathrm{F}}=\frac{e^{2} \omega_{10}^{2}}{2 \pi \epsilon_{0} m_{e} c^{3}} f_{10}
$$

where $e$ and $m_{e}$ are the electron charge and mass, respectively; $c$ is the speed of light; and $\omega_{10}$ and $f_{10}$ are the $\mathrm{S}_{1} \rightarrow \mathrm{S}_{0}$ energy gap and oscillator strength, respectively. In the final objective function, we added a penalty to damp the increase in the conformational energy (the DFT energy with respect to the energy minimum) and byproduct features that might arise in the optimization

$$
\Phi=k_{\mathrm{TADF}}-\Lambda \Delta E_{\mathrm{DFT}}
$$

where $\Lambda$ is a tunable parameter and $\Delta E_{\mathrm{DFT}}$ is the change in the DFT energy with respect to the minimum energy conformation. We chose the tunable parameter to be on the order 
$\Lambda \sim 1 \mathrm{\mu s}^{-1} E_{\mathrm{h}}^{-1}$

We need to emphasize a couple of points regarding the objective function. First, Equation 2 attempts to describe a single conformer of definite geometry. There are theories that enable the inclusion of geometric relaxation (or reogranization) ${ }^{37,38}$ and vibrational effects ${ }^{39}$ in estimating the rates of RISC and fluorescence, and these would be the proper way to describe a dynamical ensemble of conformers. ${ }^{40-42}$ However, the TADF rate maximization is a geometry optimization where we maximize the TADF rate instead of minimizing the energy, which necessitates the evaluation of the TADF rate at a definite geometry. Hence, nuclear motion is incompatible with the present investigation.

Second, Equation 2 represents an upper bound on the TADF rate. In the real universe, non-radiative decay and a finite RISC rate would entail a slowdown in addition to the fluorescence rate and the thermodynamic cap on the $S_{1}$ population. Nonetheless, the formula appeals to our purposes in a number of ways. In the first place, we are only interested in good TADF emitters, where TADF dominates over non-radiative decay, so it makes sense to compute the TADF rate by itself and verify after the fact that TADF proceeds on a feasible timescale. Moreover, Brédas et al. calculated the RISC rates of several TADF emitters and found that RISC rates on the microsecond timescale are not uncommon. ${ }^{30} \mathrm{We}$ expect that RISC would not be a severe bottleneck in most cases and that the formula would provide a tight upper bound on the TADF rate. Indeed, we found that the RISC only makes quantitative corrections and does not alter the qualitative trends in our model systems. The computational details and numerical results have been provided in the SI.

The electronic structure calculations were performed using the B3LYP exchange-correlation functional ${ }^{43-48}$ and the 6-31+G* basis set $^{49-52}$ as implemented in the Q-Chem 5.1 software package. ${ }^{53}$ Although standard hybrid functionals are known to underestimate the energy and overestimate the CT character of CT-like states, ${ }^{54,55}$ our objective was not to make quantitative predictions of TADF rates, but to extract qualitative trends that can guide the design of TADF emitters. Besides, analytical derivatives of the TDM are not available, so 
the TADF rate maximization entails much higher computational costs than the usual energy minimization. Thus, it was desirable to use a functional that can reproduce the qualitative trends at minimal costs.

In order to establish that conformational dependence is not an artifact of B3LYP, the electronic structures at the key geometries were recalculated using PBE0, ${ }^{56-58} \mathrm{M} 06-2 \mathrm{X},{ }^{59}$ and LRC- $\omega^{*} \mathrm{PBE} .{ }^{60-62}$ The asterisk indicates IPEA-tuning of the range-separation parameter, which we did at the minimum energy conformation in vacuum. Solvent effects were examined in the cases of M06-2X and LRC- $\omega^{*} \mathrm{PBE}$, using the integral equation formalism polarizable continuum model (IEF-PCM $)^{63-65}$ and first order, perturbative state-specific $(\mathrm{ptSS})^{66}$ treatment of the excited states. We used the dielectric constant and optical dielectric constant of toluene at room temperature: $\varepsilon=2.379$ and $\varepsilon_{\infty}=2.232 .{ }^{67}$

The images of the geometries were generated in Avogadro 1.2.0, ${ }^{68}$ and the images of the natural transition orbitals (NTOs) ${ }^{69}$ were generated in MacMolPlt 7.7.70

The TADF rate maximization was performed using a variation of the Nelder-Mead simplex method. ${ }^{71,72}$ Since there are multiple versions of the method, we have outlined our algorithm in the SI. The geometries of the molecules were represented in the Z-matrix coordinates. We have included the Z-matrices of the model systems at the key geometries in the SI. In order to reduce the computational costs and contain the geometric changes in the meaningful regions of the conformation space, a subset of the bond lengths, bond angles, and dihedral angles were chosen to be varied, while the others were constrained to the values at the minimum energy conformation. Since peripheral hydrogens should not have strong effects on the electronic structure and aromatic ring systems should be resistant to distortions, we chose the geometries of the single bonds that connect the aromatic ring systems as the variables. For a complete range of motion, six DOFs are required per bond: one bond length, two bond angles, and three dihedral angles. Hence, the number of variables in each of the model systems could be reduced to 30 or fewer Z-matrix coordinates. In order to assess the extent to which our choices of variables were justified, a constrained energy 
minimization was performed at the end of the TADF rate maximization, where we relaxed the bond lengths and angles that had been fixed in the TADF rate maximization.

\section{Results and Discussion}

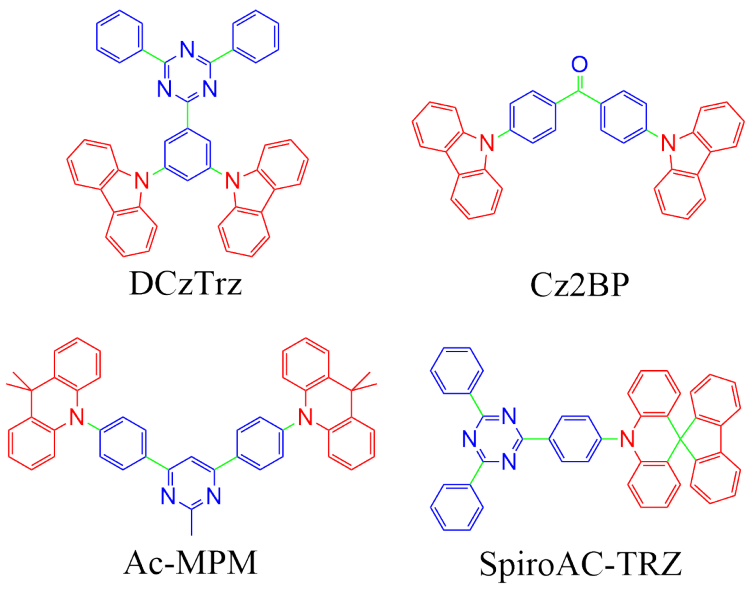

Figure 1: Skeletal structures of DCzTrz, Cz2BP, Ac-MPM, and SpiroAC-TRZ. The donor and acceptor groups are indicated in red and blue, respectively. Shown in green are the single bonds whose geometries were varied in the TADF rate maximization.

As model systems, we chose a number of TADF emitters that have been reported to exhibit high EQE in blue: DCzTrz, ${ }^{23}$ Cz2BP,${ }^{73}$ Ac-MPM, ${ }^{74}$ and SpiroAC-TRZ. ${ }^{25}$ The skeletal structures of the model systems are shown in Figure 1. We are going to examine the case of DCzTrz in detail and use the other emitters to assess the extent to which the results can be generalized.

\section{DCzTrz}

Table 1 summarizes the energetics of DCzTrz at the maximum TADF geometries with various energy penalties. Between the energy minimum $(\Lambda=\infty)$ and the TADF maximum with no energy penalty $(\Lambda=0)$, the TADF rate increases over two orders of magnitude from $9.4 \mathrm{~ms}^{-1}$ to $1.07 \mathrm{\mu s}^{-1}$. Moreover, the enhancement is not a result of trade-off between a small ST gap and a large TDM: the $\mathrm{S}_{1}-\mathrm{T}_{1}$ gap decreases from $0.13 \mathrm{eV}$ to $0.02 \mathrm{eV}$, and 
Table 1: Conformational energies, excitation energies, oscillator strengths, and TADF rates of DCzTrz at the maximum TADF geometries with various energy penalties. The case of $\Lambda=\infty$ corresponds to the energy minimum, and the case of $\Lambda=0$ corresponds to the TADF maximum with no energy penalty.

\begin{tabular}{|c|c|c|c|c|}
\hline $\begin{array}{c}\Lambda \\
\left({\mu \mathrm{s}^{-1} E_{\mathrm{h}}}^{-1}\right)\end{array}$ & $\begin{array}{c}\Delta E_{\mathrm{DFT}} \\
\left(\mathrm{kJ} \mathrm{mol}^{-1}\right)\end{array}$ & $\frac{\mathrm{EE}(\mathrm{eV})}{\mathrm{S}_{1} / \mathrm{T}_{1} / \mathrm{T}_{2}}$ & $f_{10}$ & $\begin{array}{l}k_{\mathrm{TADF}} \\
\left(\mu \mathrm{s}^{-1}\right)\end{array}$ \\
\hline$\infty$ & 0 & $2.84 / 2.71 / 2.74$ & 0.017 & 0.0094 \\
\hline 8.0 & 211 & $2.86 / 2.83 / 2.93$ & 0.026 & 0.88 \\
\hline 4.0 & 278 & $2.94 / 2.91 / 3.00$ & 0.029 & 1.06 \\
\hline 2.0 & 409 & $2.86 / 2.84 / 2.95$ & 0.026 & 1.08 \\
\hline 0.0 & 594 & $2.79 / 2.77 / 2.90$ & 0.028 & 1.07 \\
\hline
\end{tabular}

$f_{10}$ increases from 0.017 to 0.028 . The simultaneous improvement of what are supposed to be contraindicated properties confirms that conformational optimization has achieved more than modulation of the HOMO-LUMO overlap. Although the conformational energy also undergoes a gigantic increase of $594 \mathrm{~kJ} \mathrm{~mol}^{-1}$, the results with energy penalty reveal that much of the energy is irrelevant to improving the TADF rate. Using $\Lambda=4.0 \mathrm{\mu s}^{-1} E_{\mathrm{h}}{ }^{-1}$, the conformational energy can be halved with negligible loss in the TADF rate. Using $\Lambda=8.0 \mathrm{\mu s}^{-1} E_{\mathrm{h}}^{-1}$, further damping to $211 \mathrm{~kJ} \mathrm{~mol}^{-1}$ is possible with less than $20 \%$ loss of the TADF rate enhancement. The stability of the TADF rate against an energy penalty affirms that the maximum TADF conformation is not an absurdity, but its essential features are ones that one may hope to reproduce using suitable techniques in the real universe.

Figure 2a shows the minimum energy conformation with arrows indicating the DOFs that participate in the TADF rate maximization. It is obvious that the TADF maximum in Figure $2 \mathrm{~b}$ is a strained conformation. The carbazole $(\mathrm{Cz}$, donor) groups have popped above and below the plane of the 2,4,6-triphenyl-1,3,5-triazine (Trz, acceptor) group by $-33.4^{\circ}$ (left) and $+3.7^{\circ}$ (right), respectively. To be precise, two of the phenyl rings have twisted with respect to the triazine ring, so the Trz group is no longer planar, so these angles are with respect to the phenyl ring that bridges the $\mathrm{Cz}$ groups to the rest of the Trz group. Also, the $\mathrm{Cz}$ groups have rotated to become more orthogonal to the Trz group. The $\mathrm{Cz}$ groups are $92.7^{\circ}$ (left) and $79.8^{\circ}$ (right) to the Trz group, respectively, in contrast to $57.3^{\circ}$ (left) and 


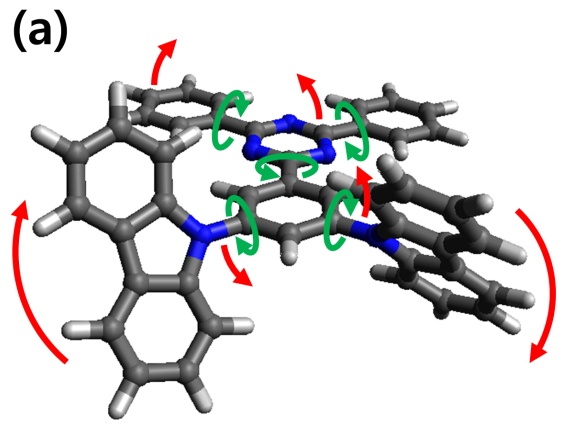

(d)

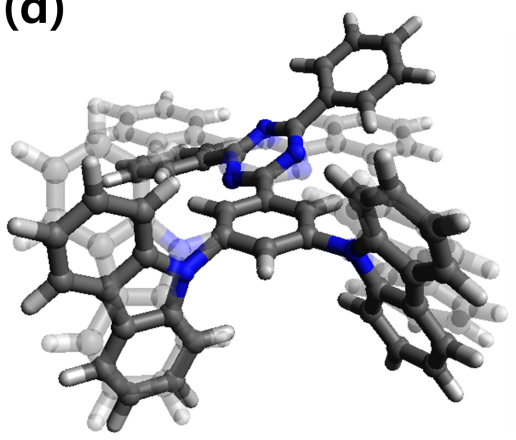

(b)

(e)

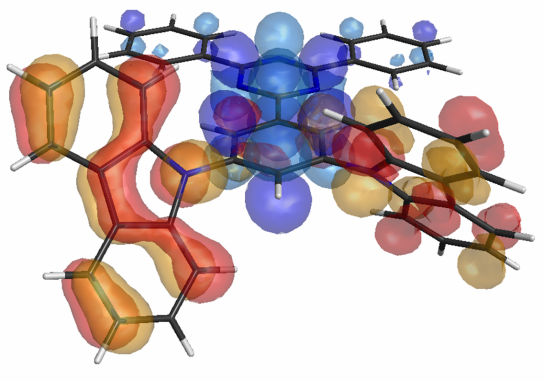

(c)

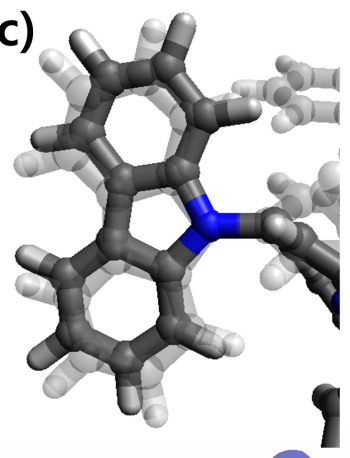

(f)

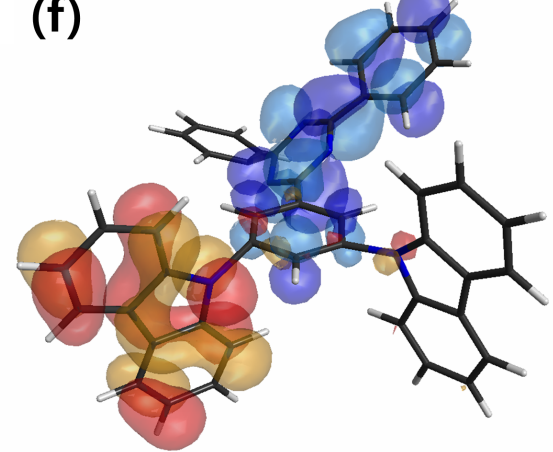

Figure 2: Geometries of DCzTrz at (a) the energy minimum $(\Lambda=\infty)$; (b) the TADF maximum with no energy penalty $(\Lambda=0)$; (c) the donor-acceptor bond (maginfied view); and (d) the TADF maximum with energy penalty $\left(\Lambda=8.0 \mathrm{\mu s}^{-1} E_{\mathrm{h}}{ }^{-1}\right)$. Also, the dominant NTO pairs of the $S_{1}$ state at (e) the energy minimum $(\Lambda=\infty)$ and (f) the TADF maximum with no energy penalty $(\Lambda=0)$. The arrows in (a) indicate the DOFs that are the most active in the TADF rate maximization, and the translucent geometries in the background of (b-d) are the minimum energy conformation. In (e-f), the hole orbitals are shown in red and orange, and the electron orbitals are shown in sky blue and indigo. Contour value: 0.025 . 
$56.5^{\circ}$ (right) at the energy minimum. Increasing the dihedral angle between the donor and acceptor moieties is a known strategy to reduce the HOMO-LUMO overlap and hence the ST gap. ${ }^{75-77}$ The fact that the same motif appears at the TADF maximum of DCzTrz is an encouraging sign that useful design principles can be extracted.

Indeed, a strange feature comes to attention. After popping above and below the plane of the Trz group, the $\mathrm{Cz}$ groups tilt back towards the Trz group, as shown in Figure 2c. The lower and upper $\mathrm{C}_{\mathrm{Cz}}-\mathrm{N}_{\mathrm{Cz}}-\mathrm{C}_{\mathrm{Trz}}$ angles at each $\mathrm{Cz}$ group differ by $+17.7^{\circ}$ (left) and $-19.5^{\circ}$ (right), respectively, whereas the $\mathrm{C}_{\mathrm{Cz}}-\mathrm{N}_{\mathrm{Cz}}-\mathrm{C}_{\mathrm{Trz}}$ angles at the energy minimum are the same $125.7^{\circ} \pm 0.2^{\circ}$. Though the differences in the angles change to $+23.8^{\circ}$ (left) and $-6.5^{\circ}$ (right), the gooseneck persists even with an energy penalty of $\Lambda=8.0 \mathrm{\mu s}^{-1} E_{\mathrm{h}}{ }^{-1}$. Hence, the gooseneck cannot be a side product of the optimization, but a feature relevant to TADF. Though the physical intuition is unclear, it might be associated with the additional terms in the four-state model ${ }^{31}$ or the dissimilar forms of the integrands in the exchange integral $\left\langle r_{12}{ }^{-1}\right\rangle$ and the transition dipole integral $\left\langle r_{1}\right\rangle$. After all, the ST gap and the TDM should depend not only on the overlap, but also on the orientation of the HOMO and the LUMO.

Meanwhile, the twist in the Trz group tends to dissipate with energy penalty. As shown in Figure 2d, the phenyl rings return to a more coplanar arrangement to the triazine ring in what appears to be a concerted manner. However, the planarity of the Trz group remains quite disturbed even with an energy penalty of $\Lambda=8.0 \mathrm{\mu s}^{-1} E_{\mathrm{h}}{ }^{-1}$. The phenyl rings to the front center and the back left are $30.2^{\circ}$ and $30.6^{\circ}$ to the triazine ring, respectively, which are $41.6^{\circ}$ and $61.3^{\circ}$ in the absence of energy penalty. We conjecture that the twist in the Trz group controls the distribution of the LUMO so that it gets neither too close to nor too far away from to the HOMO. Comparing the dominant NTO pairs of the $\mathrm{S}_{1}$ state at the energy minimum (Figure 2e) and the TADF maximum (Figure 2f), the distribution of the $\mathrm{S}_{1}$ hole is indeed impacted. The NTOs of the $\mathrm{T}_{1}$ state are similar (Figure $\mathrm{S} 4$ ). This would also explains why the twist appears to unroll in a concerted manner as energy penalties are 
imposed. Overall, the twist in the Trz group appears to help enhance the TADF rate, but a balance must be struck with the conformational energy.

\section{Other Model Systems}

Table 2: Conformational energies, excitation energies, oscillator strengths, and TADF rates of Cz2BP, Ac-MPM, and SpiroAC-TRZ at the maximum TADF geometries with various energy penalties. The case of $\Lambda=\infty$ corresponds to the energy minimum, and the case of $\Lambda=0$ corresponds to the TADF maximum with no energy penalty.

\begin{tabular}{|c|c|c|c|c|c|}
\hline & $\begin{array}{c}\Lambda \\
\left({\left.\mu \mathrm{s}^{-1} E_{\mathrm{h}}{ }^{-1}\right)}^{-1}\right.\end{array}$ & $\begin{array}{c}\Delta E_{\mathrm{DFT}} \\
\left(\mathrm{kJ} \mathrm{mol}^{-1}\right)\end{array}$ & $\frac{\mathrm{EE}(\mathrm{eV})}{\mathrm{S}_{1} / \mathrm{T}_{1} / \mathrm{T}_{2}}$ & $f_{10}$ & $\begin{array}{l}k_{\mathrm{TADF}} \\
\left(\mu \mathrm{s}^{-1}\right)\end{array}$ \\
\hline \multirow{4}{*}{ 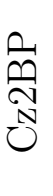 } & inf & 0 & $2.99 / 2.67 / 2.78$ & 0.345 & $1.9 \times 10^{-4}$ \\
\hline & 8.0 & 162 & $2.67 / 2.63 / 2.64$ & 0.061 & 0.66 \\
\hline & 4.0 & 181 & $2.66 / 2.62 / 2.62$ & 0.062 & 0.69 \\
\hline & 0.0 & 193 & $2.64 / 2.60 / 2.61$ & 0.060 & 0.69 \\
\hline \multirow{4}{*}{ 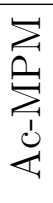 } & inf & 0 & $2.47 / 2.46 / 2.47$ & $3.4 \times 10^{-5}$ & 0.0012 \\
\hline & 8.0 & 51 & $2.51 / 2.49 / 2.49$ & 0.036 & 0.59 \\
\hline & 4.0 & 365 & $2.54 / 2.51 / 2.67$ & 0.022 & 0.58 \\
\hline & 0.0 & 420 & $2.55 / 2.52 / 2.61$ & 0.028 & 0.63 \\
\hline \multirow{4}{*}{ 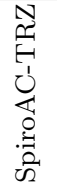 } & inf & 0 & $2.41 / 2.41 / 2.66$ & $7.9 \times 10^{-6}$ & $4.2 \times 10^{-4}$ \\
\hline & 8.0 & 104 & $2.56 / 2.54 / 2.75$ & 0.012 & 0.49 \\
\hline & 4.0 & 294 & $2.48 / 2.46 / 2.75$ & 0.017 & 0.50 \\
\hline & 0.0 & 475 & $2.50 / 2.48 / 2.67$ & 0.019 & 0.61 \\
\hline
\end{tabular}

Table 2 summarizes the energetics of Cz2BP, Ac-MPM, and SpiroAC-TRZ. The TADF rates start in the millisecond timescale at the energy minimum and end in the microsecond timescale at the TADF maximum, suggesting that TADF rate enhancements over two to three orders of magnitude might be typical in the conformational optimization of TADF emitters. Cz2BP undergoes the largest enhancement in the TADF rate, by a factor of 3000. However, the improvement is a result of trade-off between a small ST gap and a large TDM: the $\mathrm{S}_{1}-\mathrm{T}_{1}$ gap and $f_{10}$ both decrease from $0.32 \mathrm{eV}$ to $0.04 \mathrm{eV}$ and from 0.345 to 0.060, respectively. The cases of Ac-MPM and SpiroAC-TRZ might be more complicated. Whereas the increases in the $\mathrm{S}_{1}-\mathrm{T}_{1}$ gaps of $0.02 \mathrm{eV}$ are comparable to the uncertainty, the increases in the $f_{10}$ of Ac-MPM and SpiroAC-TRZ span three orders of magnitude from $3.4 \times 10^{-5}$ to 0.028 and from $7.9 \times 10^{-6}$ to 0.019 , respectively. The disproportion sug- 
gests that conformational optimization might have had a non-trivial impact on the electronic structure. In any case, simultaneous improvement of the ST gap and the TDM might be a rare occurance, which might also explain why DCzTrz was the only model system whose TADF rate reached the submicrosecond regime.

Notice that the minimum energy conformations of Ac-MPM and SpiroAC-TRZ have a tiny ST gap. In particular, the ST gap of SpiroAC-TRZ is near vanishing. The two-state model would suggest that the SOC must vanish, since both the $\mathrm{S}_{1}$ and $\mathrm{T}_{1}$ states must be CT states. ${ }^{27}$ Surprisingly, the RISC step does not appear to be the most severe bottleneck. The $f_{10}$ and SOC are $7.9 \times 10^{-6}$ and $0.0063 \mathrm{~cm}^{-1}$, respectively, which correspond to a fluorescence rate of $2.0 \mathrm{~ms}^{-1}$ and a RISC rate of $2.9 \mathrm{~ms}^{-1}$ (Table S1). This makes sense in light of the four-state model. The $\mathrm{S}_{1}$ and $\mathrm{T}_{1}$ states contain different fractions of the diabatic CT and LE states, and the electron exchange favors the $\mathrm{S}_{1}$ state as the more CT-like state. Hence, the fluorescence step can end up slower than the RISC step. We refer interested readers to the work of de Silva et al. ${ }^{31}$

In each of the model systems, a suitable energy penalty can reduce the conformational energy with minimal loss in the TADF rate. Even with no energy penalty, the TADF maximum of Cz2BP has a conformational energy of $194 \mathrm{~kJ} \mathrm{~mol}^{-1}$, less than half of the other model systems. With an energy penalty of $\Lambda=8.0 \mathrm{\mu s}^{-1} E_{\mathrm{h}}{ }^{-1}$, Ac-MPM and SpiroAC-TRZ have conformational energies of $53 \mathrm{~kJ} \mathrm{~mol}^{-1}$ and $102 \mathrm{~kJ} \mathrm{~mol}^{-1}$, respectively, while retaining more than $90 \%$ and $80 \%$ of the TADF rates at the maximum with no energy penalty. Though these conformational energies might seem daunting, synthetic chemists have created and stabilized molecules with strain energies over $300 \mathrm{~kJ} \mathrm{~mol}^{-1} \cdot{ }^{78,79}$ For certain TADF emitters, the maximum TADF conformation might be accessible using suitable techniques in the real universe.

Figure 3 shows the geometries of $\mathbf{C z 2 B P}$, Ac-MPM, and SpiroAC-TRZ at the TADF maximum with no energy penalty $(\Lambda=0)$. The maximum TADF geometries with energy penalties are available in the SI. A number of patterns can be noted. First, the dihedral 
(a)

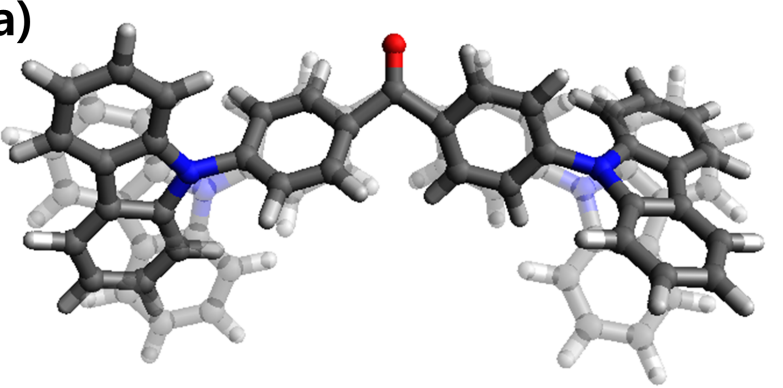

(c)

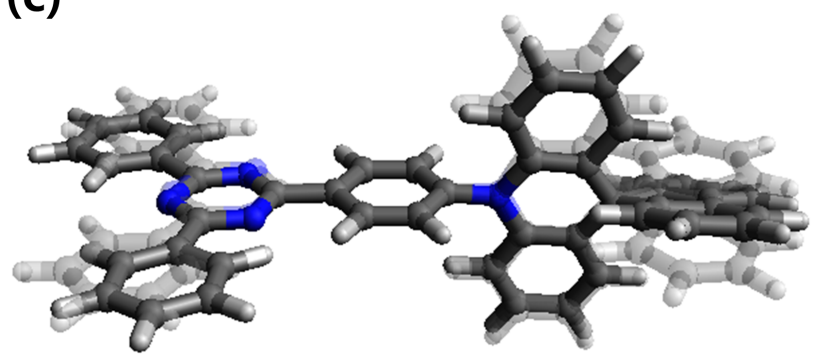

(b)

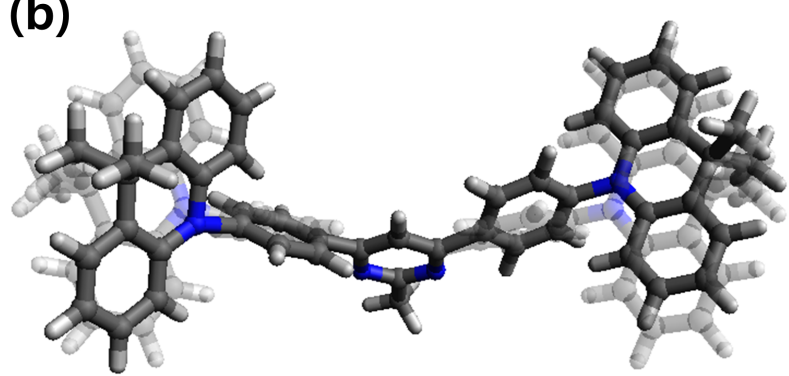

(d)

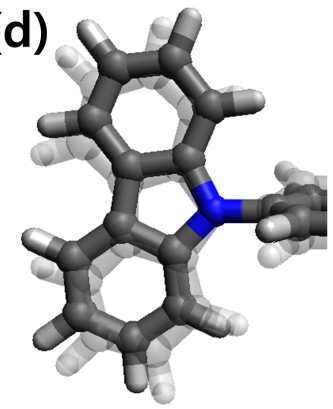

(e)

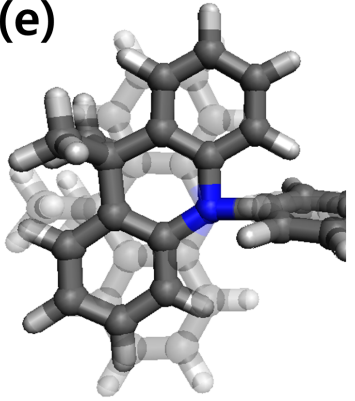

Figure 3: Geometries of (a) Cz2BP; (b) Ac-MPM; and (c) SprioAC-TRZ at the TADF maximum with no energy penalty $(\Lambda=0)$. Also, magnified views of the donor-acceptor bonds in (d) Cz2BP and (e) Ac-MPM. The translucent geometries in the background are the minimum energy conformations.

angles between the donor and acceptor groups increase in Cz2BP (Figure 3a), while they decrease in Ac-MPM (Figure 3b) and SpiroAC-TRZ (Figure 3c). The dihedral angle changes are consistent with the outcome that the ST gap and the TDM both decrease in Cz2BP and increase in Ac-MPM and SpiroAC-TRZ. Second, SpiroAC-TRZ is the only model system that does not develop a gooseneck. The donor groups of Cz2BP (Figure 3d) and Ac-MPM (Figure 3e) both pop above and below the plane of the acceptor group and then tilt back towards the acceptor group. Third, the acceptor groups of $\mathbf{C z 2 B P}$ and Ac-MPM become twisted, albeit in different ways from both DCzTrz and each other. In Cz2BP, the phenyl rings become more orthogonal to each other. In Ac-MPM, one of the phenyl rings twists out of plane, while the other maintains a moderate angle to the pyrimidine ring. The gooseneck and the twist in the acceptor group tend to persist even with energy penalties.

Remarkably, three out of the four model systems developed a gooseneck at the DA bond and a twist in the acceptor group. We suspect that these motifs might provide a useful 
handle on the TADF rate in general DA systems. Unfortunately, there is want of theoretical intuition and experimental evidence at this point. Inspite of abundant efforts to manipulate the DA dihderal angles, ${ }^{75-77}$ we are not aware of prior studies that have considered outof-plane displacement of the donor and the acceptor moieties. As discussed earlier, the gooseneck might be associated with the mathematical forms of the integrals that enter the TADF rate, but it is unclear which integrals are affected in what way.

The twist in the acceptor group is also obscure. While it is not hard to rationalize how the twist might change the distribution of the LUMO, it is not always clear why a particular distribution should be more conducive to TADF than another, nor does there appear to be a pattern in how the acceptor groups become distorted. For example, Ac-MPM appears to push the LUMO towards just one of the donor groups, while $\mathbf{C z 2 B P}$ remains much more symmetric. The dominant NTO pairs of the $\mathrm{S}_{1}$ and $\mathrm{T}_{1}$ states can be found in the SI. Perhaps, the absence of a trend is due to the small number of model systems. The twist might not be a single motif, but a collection of motifs that concern specific families of emitters.

At the same time, the lack of physical intuition attests to the true value of conformational optimization: discovery of design principles that do not render themselves to deduction.

\section{Choice of Density Functional}

In order to assess the functional dependence of the results, we took the maximum TADF geometries optimized using B3LYP and recalculated the electronic structures using PBE0, M06-2X, and LRC- $\omega^{*} \mathrm{PBE}$. To our surprise, the generalized gradient approximation (GGA) global hybrid, the meta-GGA global hybrid, and the range-separated hybrid (RSH) exhibited similar patterns. Since tuned RSHs have been shown to be effective at predicting the excited state properties of TADF emitters, ${ }^{40,80}$ we focus on LRC- $\omega^{*} \mathrm{PBE}$ as the primary subject of comparison. The complete set of results can be found in the SI.

Table 3 summarizes the LRC- $\omega^{*}$ PBE energetics of DCzTrz at the B3LYP geometries. Interestingly, the TADF rates in $\mathrm{LRC}-\omega^{*} \mathrm{PBE}$ seem to increase with the energy penalty in 
Table 3: Conformational energies, excitation energies, oscillator strengths, and TADF rates of DCzTrz recomputed using LRC- $\omega^{*} \mathrm{PBE} / \mathrm{IEF}-\mathrm{PCM}$ at the maximum TADF geometries optimized using B3LYP. The case of $\Lambda=\infty$ corresponds to the energy minimum, and the case of $\Lambda=0$ corresponds to the TADF maximum with no energy penalty.

\begin{tabular}{|c|c|c|c|c|c|}
\hline Solvent & $\begin{array}{c}\Lambda \\
\left(\mathrm{us}^{-1} E_{\mathrm{h}}{ }^{-1}\right)\end{array}$ & $\begin{array}{c}\Delta E_{\mathrm{DFT}} \\
\left(\mathrm{kJ} \mathrm{mol}^{-1}\right)\end{array}$ & $\frac{\mathrm{EE}(\mathrm{eV})}{\mathrm{S}_{1} / \mathrm{T}_{1} / \mathrm{T}_{2}}$ & $f_{10}$ & $\begin{array}{l}k_{\mathrm{TADF}} \\
\left(\mu \mathrm{s}^{-1}\right)\end{array}$ \\
\hline \multirow{5}{*}{ vacuum } & inf & 0 & $3.39 / 3.07 / 3.14$ & 0.0310 & $1.5 \times 10^{-5}$ \\
\hline & 8.0 & 202 & 3.49 / $3.27 / 3.29$ & 0.0133 & $2.8 \times 10^{-4}$ \\
\hline & 4.0 & 267 & 3.49 / 3.26 / 3.27 & 0.0018 & $2.4 \times 10^{-5}$ \\
\hline & 2.0 & 394 & $3.37 / 3.15 / 3.20$ & $3.5 \times 10^{-4}$ & $7.1 \times 10^{-6}$ \\
\hline & 0.0 & 573 & $3.33 / 2.93 / 3.11$ & $3.9 \times 10^{-4}$ & $1.1 \times 10^{-8}$ \\
\hline \multirow{5}{*}{ toluene } & inf & 0 & $3.30 / 3.07 / 3.08$ & 0.0312 & $3.1 \times 10^{-4}$ \\
\hline & 8.0 & 199 & $3.37 / 3.27 / 3.27$ & 0.0146 & 0.024 \\
\hline & 4.0 & 262 & 3.39 / $3.27 / 3.27$ & 0.0021 & 0.0019 \\
\hline & 2.0 & 389 & $3.28 / 3.14 / 3.21$ & $4.2 \times 10^{-4}$ & $2.5 \times 10^{-4}$ \\
\hline & 0.0 & 568 & $3.24 / 2.93 / 3.12$ & $3.4 \times 10^{-4}$ & $2.7 \times 10^{-7}$ \\
\hline
\end{tabular}

B3LYP. The TADF rate at the TADF maximum with no energy penalty reduces to $0.011 \mathrm{~s}^{-1}$, which is smaller than $15 \mathrm{~s}^{-1}$ at the energy minimum. On the other hand, the TADF rate at the geometry optimized with $\Lambda=8.0 \mathrm{us}^{-1} E_{\mathrm{h}}^{-1}$ is $280 \mathrm{~s}^{-1}$, an order of magnitude greater than that at the energy minimum. A toluene-like environment stabilizes the $\mathrm{S}_{1}$ state by $0.1 \mathrm{eV}$ to $0.2 \mathrm{eV}$, increasing the maximum TADF rate with energy penalty to $24 \mathrm{~ms}^{-1}$ and also the enhancement factor to two orders of magnitude greater than $0.31 \mathrm{~ms}^{-1}$ at the energy minimum. The other molecules exhibit similar trends, as shown in Tables S4 and S6. We suspect that the TADF rate maximization had a propensity to exploit the quirks of the B3LYP universe and that the energy penalty helped the maximization to remain in the physical region of the conformation space. The existence of such quirks is not surprising, since B3LYP is just an approximation to the true density functional. In fact, we might have encountered the same issue even if we had optimized the geometries using another functional and recalculated the electronic structures using B3LYP.

In any case, there are noteworthy commonalities in the energetics predicted by B3LYP and the other functionals. First, there exists a conformation where the TADF rate is orders of magnitude greater than at the minimum energy conformation. Moreover, the maximum 
TADF conformation in B3LYP and those in the other functionals cannot be too far apart, considering that a moderate energy penalty is sufficient to arrive at a geometry that exhibits TADF rate enhancement in all of the functionals. These observations reaffirm that the maximum TADF conformation not only exists, but also can be determined to a reasonable precision.

Second, one or more of the model systems exhibit a simultaneous improvement of the ST gap and TDM. These are DCzTrz in B3LYP and PBE0 and Ac-MPM and SpiroACTRZ in M06-2X/IEF-PCM and LRC- $\omega^{*}$ PBE/IEF-PCM. When a simultaneous improvement of the ST gap and TDM occurs, the maximium TADF rate can be impressive. In B3LYP, the TADF rate of DCzTrz reaches the submicrosecond timescales. In LRC- $\omega^{*} \mathrm{PBE} / \mathrm{IEF}-$

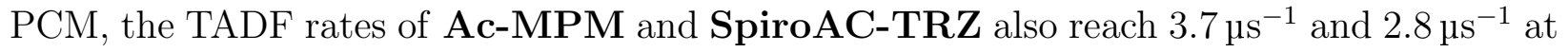
their respective TADF maxima with $\Lambda=8.0 \mathrm{\mu s}^{-1} E_{\mathrm{h}}{ }^{-1}$ (Table S6). Indeed, it is concerning that different functionals predict simultaneous improvement in different molecules, and it is possible that the simultaneous improvement is just a quirk in each of the functionals. Nonetheless, the results cast a serious doubt on the conventional wisdom that the trade-off is an inherent property of TADF emitters.

\section{Choice of Conformational Variables}

So far, we have assumed that the variables in the TADF rate maximization corresponded to the most flexible DOFs in the model systems. While the assumption does not affect the existence of conformations that maximize the TADF rate, it does affect the extent to which we can expect the maximum TADF conformation to be reproducible in the real universe. If our choice of conformational variables were appropriate, then the changes in the geometry and the consequent changes in the TADF rate would be minimal even if we relaxed the complementary DOFs. Hence, we took the maximum TADF geometries and performed a constrained energy minimization to relax the hydrogen atoms and the aromatic ring systems, which had been fixed in the TADF rate maximization. 

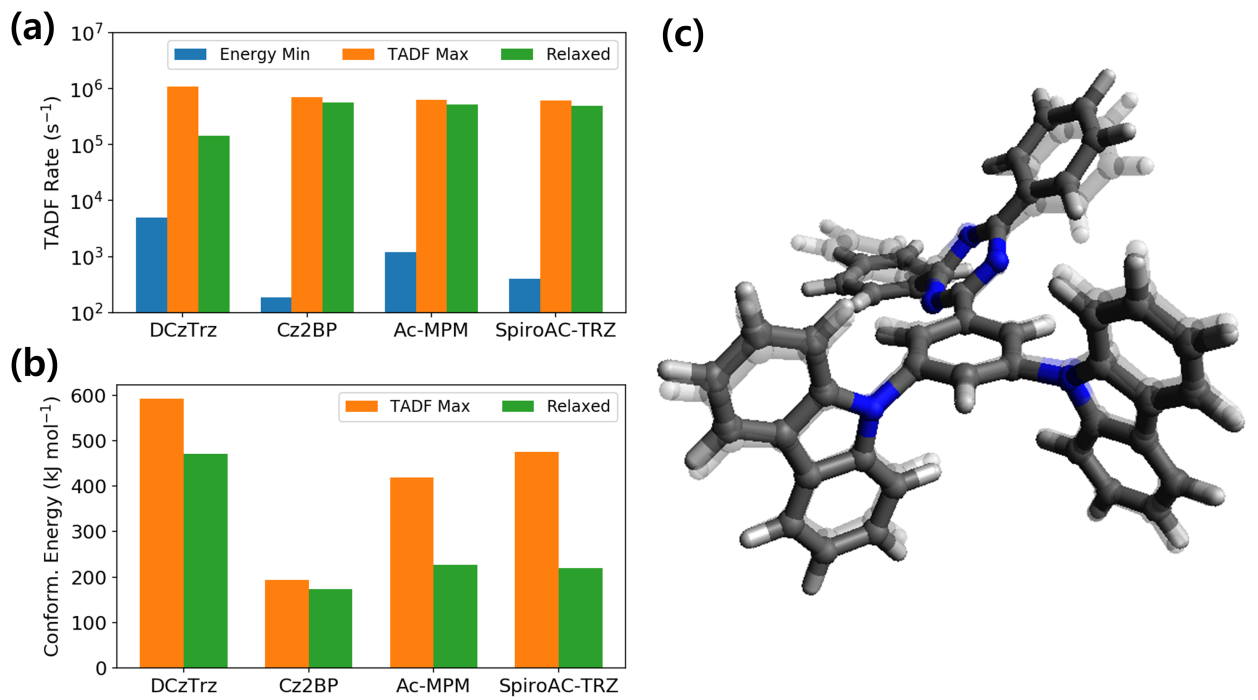

Figure 4: (a) TADF rates and (b) DFT energies of DCzTrz, Cz2BP, Ac-MPM, and SprioAC-TRZ at the energy minimum, at the TADF maximum $(\Lambda=0)$, and after the relaxation of the hydrogen atoms and the aromatic ring systems. (c) Geometry of DCzTrz at the TADF maximum $(\Lambda=0)$ after the relaxation of the hydrogen atoms and the aromatic ring systems.

Figure 4 summarizes the energetics of the maximum TADF geometries before and after the constrained energy minimization. The effects of the relaxation depend on the system. For example, DCzTrz undergoes an order-of-magnitude decrease in the TADF rate from $1.07 \mathrm{\mu s}^{-1}$ to $0.14 \mathrm{\mu s}^{-1}$, and a noticeable decrease in the DFT energy from $594 \mathrm{~kJ} \mathrm{~mol}^{-1}$ to $472 \mathrm{~kJ} \mathrm{~mol}^{-1}$, as shown in Figures $4 \mathrm{a}$ and Figure 4b, respectively. It appears that our choice of variables included too many rigid DOFs, or the geometric changes might have been severe enough to obscure the distinction of flexible and rigid DOFs. Figure 2c reveals that the constrained energy minimization causes distortions in the phenyl and the triazine rings that tend to restore the planarity of the Trz group. These changes agree with the conjecture that the twist in the acceptor group imparts a considerable strain on DCzTrz. Also, it makes sense that unrolling the twist in the acceptor group in an unconcerted manner would be detrimental to the TADF rate, since the distortion controls the distribution of the LUMO.

With the exception of DCzTrz, the TADF rate enhancement is quite stable against the relaxation of the hydrogen atoms and the aromatic ring systems. Cz2BP shows minimal 
changes in both the TADF rate and the DFT energy, meaning that our choice of variables captured both the most flexible DOFs and the DOFs that are the most relevant to the TADF rate. In the cases of Ac-MPM and SprioAC-TRZ, the conformational energies are halved, but the TADF rates remain at $0.52 \mu^{-1}$ and $0.49 \mu^{-1}$, respectively. The geometries of Cz2BP, Ac-MPM, and SprioAC-TRZ after the constrained energy minimization have been provided in the SI. In addition to affirming our choice of variables, the stability of the TADF rate suggests a substantial overlap between the most flexible DOFs and the DOFs that determine the TADF rate in the model systems. The outcome is consistent with our proposition that the essential features of the maximum TADF conformation might be reproducible using suitable techniques in the real universe.

How to synthesize the maximum TADF conformers is beyond the scope of this work, but we can suggest some basic examples. The textbook chemist's approach would be to attach bulky groups that constrain the rotation of certain bonds - the same approach has been used to modulate the donor-acceptor dihedral angles in TADF emitters. ${ }^{75-77}$ We can also imagine placing the organic layer under mechanical stress or pressure to crowd the molecules together. In the first place, it is not necessary to have every OLED molecule in the maximum TADF conformation. For most practical purposes, it would suffice to increase the population of molecules that have higher TADF rate than the equilibrium ensemble of the original emitter. We expect that a myriad of techniques in enzyme design might prove applicable to the conformational engineering of TADF OLEDs.

\section{Conclusions}

In order to demonstrate the potential of conformational optimization to assist the development of trend-breaking TADF OLEDs, we studied direct maximization of the TADF rate in the conformation spaces of several TADF emitters. As desired, one of the model systems (DCzTrz) achieved a simultaneous improvement of the ST gap and the TDM, and its simu- 
lated TADF rate could be brought into the submicrosecond timescales. Even model systems that exhibited trade-off between a small ST gap and a large TDM were found to undergo TADF rate enhancements by two to three orders of magnitude. The conformational energy required to maximize the $\mathrm{TADF}$ rate can be as small as $193 \mathrm{~kJ} \mathrm{~mol}^{-1}$ even in the absence of energy penalty (Cz2BP), and the conformational energy can be as small as $51 \mathrm{~kJ} \mathrm{~mol}^{-1}$ with minimal loss in the TADF rate enhancement (Ac-MPM). Furthermore, the maximum TADF conformations of the model systems shared a number of structural motifs, such as the gooseneck at the DA bond and the concerted rotation of the aromatic rings in the acceptor group. Although the physical intuition behind these motifs remain obscure, the opacity itself demonstrates the true value of conformational optimization: discovery of design principles that do not render themselves to deduction.

The conformational dependence of the TADF rate motivates the incorporation of con-

formational engineering into the TADF technology. Based on the results of TADF rate maximization with energy penalties and constrained energy minimization, we propose that the maximum TADF conformation of certain molecules, or another conformation that contains its essential features, might be accessible under high levels of strain that are within the reach of modern synthetic chemistry. Since the minimum energy conformation is not the best geometry in terms of the TADF rate, it would be desirable to manipulate the conformation in a way that enhances the TADF rate. Although the proposition might seem unorthodox in physical or organic chemistry, changing the properties of a molecule by changing its conformation is a standard in fields such as enzyme design. In the future, we hope to connect conformational optimization with experimental efforts and demonstrate that the electronic structures of TADF emitters can be improved in the real universe.

\section{Acknowledgement}

This work was supported by a grant from the U.S. Department of Energy Office of Basic 
Energy Sciences (DE-FG02-07ER46474).

\section{Supporting Information Available}

Our version of the Nelder-Mead simplex method; calculation of the RISC rate and evaluation of its effects on the TADF rate; recalculation of the electronic structures using PBE0, M062X, LRC- $\omega^{*}$ PBE, M06-2X/IEF-PCM and LRC- $\omega^{*}$ PBE/IEF-PCM; geometries of Cz2BP, Ac-MPM, and SprioAC-TRZ at the TADF maximum with various energy penalties, and after the relaxation of DOFs that had been fixed in the TADF rate maximization; dominant NTO pairs of DCzTrz, Cz2BP, Ac-MPM, and SprioAC-TRZ in the $\mathrm{S}_{1}$ and $\mathrm{T}_{1}$ states; Z-matrices thereof.

\section{References}

(1) Tang, C. W.; VanSlyke, S. A. Organic electroluminescent diodes. Appl. Phys. Lett. 1987, 51, 913-915.

(2) Burrows, P. E.; Gu, G.; Bulovic, V.; Shen, Z.; Forrest, S. R.; Thompson, M. E. Achieving full-color organic light-emitting devices for lightweight, flat-panel displays. IEEE Trans. Electron Devices 1997, 44, 1188-1203.

(3) Geffroy, B.; le Roy, P.; Prat, C. Organic light-emitting diode (OLED) technology: materials, devices and display technologies. Polym. Int. 2006, 55, 572-582.

(4) Xiao, L.; Chen, Z.; Qu, B.; Luo, J.; Kong, S.; Gong, Q.; Kido, J. Recent Progresses on Materials for Electrophosphorescent Organic Light-Emitting Devices. Adv. Mater. 2011, 23, 926-952.

(5) de Almeida, A.; Zissis, G.; Quicheron, M.; Bertldi, P. Accelerating the Deployment of 
Solid State Lighting (SSL) in Europe; EUR 25596 EN; European Commission Joint Research Centre: Ispra (VA), Italy, 2012.

(6) Penning, J.; Stober, K.; Taylor, V.; Yamada, M. Energy Savings Forecast of SolidState Lighting in General Illumination Applications; DOE/EE-1467; U.S. Department of Energy Office of Energy Efficiency and Renewable Energy: Washington D.C., 2016.

(7) Sasabe, H.; Kido, J. Recent Progress in Phosphorescent Organic Light-Emitting Devices. Eur. J. Org. Chem. 2013, 7653-7663.

(8) Wong, M. Y.; Zysman-Colman, E. Purely Organic Thermally Activated Delayed Fluorescence Materials for Organic LightEmitting Diodes. Adv. Mater. 2017, 29, 1605444.

(9) Baldo, M. A.; O’Brien, D. F.; You, Y.; Shoustikov, A.; Sibley, S.; Thompson, M. E.; Forrest, S. R. Highly efficient phosphorescent emission from organic electroluminescent devices. Nature 1998, 395, 151-154.

(10) Minaev, B.; Baryshnikov, G.; Agren, H. Principles of phosphorescent organic light emitting devices. Phys. Chem. Chem. Phys. 2014, 16, 1719-1758.

(11) Zhang, Y.; Lee, J.; Forrest, S. R. Tenfold increase in the lifetime of blue phosphorescent organic light-emitting diodes. Nat. Commun. 2014, 5, 1-7.

(12) Parker, C. A.; Hatchard, C. G. Triplet-singlet emission in fluid solutions. Phosphorescence of eosin. Trans. Faraday Soc. 1961, 57, 1894-1904.

(13) Endo, A.; Ogasawara, M.; Takahashi, A.; Yokoyama, D.; Kato, Y.; Adachi, C. Thermally Activated Delayed Fluorescence from $\mathrm{Sn}^{4+}-$ Porphyrin Complexes and Their Application to Organic Light Emitting Diodes - A Novel Mechanism for Electroluminescence. Adv. Mater. 2009, 21, 4802-4806.

(14) Endo, A.; Sato, K.; Yoshimura, K.; Kai, T.; Kawada, A.; Miyazaki, H.; Adachi, C. 
Efficient up-conversion of triplet excitons into a singlet state and its application for organic light emitting diodes. Appl. Phys. Lett. 2011, 98, 083302.

(15) Uoyama, H.; Goushi, K.; Shizu, K.; Nomura, H.; Adachi, C. Highly efficient organic light-emitting diodes from delayed fluorescence. Nature 2012, 492, 234-238.

(16) Lee, J.; Shizu, K.; Tanaka, H.; Nomura, H.; Yasuda, T.; Adachi, C. Oxadiazole-and triazole-based highly-efficient thermally activated delayed fluorescence emitters for organic light-emitting diodes. J. Mater. Chem. C 2013, 1, 4599-4604.

(17) Kawasumi, K.; Wu, T.; Zhu, T.; Chae, H. S.; Van Voorhis, T.; Baldo, M. A.; Swager, T. M. Thermally Activated Delayed Fluorescence Materials Based on Homoconjugation Effect of Donor-Acceptor Triptycenes. J. Am. Chem. Soc. 2015, 137, 1190811911.

(18) Liu, Y.; Xie, G.; Wu, K.; Luo, Z.; Zhou, T.; Zeng, X.; Yu, J.; Gong, S.; Yang, C. Boosting reverse intersystem crossing by increasing donors in triarylboron/phenoxazine hybrids: TADF emitters for high-performance solution-processed OLEDs. J. Mater. Chem. C 2016, 4, 4402-4407.

(19) Nobuyasu, R. S.; Ren, Z.; Griffiths, G. C.; Batsanov, A. S.; Data, P.; Yan, S.; Monkman, A. P.; Bryce, M. R.; Dias, F. B. Rational Design of TADF Polymers Using a Donor-Acceptor Monomer with Enhanced TADF Efficiency Induced by the Energy Alignment of Charge Transfer and Local Triplet Excited States. Adv. Opt. Mater. 2016, 4, 597-607.

(20) Sarma, M.; Wong, K.-T. Exciplex: An Intermolecular Charge-Transfer Approach for TADF. ACS Appl. Mater. Interfaces 2018, 10, 19279-19304.

(21) Shu, Y.; Levine, B. G. Simulated evolution of fluorophores for light emitting diodes. J. Chem. Phys. 2015, 142, 104104. 
(22) Gómez-Bombarelli, R.; Aguilera-Iparraguirre, J.; Hirzel, T. D.; Duvenaud, D.; Maclaurin, D.; Blood-Forsythe, M. A.; Chae, H. S.; Einzinger, M.; Ha, D.-G.; Wu, T. et al. Design of efficient molecular organic light-emitting diodes by a high-throughput virtual screening and experimental approach. Nat. Mater. 2016, 15, 1120-1127.

(23) Kim, M.; Jeon, S. K.; Hwang, S.-H.; Lee, J. Y. Stable Blue Thermally Activated Delayed Fluorescent Organic LightEmitting Diodes with Three Times Longer Lifetime than Phosphorescent Organic LightEmitting Diodes. Adv. Mater. 2015, 27, 2515-2520.

(24) dos Santos, P. L.; Ward, J. S.; Bryce, M. R.; Monkman, A. P. Using Guest-Host Interactions To Optimize the Efficiency of TADF OLEDs. J. Phys. Chem. Lett. 2016, 7, 3341-3346.

(25) Lin, T.-A.; Chatterjee, T.; Tsai, W.-L.; Lee, W.-K.; Wu, M.-J.; Jiao, M.; Pan, K.-C.; Yi, C.-L.; Chung, C.-L.; Wong, K.-T. et al. Sky-Blue Organic Light Emitting Diode with 37\% External Quantum Efficiency Using Thermally Activated Delayed Fluorescence from Spiroacridine-Triazine Hybrid. Adv. Mater. 2016, 28, 6976-6983.

(26) Cui, L.-S.; Nomura, H.; Geng, Y.; Kim, J. U.; Nakanotani, H.; Adachi, C. Controlling Singlet-Triplet Energy Splitting for Deep-Blue Thermally Activated Delayed Fluorescence Emitters. Angew. Chem. Int. Ed. 2017, 56, 1571-1575.

(27) El-Sayed, M. SpinOrbit Coupling and the Radiationless Processes in Nitrogen Heterocyclics. J. Chem. Phys. 1963, 38, 2834-2838.

(28) Etherington, M. K.; Gibson, J.; Higginbotham, H. F.; Penfold, T. J.; Monkman, A. P. Revealing the spin-vibronic coupling mechanism of thermally activated delayed fluorescence. Nat. Commun. 2016, 7, 13680.

(29) Gibson, J.; Monkman, A. P.; Penfold, T. J. The Importance of Vibronic Coupling for Efficient Reverse Intersystem Crossing in Thermally Activated Delayed Fluorescence Molecules. ChemPhysChem 2016, 17, 2956-2961. 
(30) Samanta, P. K.; Kim, D.; Coropceanu, V.; Brédas, J.-L. Up-Conversion Intersystem Crossing Rates in Organic Emitters for Thermally Activated Delayed Fluorescence: Impact of the Nature of Singlet vs Triplet Excited States. J. Am. Chem. Soc. 2017, $139,4042-4051$.

(31) de Silva, P.; Kim, C. A.; Zhu, T.; Van Voorhis, T. Extracting Design Principles for Efficient Thermally Activated Delayed Fluorescence (TADF) from a Simple Four-State Model. Chem. Mater. 2019, 31, 6995-7006.

(32) Northey, T.; Stacey, J.; Penfold, T. J. The role of solid state solvation on the charge transfer state of a thermally activated delayed fluorescence emitter. J. Mater. Chem. C 2017, 5, 11001-11009.

(33) Evans, E. W.; Olivier, Y.; Puttisong, Y.; Myers, W. K.; Hele, T. J. H.; Menke, S. M.; Thomas, T. H.; Credgington, D.; Beljonne, D.; Friend, R. H. et al. Vibrationally Assisted Intersystem Crossing in Benchmark Thermally Activated Delayed Fluorescence Molecules. J. Phys. Chem. Lett. 2018, 9, 4053-4058, PMID: 29957961.

(34) Hu, T.; Han, G.; Tu, Z.; Duan, R.; Yi, Y. Origin of High Efficiencies for Thermally Activated Delayed Fluorescence Organic Light-Emitting Diodes: Atomistic Insight into Molecular Orientation and Torsional Disorder. J. Phys. Chem. C 2018, 122, 2719127197.

(35) Zhang, Q.; Li, B.; Huang, S.; Nomura, H.; Tanaka, H.; Adachi, C. Efficient blue organic light-emitting diodes employing thermally activated delayed fluorescence. Nat. Photonics 2014, 8, 326-332.

(36) Hilborn, R. C. Einstein coefficients, cross sections, $f$ values, dipole moments, and all that. Am. J. Phys. 1982, 50, 982-986.

(37) Marcus, R. A. On the Theory of Oxidation-Reduction Reactions Involving Electron Transfer. I. J. Chem. Phys. 1956, 24, 966-978. 
(38) Marcus, R. A. Chemical and Electrochemical Electron-Transfer Theory. Annu. Rev. Phys. Chem. 1964, 15, 155-196.

(39) Jortner, J. Temperature dependent activation energy for electron transfer between biological molecules. J. Chem. Phys. 1976, 64, 4860-4867.

(40) Sun, H.; Zhong, C.; Brdas, J.-L. Reliable Prediction with Tuned Range-Separated Functionals of the SingletTriplet Gap in Organic Emitters for Thermally Activated Delayed Fluorescence. J. Chem. Theory Comput. 2015, 11, 3851-3858, PMID: 26574466.

(41) Mewes, J.-M. Modeling TADF in organic emitters requires a careful consideration of the environment and going beyond the FranckCondon approximation. Phys. Chem. Chem. Phys. 2018, 20, 12454-12469.

(42) Saigo, M.; Miyata, K.; Tanaka, S.; Nakanotani, H.; Adachi, C.; Onda, K. Suppression of Structural Change upon S1T1 Conversion Assists the Thermally Activated Delayed Fluorescence Process in Carbazole-Benzonitrile Derivatives. J. Phys. Chem. Lett. 2019, $10,2475-2480$.

(43) Vosko, S. H.; Wilk, L.; Nusair, M. Accurate spin-dependent electron liquid correlation energies for local spin density calculations: a critical analysis. Can. J. Phys. 1980, 58, $1200-1211$.

(44) Becke, A. D. Density-functional exchange-energy approximation with correct asymptotic behavior. Phys. Rev. A 1988, 38, 3098-3100.

(45) Lee, C.; Yang, W.; Parr, R. G. Development of the Colle-Salvetti correlation-energy formula into a functional of the electron density. Phys. Rev. B 1988, 37, 785-789.

(46) Becke, A. D. Density-functional thermochemistry. III. The role of exact exchange. $J$. Chem. Phys. 1993, 98, 5648-5652. 
(47) Stephens, P. J.; Devlin, F. J.; Chabalowski, C. F.; Frisch, M. J. Ab Initio Calculation of Vibrational Absorption and Circular Dichroism Spectra Using Density Functional Force Fields. J. Phys. Chem. 1994, 98, 11623-11627.

(48) Kim, K.; Jordan, K. D. Comparison of Density Functional and MP2 Calculations on the Water Monomer and Dimer. J. Phys. Chem. 1994, 98, 10089-10094.

(49) Hariharan, P. C.; Pople, J. A. The influence of polarization functions on molecular orbital hydrogenation energies. Theor. Chim. Acta 1973, 28, 213-222.

(50) Krishnan, R.; Binkley, J. S.; Seeger, R.; Pople, J. A. Self-consistent molecular orbital methods. XX. A basis set for correlated wave functions. J. Chem. Phys. 1980, 72, $650-654$.

(51) Francl, M. M.; Pietro, W. J.; Hehre, W. J.; Binkley, J. S.; Gordon, M. S.; DeFrees, D. J.; Pople, J. A. Self-consistent molecular orbital methods. XXIII. A polarization-type basis set for second-row elements. J. Chem. Phys. 1982, 77, 3654-3665.

(52) Clark, T.; Chandrasekhar, J.; Spitznagel, G. W.; Schleyer, P. V. R. Efficient diffuse function-augmented basis sets for anion calculations. III. The $3-21+\mathrm{G}$ basis set for first-row elements, Li-F. J. Comput. Chem. 1983, 4, 294-301.

(53) Shao, Y.; Gan, Z.; Epifanovsky, E.; Gilbert, A. T. B.; Wormit, M.; Kussmann, J.; Lange, A. W.; Behn, A.; Deng, J.; Feng, X. et al. Advances in molecular quantum chemistry contained in the Q-Chem 4 program package. Mol. Phys. 2015, 113, 184215.

(54) Dreuw, A.; Head-Gordon, M. Failure of Time-Dependent Density Functional Theory for Long-Range Charge-Transfer Excited States: The Zincbacteriochlorin-Bacteriochlorin and Bacteriochlorophyll-Spheroidene Complexes. J. Am. Chem. Soc. 2004, 126, 40074016. 
(55) Dreuw, A.; Head-Gordon, M. Single-Reference ab Initio Methods for the Calculation of Excited States of Large Molecules. Chem. Rev. 2005, 105, 4009-4037.

(56) Perdew, J. P.; Burke, K.; Ernzerhof, M. Generalized Gradient Approximation Made Simple. Phys. Rev. Lett. 1996, 77, 3865-3868.

(57) Perdew, J. P.; Ernzerhof, M.; Burke, K. Rationale for mixing exact exchange with density functional approximations. J. Chem. Phys. 1996, 105, 9982-9985.

(58) Adamo, C.; Barone, V. Toward reliable density functional methods without adjustable parameters: The PBE0 model. J. Chem. Phys. 1999, 110, 6158-6170.

(59) Zhao, Y.; Truhlar, D. G. The M06 suite of density functionals for main group thermochemistry, thermochemical kinetics, noncovalent interactions, excited states, and transition elements: two new functionals and systematic testing of four M06-class functionals and 12 other functionals. Theor. Chem. Acc. 2008, 120, 215-241.

(60) Rohrdanz, M. A.; Herbert, J. M. Simultaneous benchmarking of ground- and excitedstate properties with long-range-corrected density functional theory. J. Chem. Phys. 2008, 129, 034107.

(61) Henderson, T. M.; Janesko, B. G.; Scuseria, G. E. Generalized gradient approximation model exchange holes for range-separated hybrids. J. Chem. Phys. 2008, 128, 194105.

(62) Salzner, U.; Baer, R. Koopmans springs to life. J. Chem. Phys 2009, 131, 231101.

(63) Cancs, E.; Mennucci, B.; Tomasi, J. A new integral equation formalism for the polarizable continuum model: Theoretical background and applications to isotropic and anisotropic dielectrics. J. Chem. Phys. 1997, 107, 3032-3041.

(64) Chipman, D. M. Reaction field treatment of charge penetration. J. Chem. Phys. 2000, 112, 5558-5565. 
(65) Cancs, E.; Mennucci, B. Comment on Reaction field treatment of charge penetration [J. Chem. Phys. 112, 5558 (2000)]. J. Chem. Phys. 2001, 114, 4744-4745.

(66) Mewes, J.-M.; You, Z.-Q.; Wormit, M.; Kriesche, T.; Herbert, J. M.; Dreuw, A. Experimental Benchmark Data and Systematic Evaluation of Two a Posteriori, PolarizableContinuum Corrections for Vertical Excitation Energies in Solution. The Journal of Physical Chemistry A 2015, 119, 5446-5464, PMID: 25629414.

(67) Marchal, J.; Lapp, C. Dielectric properties of polymethyl methacrylate in dilute solution previous results. J. Polym. Sci. 1958, 27, 571-573.

(68) Hanwell, M. D.; Curtis, D. E.; Lonie, D. C.; Vandermeersch, T.; Zurek, E.; Hutchison, G. R. Avogadro: an advanced semantic chemical editor, visualization, and analysis platform. J. Cheminformatics 2012, 4, 17.

(69) Martin, R. L. Natural transition orbitals. J. Chem. Phys. 2003, 118, 4775-4777.

(70) Bode, B. M.; Gordon, M. S. MacMolPlt: a graphical user interface for GAMESS. J. Mol. Graph. Model. 1998, 16, 133-8.

(71) Spendley, W.; Hext, G. R.; Himsworth, F. R. Sequential Application of Simplex Designs in Optimisation and Evolutionary Operation. Technometrics 1962, 4, 441-461.

(72) Nelder, J. A.; Mead, R. A Simplex Method for Function Minimization. Comput. J. 1965, 7, 308-313.

(73) Lee, S. Y.; Yasuda, T.; Yang, Y. S.; Zhang, Q.; Adachi, C. Luminous Butterflies: Efficient Exciton Harvesting by Benzophenone Derivatives for Full-Color Delayed Fluorescence OLEDs. Angew. Chem. Int. Ed. 2014, 53, 6402-6406.

(74) Komatsu, R.; Sasabe, H.; Seino, Y.; Nakao, K.; Kido, J. Light-blue thermally activated delayed fluorescent emitters realizing a high external quantum efficiency of $25 \%$ and unprecedented low drive voltages in OLEDs. J. Mater. Chem. C 2016, 4, 2274-2278. 
(75) Huang, W.; Einzinger, M.; Zhu, T.; Chae, H. S.; Jeon, S.; Ihn, S.-G.; Sim, M.; Kim, S.; Su, M.; Teverovskiy, G. et al. Molecular Design of Deep Blue Thermally Activated Delayed Fluorescence Materials Employing a Homoconjugative Triptycene Scaffold and Dihedral Angle Tuning. Chem. Mater. 2018, 30, 1462-1466.

(76) Huang, W.; Einzinger, M.; Maurano, A.; Zhu, T.; Tiepelt, J.; Yu, C.; Chae, H. S.; Van Voorhis, T.; Baldo, M. A.; Buchwald, S. L. Large Increase in External Quantum Efficiency by Dihedral Angle Tuning in a Sky-Blue Thermally Activated Delayed Fluorescence Emitter. Adv. Opt. Mater. 2019, 7, 1900476.

(77) Zhan, L.; Xiang, Y.; Chen, Z.; Wu, K.; Gong, S.; Xie, G.; Yang, C. Fine-tuning the photophysical properties of thermally activated delayed fluorescent emitters using torsion angles: high performance sky-blue OLEDs. J. Mater. Chem. C 2019, 7, 13953-13959.

(78) Ohlendorf, G.; Mahler, C. W.; Jester, S.-S.; Schnakenburg, G.; Grimme, S.; Hger, S. Highly Strained Phenylene Bicyclophanes. Angew. Chem. Int. Edit. 2013, 52, 1208612090.

(79) Liu, Y.-Y.; Lin, J.-Y.; Bo, Y.-F.; Xie, L.-H.; Yi, M.-D.; Zhang, X.-W.; Zhang, H.-M.; Loh, T.-P.; Huang, W. Synthesis and Crystal Structure of Highly Strained [4]Cyclofluorene: Green-Emitting Fluorophore. Org. Lett. 2016, 18, 172-175, PMID: 26695881.

(80) Penfold, T. J. On Predicting the Excited-State Properties of Thermally Activated Delayed Fluorescence Emitters. J. Phys. Chem. C 2015, 119, 13535-13544. 


\section{TOC Graphic}

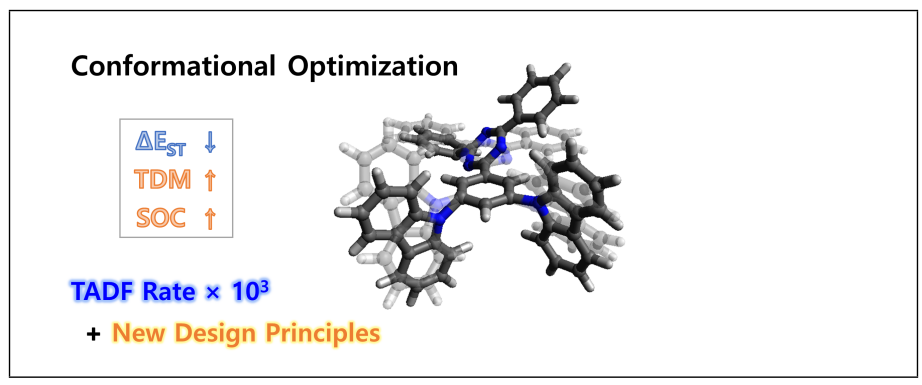




\title{
Supporting Information:
}

\section{Maximizing TADF via Conformational \\ Optimization}

\author{
Changhae Andrew Kim and Troy Van Voorhis* \\ Department of Chemistry, Massachusetts Institute of Technology, Cambridge, \\ Massachusetts 02139, United States \\ E-mail: tvan@mit.edu
}

\section{List of Figures}

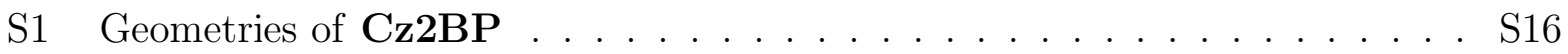

S2 Geometries of Ac-MPM . . . . . . . . . . . . . . S17

S3 Geometries of SpiroAC-TRZ . . . . . . . . . . . . . . . . S19

S4 NTOs of DCzTrz . . . . . . . . . . . . . . . . . . . . . . S S21

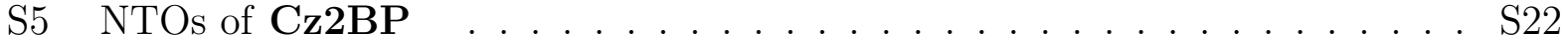

S6 NTOs of Ac-MPM . . . . . . . . . . . . . . . . . . . . . S S22

S7 NTOs of SpiroAC-TRZ . . . . . . . . . . . . . . . . . . . . S23

\section{List of Tables}

S1 Energetics of the model systems at the maximum TADF geometries . . . . S S

S2 Energetics of the model systems recomputed using PBE0 . . . . . . . . . S11 
S3 Energetics of the model systems recomputed using M06-2X . . . . . . . S S12

S4 Energetics of the model systems recomputed using LRC- $\omega^{*} \mathrm{PBE} \ldots$. . . . . S13

S5 Energetics of the model systems recomputed using M06-2X/IEF-PCM . . . S14

S6 Energetics of the model systems recomputed using LRC- $\omega^{*}$ PBE/IEF-PCM . S15

S7 Z-matrix of DCzTrz at the energy minimum $(\Lambda=\infty) \ldots \ldots \ldots$ S24

S8 Z-matrix of DCzTrz at the TADF maximum with energy penalty $(\Lambda=$

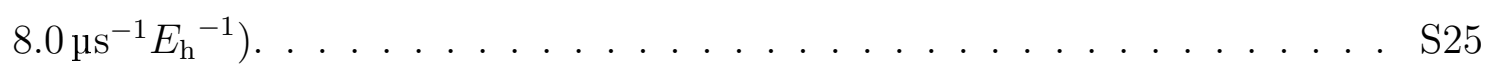

S9 Z-matrix of DCzTrz at the TADF maximum with energy penalty $(\Lambda=$

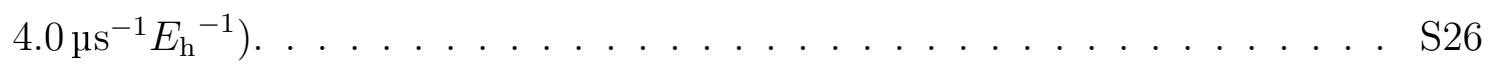

S10 Z-matrix of DCzTrz at the TADF maximum with energy penalty $(\Lambda=$

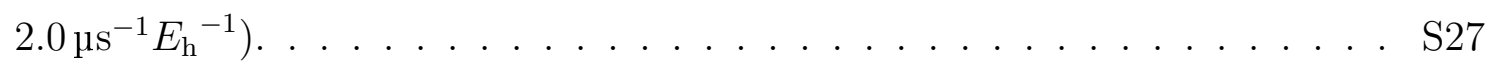

S11 Z-matrix of DCzTrz at the TADF maximum with energy penalty $(\Lambda=$

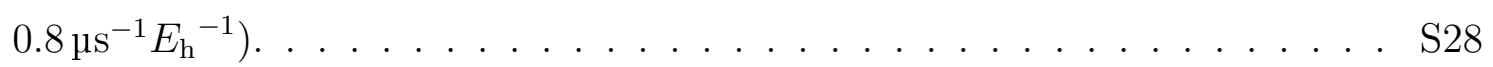

S12 Z-matrix of DCzTrz at the TADF maximum with no energy penalty $(\Lambda=0)$. S29

S13 Z-matrix of DCzTrz at the TADF maximum $(\Lambda=0)$ after the relaxation of the hydrogen atoms and the aromatic ring systems. . . . . . . . . S30

S14 Z-matrix of Cz2BP at the energy minimum $(\Lambda=\infty) \ldots \ldots \ldots$ S31

S15 Z-matrix of Cz2BP at the TADF maximum with energy penalty $(\Lambda=$

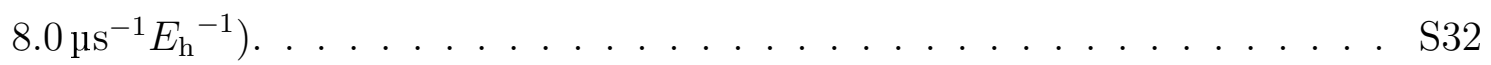

S16 Z-matrix of Cz2BP at the TADF maximum with energy penalty $(\Lambda=$ $\left.4.0 \mathrm{ps}^{-1} E_{\mathrm{h}}^{-1}\right) \ldots \ldots \ldots \ldots \ldots \ldots \ldots \ldots \ldots \ldots \ldots \ldots \ldots \ldots$

S17 Z-matrix of Cz2BP at the TADF maximum with no energy penalty $(\Lambda=0)$. S34

S18 Z-matrix of Cz2BP at the TADF maximum $(\Lambda=0)$ after the relaxation of the hydrogen atoms and the aromatic ring systems. . . . . . . . . . . S35

S19 Z-matrix of Ac-MPM at the energy minimum $(\Lambda=\infty) \ldots \ldots$. . . . S36

S20 Z-matrix of Ac-MPM at the TADF maximum with energy penalty $(\Lambda=$ $\left.8.0 \mathrm{ps}^{-1} E_{\mathrm{h}}{ }^{-1}\right) \ldots \ldots \ldots \ldots \ldots \ldots \ldots \ldots \ldots \ldots \ldots \ldots \ldots \ldots \ldots$ 
S21 Z-matrix of Ac-MPM at the TADF maximum with energy penalty $(\Lambda=$ $\left.4.0 \mathrm{ps}^{-1} E_{\mathrm{h}}{ }^{-1}\right) \ldots \ldots \ldots \ldots \ldots \ldots \ldots \ldots \ldots$

S22 Z-matrix of Ac-MPM at the TADF maximum with no energy penalty $(\Lambda=0)$.S39

S23 Z-matrix of Ac-MPM at the TADF maximum $(\Lambda=0)$ after the relaxation of the hydrogen atoms and the aromatic ring systems. . . . . . . . . . . S40

S24 Z-matrix of SpiroAC-TRZ at the energy minimum $(\Lambda=\infty) \ldots . .$. S41

S25 Z-matrix of SpiroAC-TRZ at the TADF maximum with energy penalty

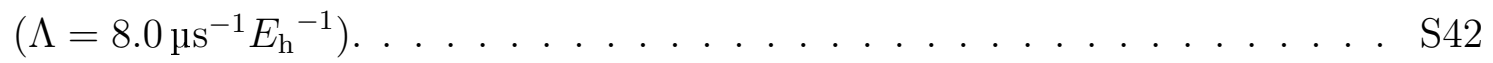

S26 Z-matrix of SpiroAC-TRZ at the TADF maximum with energy penalty $\left(\Lambda=4.0 \mathrm{us}^{-1} E_{\mathrm{h}}^{-1}\right) \ldots \ldots \ldots \ldots \ldots \ldots \ldots \ldots$

S27 Z-matrix of SpiroAC-TRZ at the TADF maximum with no energy penalty $(\Lambda=0) \ldots \ldots \ldots \ldots \ldots \ldots \ldots \ldots \ldots$

S28 Z-matrix of SpiroAC-TRZ at the TADF maximum $(\Lambda=0)$ after the relaxation of the hydrogen atoms and the aromatic ring systems. . . . . . . . . S45 


\section{A Variation of the Nelder-Mead Simplex Method}

We want to maximize the TADF rate of a molecule in a subspace of the Z-matrix coordinates. Let $\mathbf{x}_{0}$ be the vector of the $n$ coordinates at the initial geometry.

1. Initialization Prepare $n+1$ geometries $\mathbf{x}_{0}, \mathbf{x}_{1}, \ldots, \mathbf{x}_{n}$, where $\mathbf{x}_{i>0}$ are generated by displacing the $i$-th coordinate of $\mathbf{x}_{0}$. We displaced the bond lengths by $0.05 \AA$ and the bond and the dihedral angles by $5.0^{\circ}$. The geometries correspond to the vertices of a $n$-simplex. Calculate the electronic structure at each geometry, and evaluate the TADF rate.

2. Termination Compute the center of the simplex $\mathbf{x}_{c}$. If every coordinate at every vertex is within some tolerance, then the maximization has converged. We required the bond lengths to be within $0.005 \AA$ and the bond and dihetral angles to be within $0.5^{\circ}$ of the corresponding value at the center.

3. Reflection, Expansion, and Contraction Sort the vertices in the order of descending TADF rate. Let $\mathbf{x}_{n}$ denote the worst vertex. Compute the center of the facet $\mathbf{x}_{o}$ consisting of the first $n$ vertices. Sample candidate vertices on the line $\mathbf{x}_{0}+\alpha\left(\mathbf{x}_{n}-\mathbf{x}_{o}\right)$. We chose four points: $\alpha=-0.5,+0.5,+1.0,+2.0$. Calculate the electronic structure at each geometry, and evaluate the TADF rate.

4. Shrink Sort the candidate vertices in the order of descending TADF rate. Let $\mathbf{x}_{n}^{\prime}$ denote the best candidate vertex. If $\mathbf{x}_{n}^{\prime}$ has a higher TADF rate than $\mathbf{x}_{n-1}$, then replace $\mathbf{x}_{n}$ with $\mathbf{x}_{n}^{\prime}$ and return to step 2. Otherwise, replace $\mathbf{x}_{i>0}$ with $0.5 \cdot\left(\mathbf{x}_{i>0}+\mathbf{x}_{0}\right)$, calculate the electronic structure at each geometry, evaluate the TADF rate, and return to step 2.

A notable feature of the above algorithm is that the function evaluations corresponding to Reflection, Expansion, and Contraction are performed in parallel, whereas they are performed in series in the original algorithm of Nelder and Mead. ${ }^{1}$ The parallel algorithm does not make the most efficient use of the CPU time, since the serial algorithm would use heuristics to avoid function evaluations at one or more of the candidate vertices. However, 
we found that knowing the TADF rate of every candidate vertex can help convergence as the differences in the TADF rates approach the uncertainty of the electronic structure method. Moreover, even though the CPU time might increase, the wall time can be reduced by assigning each electronic structure calculation to a separate set of CPU cores. Depending on the number of molecules to optimize and the real world time constraints, either the serial or parallel algorithm might be more useful than the other. 


\section{Rate of Reverse Intersystem Crossing}

The rate of reverse intersystem crossing (RISC) was estimated using Fermi's golden rule

$$
k_{\mathrm{RISC}}^{\mathrm{T}_{n} \rightarrow \mathrm{S}_{1}}=\frac{2 \pi}{\hbar}\left|\left\langle\mathrm{S}_{1}\left|\hat{H}_{\mathrm{SO}}\right| \mathrm{T}_{n}\right\rangle\right|^{2} \rho_{\mathrm{FC}}^{\mathrm{T}_{n} \rightarrow \mathrm{S}_{1}}
$$

where $\rho_{\mathrm{FC}}^{\mathrm{T}_{n} \rightarrow \mathrm{S}_{1}}$ is the Franck-Condon weighted density of states (FCWD). One of the simplest ways to estimate the FCWD is the Marcus theory ${ }^{2,3}$

$$
\rho_{\mathrm{FC}}^{\mathrm{T}_{n} \rightarrow \mathrm{S}_{1}}=\frac{1}{\sqrt{4 \pi \lambda_{M} k_{B} T}} \exp \left[-\frac{\left(\Delta E_{\mathrm{S}_{1}-\mathrm{T}_{n}}+\lambda_{M}\right)^{2}}{4 \lambda_{M} k_{B} T}\right]
$$

where $\lambda_{M}$ is the Marcus reorganization energy. Since conformational optimization considers a single conformer of a definite geometry, it is incompatible with geometric relaxation. Hence, we just used a typical value of the reorganization energy $\lambda_{M}=0.2 \mathrm{eV} .{ }^{4-6}$ We expected that the simplified approach would give accuracies to the correct order of magnitude, which would suffice to assess the extent to which a finite RISC rate slows down TADF.

Since Eq. (2) of the main text represents the limit of fast RISC, we consider the opposite limit of slow RISC, i.e. $k_{\mathrm{RISC}} \ll k_{\mathrm{F}}+k_{\mathrm{ISC}}$. Assuming that internal conversion (IC) is fast enough that only the $\mathrm{S}_{1}, \mathrm{~T}_{1}$, and $\mathrm{T}_{2}$ states remain populated after a transient, the kinetic equation of the $\mathrm{S}_{1}$ state becomes

$$
\frac{d\left[\mathrm{~S}_{1}\right]}{d t}=-k_{\mathrm{F}}\left[\mathrm{S}_{1}\right]-k_{\mathrm{ISC}}^{\mathrm{S}_{1} \rightarrow \mathrm{T}_{1}}\left[\mathrm{~S}_{1}\right]+k_{\mathrm{RISC}}^{\mathrm{T}_{1} \rightarrow \mathrm{S}_{1}}\left[\mathrm{~T}_{1}\right]-k_{\mathrm{ISC}}^{\mathrm{S}_{1} \rightarrow \mathrm{T}_{2}}\left[\mathrm{~S}_{1}\right]+k_{\mathrm{RISC}}^{\mathrm{T}_{2} \rightarrow \mathrm{S}_{1}}\left[\mathrm{~T}_{2}\right]
$$

Furthermore, assume that IC maintains a quasi-equilibrium between the triplet states with the equilibrium cosntant

$$
K_{12}=\exp \left(\frac{\Delta E_{\mathrm{T}_{1}-\mathrm{T}_{2}}}{k_{B} T}\right)
$$


Then, the equation simplifies to

$$
\frac{d\left[\mathrm{~S}_{1}\right]}{d t}=-k_{\mathrm{F}}\left[\mathrm{S}_{1}\right]-k_{\mathrm{ISC}}\left[\mathrm{S}_{1}\right]+k_{\mathrm{RISC}}\left[\mathrm{T}_{1}\right]
$$

where $k_{\mathrm{ISC}}=k_{\mathrm{ISC}}^{\mathrm{S}_{1} \rightarrow \mathrm{T}_{1}}+k_{\mathrm{ISC}}^{\mathrm{S}_{1} \rightarrow \mathrm{T}_{2}}$ and $k_{\mathrm{RISC}}=k_{\mathrm{RISC}}^{\mathrm{T}_{1} \rightarrow \mathrm{S}_{1}}+k_{\mathrm{RISC}}^{\mathrm{T}_{2} \rightarrow \mathrm{S}_{1}} K_{21}$. Using the steady state approximation, we extract the quasi-steady state population of the $\mathrm{S}_{1}$ state

$$
\frac{\left[\mathrm{S}_{1}\right]}{\left[\mathrm{T}_{1}\right]}=\frac{k_{\mathrm{RISC}}}{k_{\mathrm{F}}+k_{\mathrm{ISC}}}
$$

Returning to the definition of the TADF rate, we rearrange the expression

$$
k_{\mathrm{TADF}} \equiv \frac{k_{\mathrm{F}}\left[\mathrm{S}_{1}\right]}{\left[\mathrm{S}_{1}\right]+\left[\mathrm{T}_{1}\right]+\left[\mathrm{T}_{2}\right]}=\left[\frac{1}{k_{\mathrm{F}}}+\frac{1+K_{12}}{k_{\mathrm{F}}} \cdot \frac{\left[\mathrm{T}_{1}\right]}{\left[\mathrm{S}_{1}\right]}\right]^{-1}
$$

Substituting Eq. (S6) and rearranging to gives

$$
k_{\mathrm{TADF}}=\left[\frac{1}{k_{\mathrm{F}}}+\frac{1+K_{12}}{k_{\mathrm{F}}} \cdot \frac{k_{\mathrm{ISC}}}{k_{\mathrm{RISC}}}+\frac{1+K_{12}}{k_{\mathrm{RISC}}}\right]^{-1}
$$

Detailed balance among the $\mathrm{S}_{1}, \mathrm{~T}_{1}$, and $\mathrm{T}_{2}$ states implies that $k_{\mathrm{ISC}} / k_{\mathrm{RISC}}=K_{1}$. The TADF rate becomes

$$
k_{\mathrm{TADF}}=\left[\frac{1+K_{1}+K_{2}}{k_{\mathrm{F}}}+\frac{1+K_{12}}{k_{\mathrm{RISC}}^{\mathrm{T}_{1} \rightarrow \mathrm{S}_{1}}+k_{\mathrm{RISC}}^{\mathrm{T}_{2} \rightarrow \mathrm{S}_{1}} K_{12}}\right]^{-1}
$$

where we have also used $K_{12}=K_{2} / K_{1}$ and $k_{\mathrm{RISC}}=k_{\mathrm{RISC}}^{\mathrm{T}_{1} \rightarrow \mathrm{S}_{1}}+k_{\mathrm{RISC}}^{\mathrm{T}_{2} \rightarrow \mathrm{S}_{1}} K_{21}$. Interestingly, Eq. (2) of the main text appears in an intact form inside Eq. (S9), and the RISC correction is additive to the reciprocal of the idealized TADF rate. For the assumption of fast RISC to be valid, the RISC rate only needs to be fast compared to the idealized TADF rate, which is much easier than fast compared to the fluorescence rate.

Table S1 summarizes the energetics of the model systems at the maximum TADF geometries with various energy penalties. The electronic structures were calculated using the B3LYP exchange-correlation functional ${ }^{7-12}$ and the $6-31+\mathrm{G}^{*}$ basis set, ${ }^{13-16}$ and the spin- 


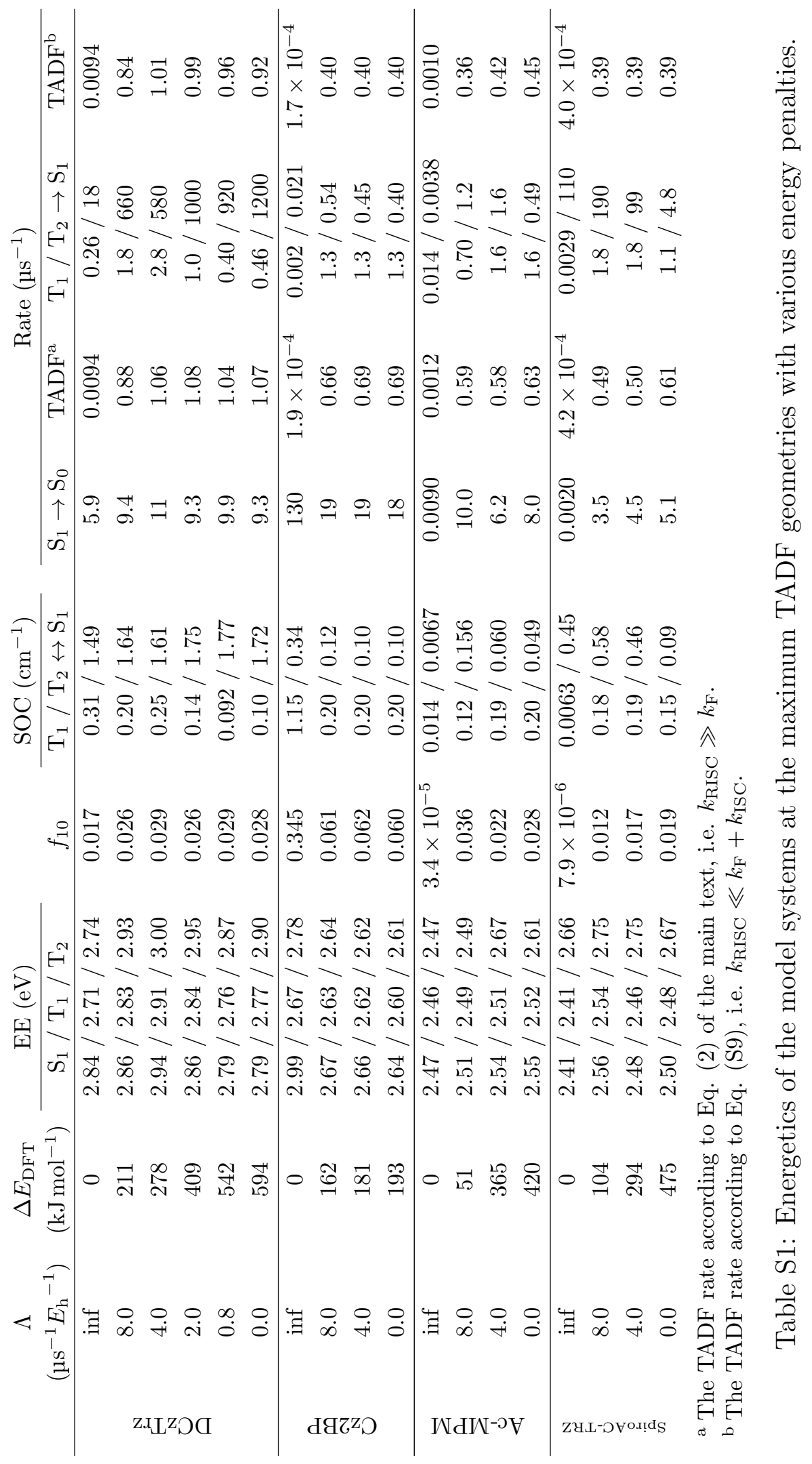


orbit coupling (SOC) matrix elements were computed using the one-electron Breit-Pauli Hamiltonian as implemented in the Q-Chem 5.1 software package. ${ }^{17}$ Among the optimized geometries, the RISC correction entails upto $42 \%$ decrease in the TADF rate. Although $42 \%$ might seem like a large change, the RISC correction is insignificant compared to the orders-of-magnitude enhancement in the TADF rate between the energy minimum and the TADF maximum. Besides, the RISC correction is less than $5 \%$ in most cases, and one of the DCzTrz geometries continue to exhibit a TADF rate in the submicrosecond timescales. The RISC correction might be important in a quantitative prediction of the TADF rate, but it does not alter the qualitative outcomes of conformational optimization.

It is remarkable that the RISC rate increases along with the idealized TADF rate, even though the TADF rate maximization did not consider the RISC rate in an explicit manner. The simultaneous enhancement of the RISC rate makes sense, given the parallel between the idealized TADF rate and the RISC rate: a high RISC rate requires a small singlet-triplet (ST) gap and a large SOC matrix element, which are expected to be contraindicated functions of the HOMO-LUMO overlap. Changes to the HOMO and the LUMO that increase the transition dipole moment (TDM) are likely to also increase the SOC matrix element, though the increases need not be proportional. Indeed, the TDMs of Ac-MPM and SpiroACTRZ increase by factors of 1000 and 2000, whereas the SOC matrix elements increase by factors of 15 and 25, respectively. On the other extreme, the RISC step appears even less likely to be the bottleneck in the TADF process as the ST gap becomes smaller. As shown by the minimum energy conformations of Ac-MPM and SpiroAC-TRZ, the $\mathrm{T}_{1} \rightarrow \mathrm{S}_{1}$ RISC rate alone is enough to overtake the prompt fluorescence rate in the regime of vanishing ST gap.

Finally, as Brédas et al. have pointed out ${ }^{6}$ the $\mathrm{T}_{2}$ state appears to make an important contribution to the total RISC rate in some systems. In the cases of DCzTrz and SpiroACTRZ, the $T_{2} \rightarrow S_{1}$ RISC rates are orders of magnitude greater than the respective $T_{1} \rightarrow S_{1}$ RISC rates. Even if the $T_{2}$ state is higher in energy than the $T_{1}$ and the $S_{1}$ states, the $T_{2}$ 
contributions to the total RISC rate can be non-negligible.

Tables S2-S4 summarize the energetics of the model systems, recomputed using PBE0 ${ }^{18-20}$ M06-2X, ${ }^{21}$ and LRC- $\omega^{*}$ PBE. ${ }^{22-24}$ Tables S5 and S6 are the outcomes of M06-2X and LRC$\omega^{*} \mathrm{PBE}$ with integral equation formalism polarizable continuum model (IEF-PCM) ${ }^{25-27}$ and first order, perturbative state-specific (ptSS) ${ }^{28}$ treatment of the excited states. We used the dielectric constant and the optical dielectric constant of toluene at $25^{\circ} \mathrm{C}: \varepsilon=2.379$ and $\varepsilon_{\infty}=2.232 .{ }^{29}$ Remember that we have not reoptimized the geometries and just recalculated the electronic structures.

It is remarkable that the various functionals exhibit such similar behaviors. For maximum TADF geometries with little or no energy penalties, the functionals predict that the TADF rate would be similar to or even worse than the the minimum energy geometry. For maximum TADF geometries with sufficient energy penalties $\left(\Lambda \geq 4.0 \mathrm{\mu s}^{-1} E_{\mathrm{h}}{ }^{-1}\right)$, the functionals agree with B3LYP that the TADF rate could be orders of magnitude greater than the minimum energy geometry. It would seem that the TADF rate maximization tended to exploit some quirk of the B3LYP functional, but the imposition of energy penalties mitigated the issue.

It is also remarkable that different molecules exhibit a simultaneous improvement of the ST gap and TDM in different functionals. The simulataneous improvement is not observed in M06-2X and LRC- $\omega^{*} \mathrm{PBE}$ without solvent models, but in M06-2X/IEF-PCM and LRC$\omega^{*} \mathrm{PBE} / \mathrm{IEF}-\mathrm{PCM}$ with a toluene-like environment. In the second case, the maximized TADF rates of Ac-MPM and SpiroAC-TRZ are quite impressive, as they are well within the submicrosecond timescales.

Finally, the various functional predict the RISC correction to be even less important than B3LYP did. With a few excpetions, the RISC corrections are less than $10 \%$ of the TADF rates. The finite rate of RISC does not seem to overturn the qualitative trends of conformational optimization. 


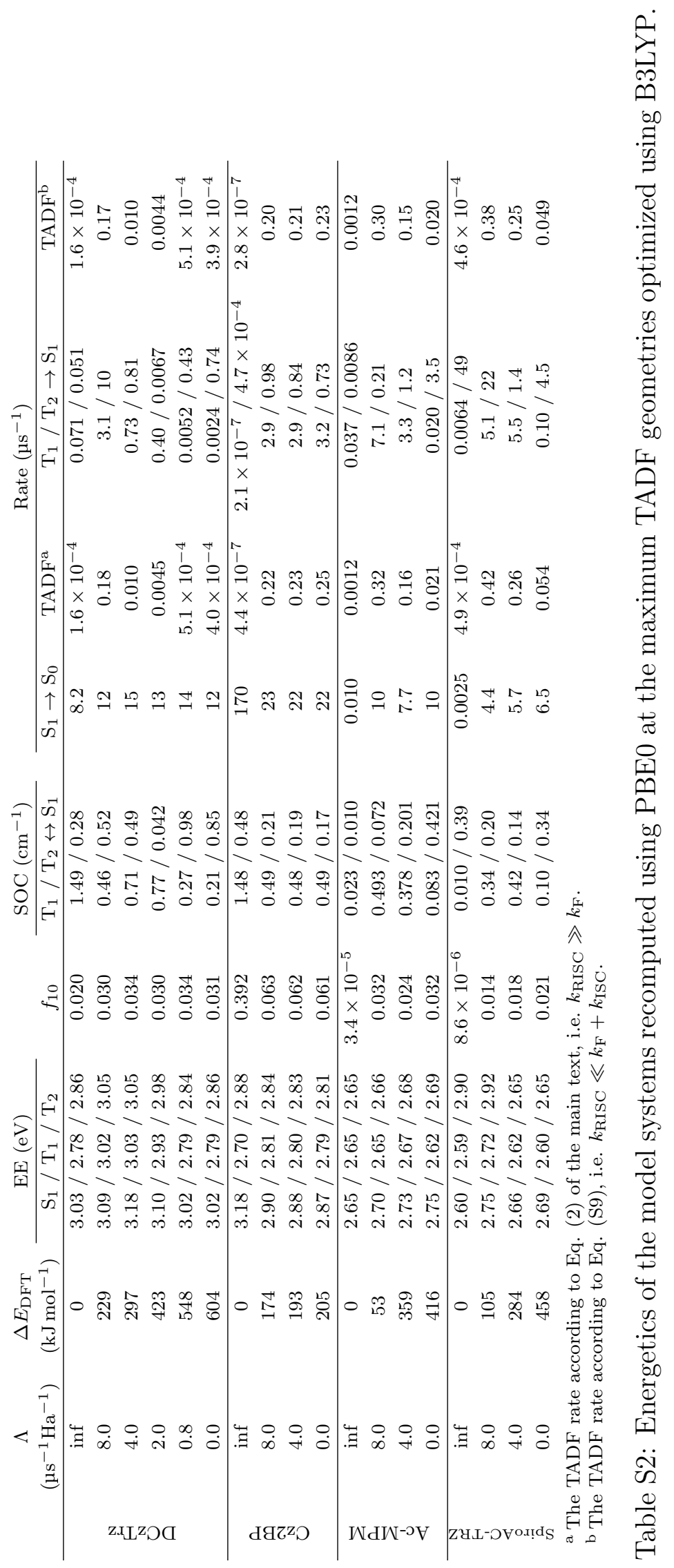




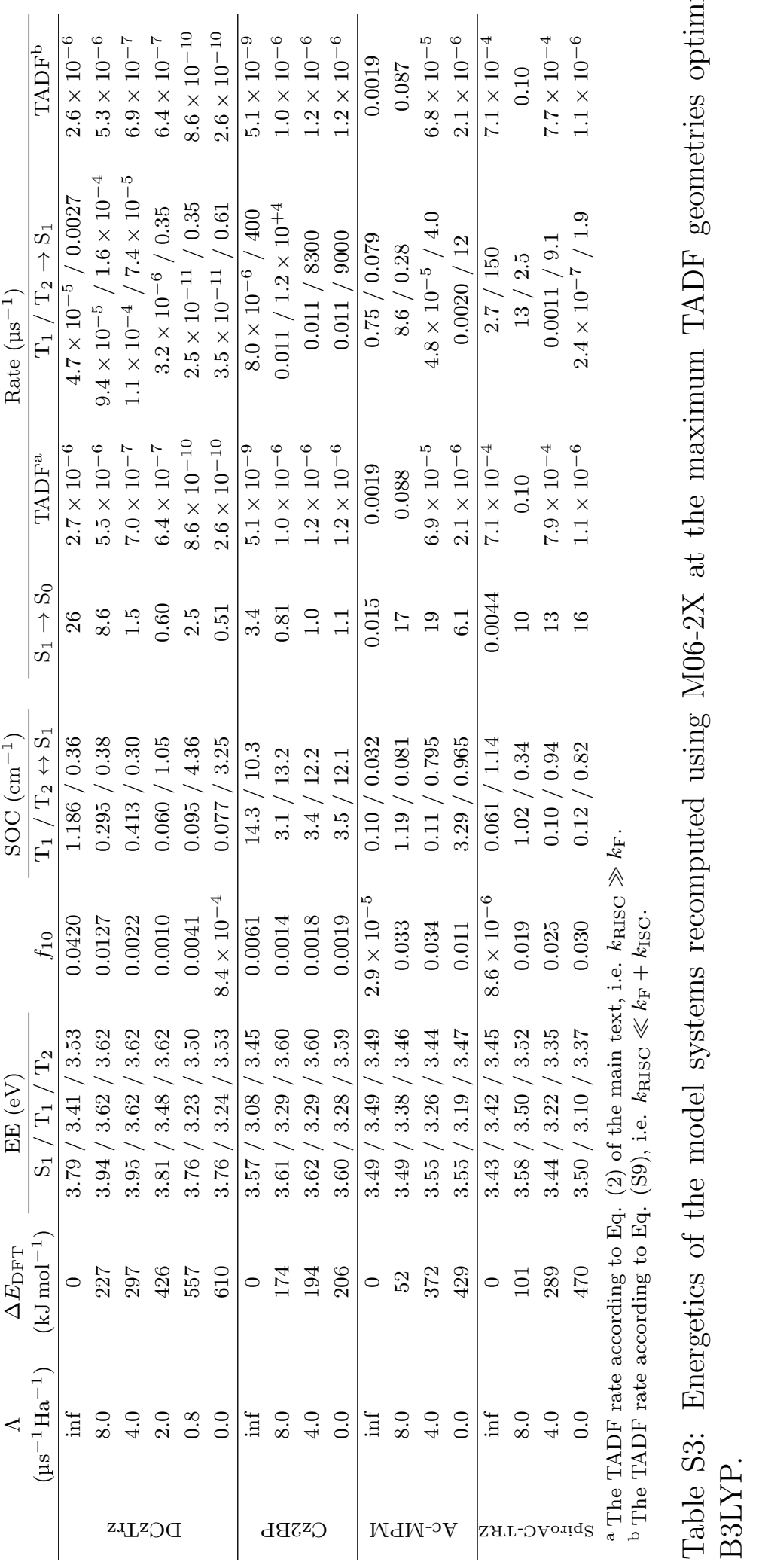




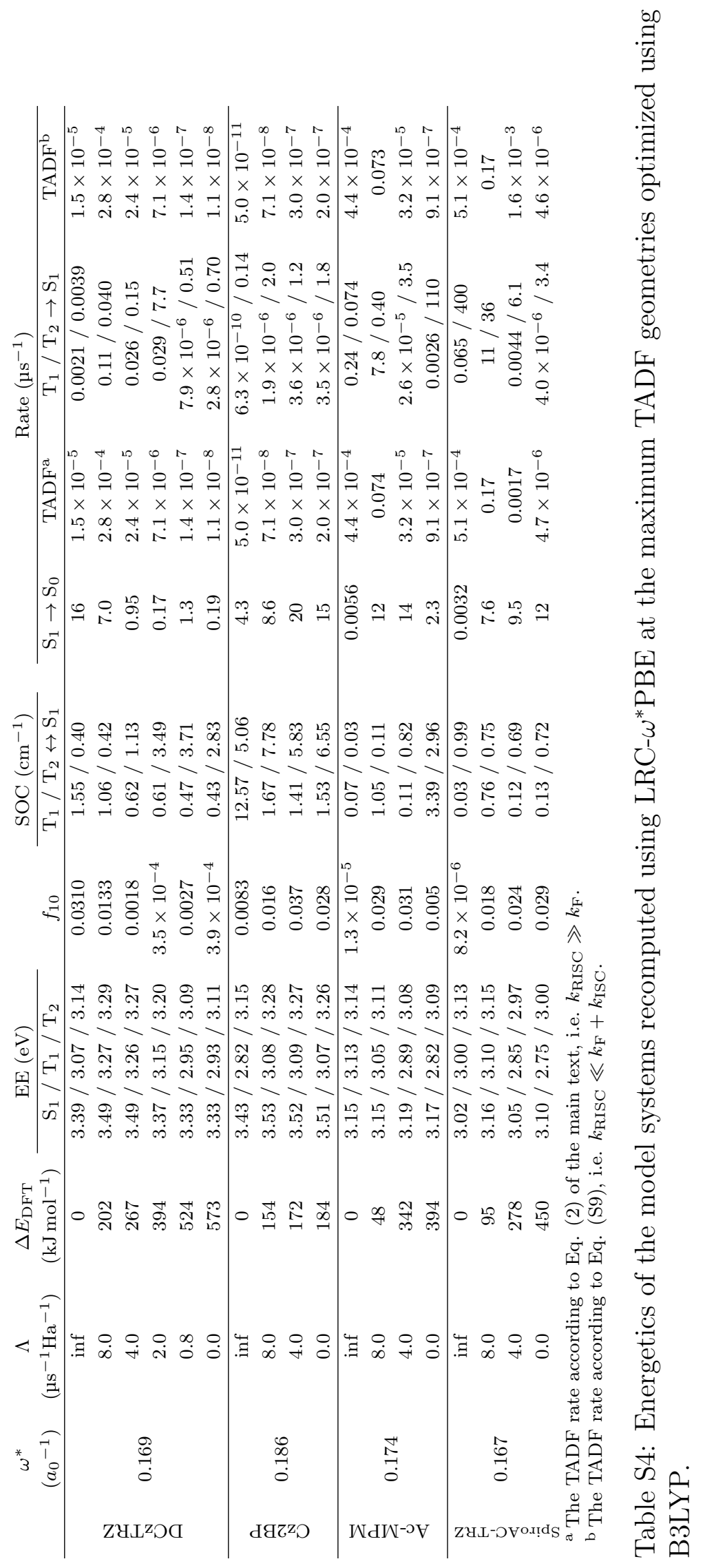




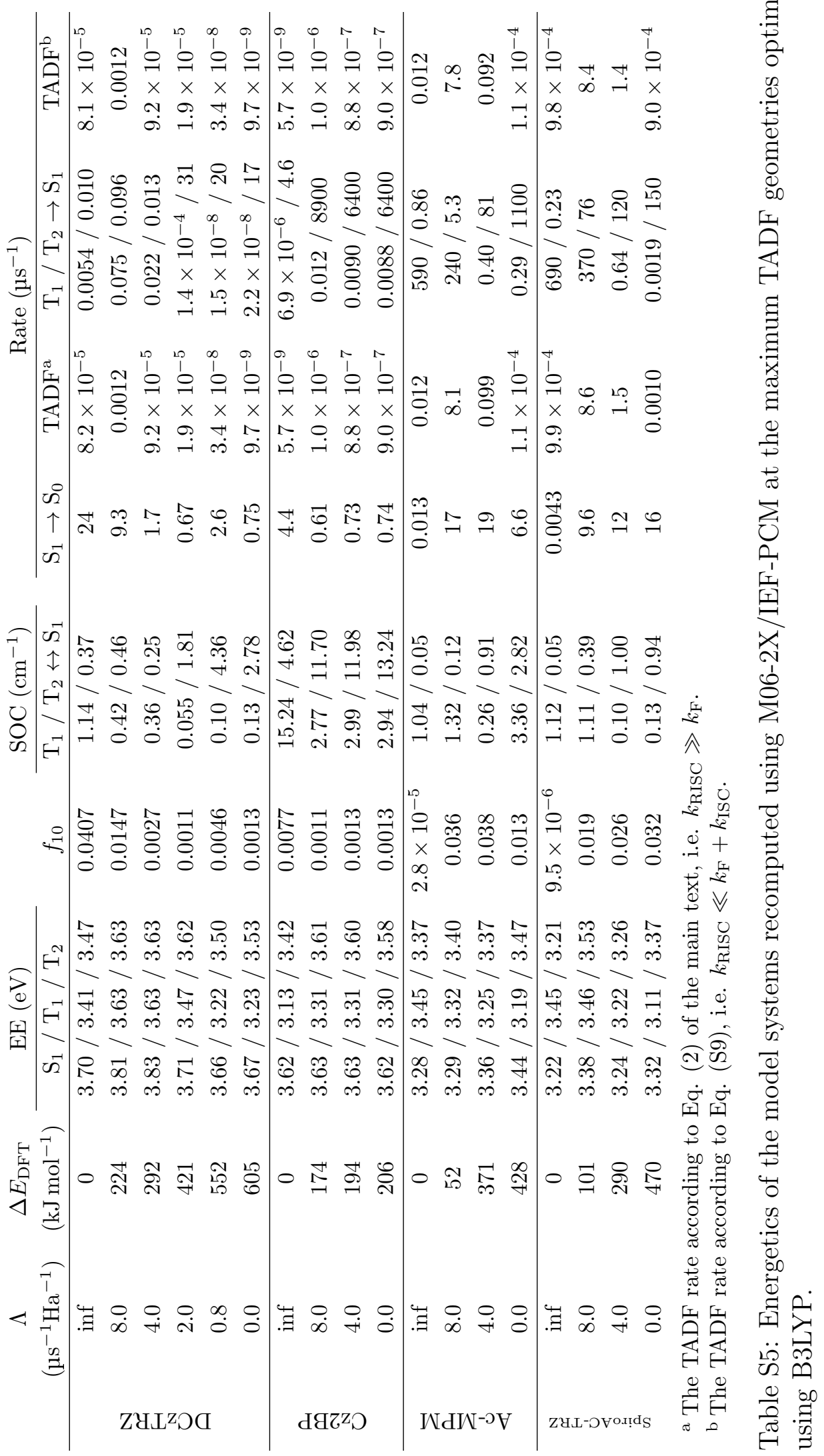




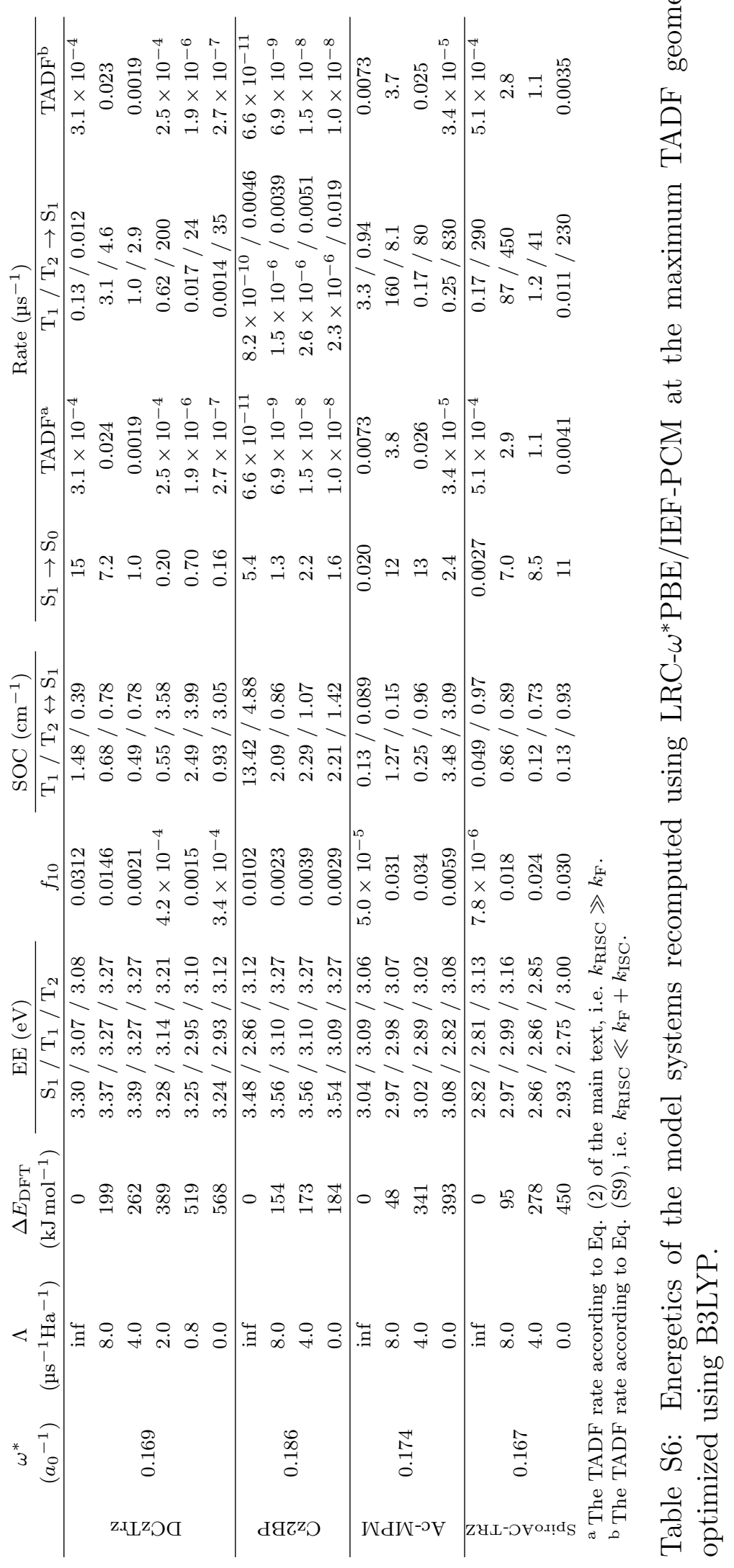




\section{Geometries of Cz2BP, Ac-MPM, and SpiroAC-TRZ}

The 3D images of the geometries were generated in Avogadro 1.2.0. ${ }^{30}$

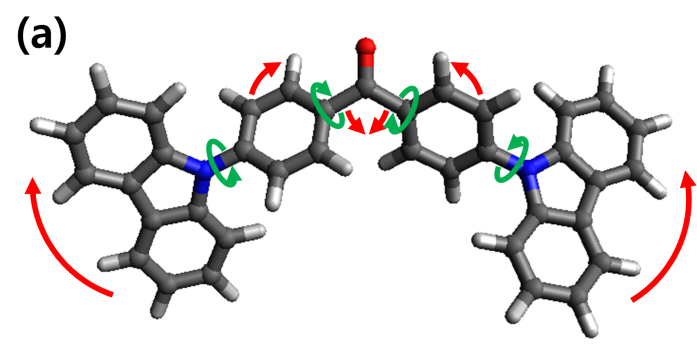

(d)

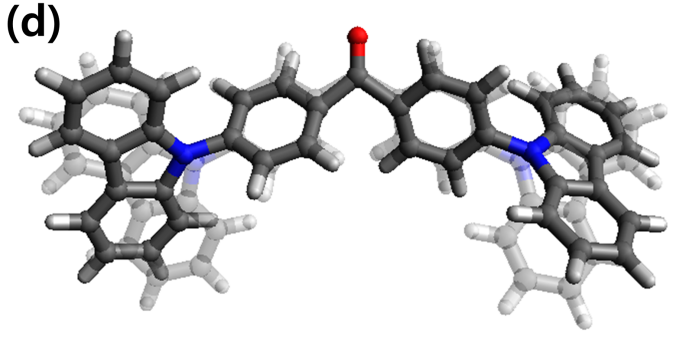

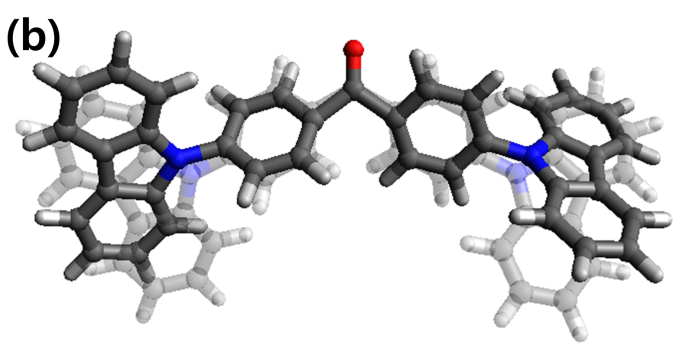

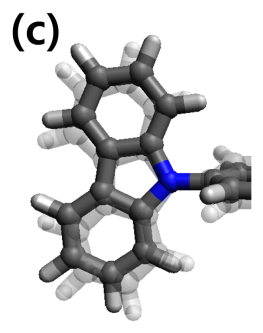

(e)

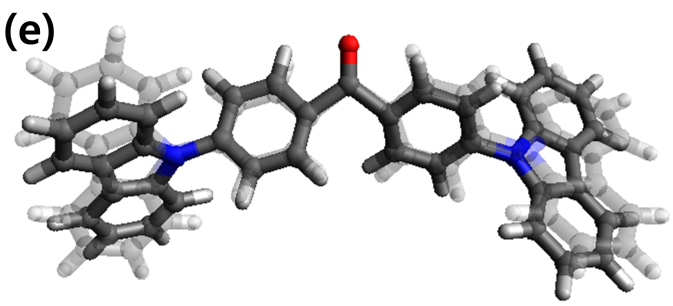

Figure S1: Geometries of $\mathbf{C z 2 B P}$ at (a) the energy minimum $(\Lambda=\infty)$; (b) the TADF maximum with no energy penalty $(\Lambda=0)$; (c) the donor-acceptor bond (maginfied view); $(\mathrm{d})$ the TADF maximum with energy penalty $\left(\Lambda=8.0 \mathrm{\mu s}^{-1} E_{\mathrm{h}}{ }^{-1}\right)$; and (e) the TADF maximum $(\Lambda=0)$ after the relaxation of the hydrogen atoms and the aromatic ring systems. The arrows in (a) indicate the DOFs that are the most active in the TADF rate maximization, and the translucent geometries in the background are the energy minimum in (b-d) and the TADF maximum with no energy penalty in (e).

The maximum TADF geometries of Cz2BP have been illustrated in Figure S1. Figure S1a shows the minimum energy conformation with arrows indicating the degrees of freedom (DOFs) that are the most active in the TADF rate maximization. Going to the maximum TADF conformation in Figure S1b, the carbazole ( $\mathrm{Cz}$, donor) and the phenyl groups in the benzophenone (BP, acceptor) group have rotated to increase the dihedral angles between the adjacent aromatic ring systems. The adjacent aromatic units are $73.7^{\circ}$ (left), $57.2^{\circ}$ (center), and $71.5^{\circ}$ (right) to each other, respectively, in contrast to $52.6^{\circ}$ (left), $52.9^{\circ}$ (center), and $52.8^{\circ}$ (right) at the energy minimum. While the increase in the DA dihedral angle is consistent with the fact that the ST gap and the TDM decrease, the benefit of increasing the dihedral angle between the phenyl rings in the acceptor group is not clear.

Meanwhile, the $\mathrm{Cz}$ and the phenyl groups also tilt in the direction of the carboxyl group 
so the overall molecule looks less bent in the maximum TADF rate conformation. In particular, the $\mathrm{Cz}$ group ends up with a gooseneck, as shown in Figures S1c. The differences in the $\mathrm{C}_{\mathrm{Cz}}-\mathrm{N}_{\mathrm{Cz}}-\mathrm{C}_{\mathrm{Ph}}$ angles at each carbazole group are $18.3^{\circ}$ (left) and $15.7^{\circ}$ (right), respectively, whereas the $\mathrm{C}_{\mathrm{Cz}}-\mathrm{N}_{\mathrm{Cz}}-\mathrm{C}_{\mathrm{Ph}}$ angles in the minimum energy conformation are the same $125.8^{\circ} \pm 0.1^{\circ}$. As shown in Figure S1d, the gooseneck persists even with an energy penalty of $\Lambda=8.0 \mathrm{\mu s}^{-1} E_{\mathrm{h}}^{-1}$, retaining $17.1^{\circ}$ (left) and $14.5^{\circ}$ (right) differences in the $\mathrm{C}_{\mathrm{Cz}}-\mathrm{N}_{\mathrm{Cz}}-\mathrm{C}_{\mathrm{Ph}}$ angles. Figure S1e shows the geometry after the hydrogen atoms and the aromatic ring systems have been relaxed. Visually, the relaxation of the aromatic units seems to somewhat undo the overall twist.

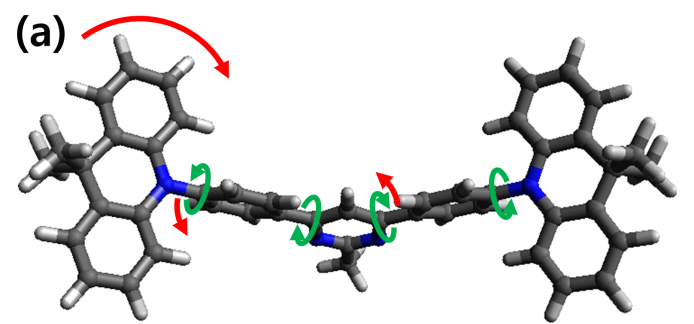

(b)
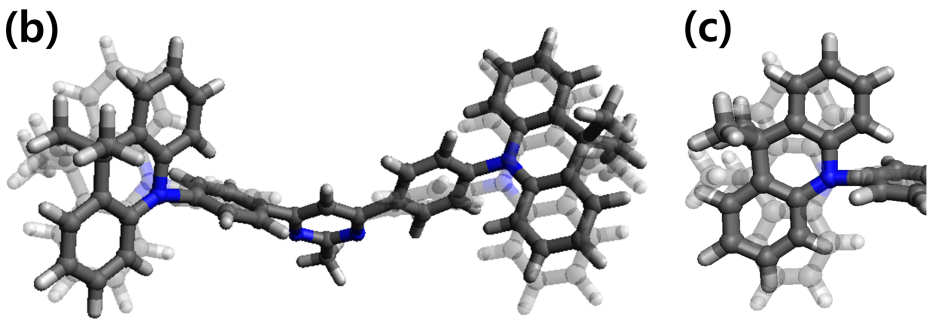

(d)

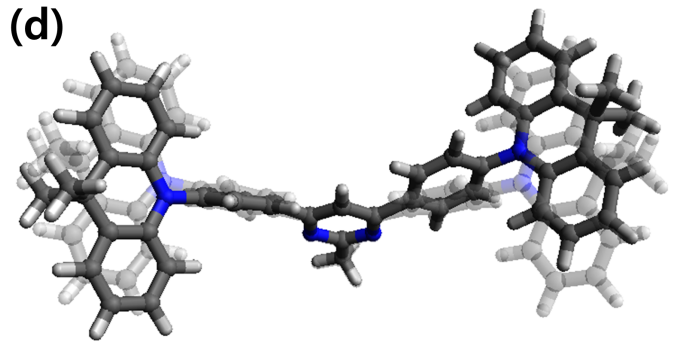

(e)

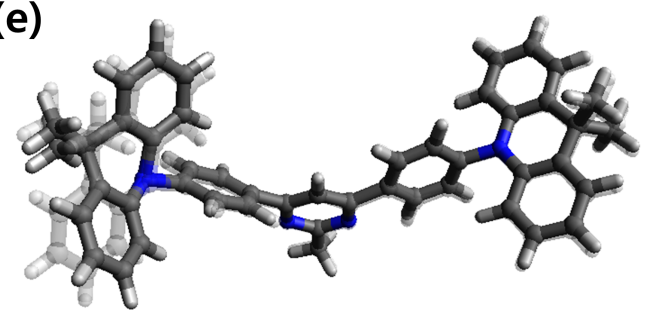

Figure S2: Geometries of Ac-MPM at (a) the energy minimum $(\Lambda=\infty)$; (b) the TADF maximum with no energy penalty $(\Lambda=0)$; (c) the donor-acceptor bond (maginfied view); (d) the TADF maximum with energy penalty $\left(\Lambda=8.0 \mu \mathrm{s}^{-1} E_{\mathrm{h}}{ }^{-1}\right)$; and (e) the TADF maximum $(\Lambda=0)$ after the relaxation of the hydrogen atoms and the aromatic ring systems. The arrows in (a) indicate the DOFs that are the most active in the TADF rate maximization, and the translucent geometries in the background are the energy minimum in (b-d) and the TADF maximum with no energy penalty in (e).

The maximum TADF geometries of Ac-MPM have been illustrated in Figure S2. Figure S2a shows the minimum energy conformation with arrows indicating the DOFs that are the most active in the TADF rate maximization. Unlike the cases of DCzTrz and $\mathbf{C z 2 B P}$, the dihedral angles between the donor and the acceptor groups decrease, consistent with the 
fact that the ST gap and the TDM increases in Ac-MPM. The acridine (Ac) groups are $75.2^{\circ}$ (left) and $74.8^{\circ}$ (right) to the phenyl rings of the 2-methylpyrimidine (MPM, acceptor) group, respectively, in contrast to $90.0^{\circ}$ (left) and $90.4^{\circ}$ (right) in the minimum energy conformation.

Nonetheless, familiar motifs appear in the maximum TADF rate conformation (Figure $\mathrm{S} 2 \mathrm{~b})$. One of the Ac groups tilts towards MPM group in a sharp gooseneck, as shown in Figure S2c. The differences in the $\mathrm{C}_{\mathrm{Ac}}-\mathrm{N}_{\mathrm{Ac}}-\mathrm{C}_{\mathrm{Ph}}$ angles at each acridine group are 55.0 (left) and $7.9^{\circ}$ (right), respectively, whereas the $\mathrm{C}_{\mathrm{Ac}}-\mathrm{N}_{\mathrm{Ac}}-\mathrm{C}_{\mathrm{Ph}}$ angles in the minimum energy conformation are identical within $119.2^{\circ} \pm 0.2^{\circ}$. In addition, the MPM group twists in an asymmetric manner. One of the phenyl rings twists out of plane. While the other phenyl ring also reorients itself, it maintains a moderate angle to the plane of the pyrimidine ring. The phenyl rings are $48.4^{\circ}$ (left) and $19.3^{\circ}$ (right) to the pyrimidine ring, respectively, in contrast to $18.7^{\circ}$ (left) and $17.7^{\circ}$ (right) in the minimum energy conformation.

As shown in Figure S2d, the twist in the acceptor group persists even with an energy penalty of $\Lambda=8.0 \mathrm{\mu s}^{-1} E_{\mathrm{h}}{ }^{-1}$, though the angles between the phenyl rings and the pyrimidine ring change to $30.0^{\circ}$ (left) and $17.2^{\circ}$ (right), respectively. However, the sharp gooseneck dissipates at some point between $4.0 \mathrm{\mu s}^{-1} E_{\mathrm{h}}{ }^{-1}$ and $8.0 \mathrm{\mu s}^{-1} E_{\mathrm{h}}{ }^{-1}$, the differences in the $\mathrm{C}_{\mathrm{Ac}^{-}}$ $\mathrm{N}_{\mathrm{Ac}}-\mathrm{C}_{\mathrm{Ph}}$ angles reduced to $5.0^{\circ}$ (left) and $4.2^{\circ}$ (right), respectively. We conjecture that the gooseneck provides negligible benefit to TADF in the case of Ac-MPM. Indeed, the twist in the acceptor group would skew the distribution of the LUMO towards the Ac group on the right, so the Ac group on the left would have limited impact on the TADF rate. Hence, it becomes favorable to unroll the gooseneck under even moderate energy penalties.

As shown in Figure S2(e), relaxing the hydrogen atoms and the aromatic ring systems puckers the Ac group on the left. In spite of the obvious disruption to the structure, the TADF rate only decreases by $16 \%$, in line with the conjecture that the Ac group on the right dominates the TADF process in the maximum TADF rate conformation of Ac-MPM.

The maximum TADF geometries of SpiroAC-TRZ have been illustrated in Figure S3. 

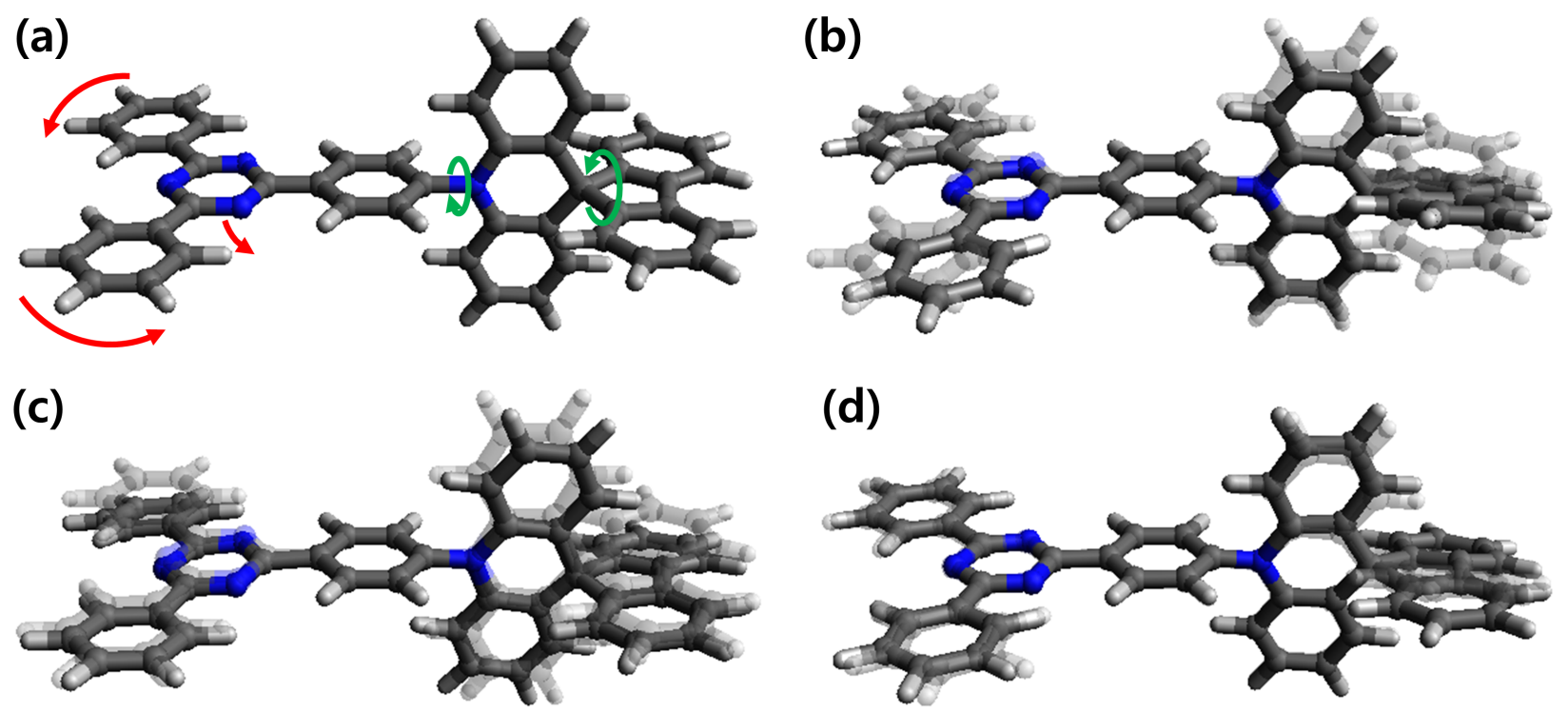

Figure S3: Geometries of SpiroAC-TRZ at (a) the energy minimum $(\Lambda=\infty)$; $(\mathrm{b})$ the TADF maximum with no energy penalty $(\Lambda=0)$; (c) the TADF maximum with energy penalty $\left(\Lambda=8.0 \mathrm{us}^{-1} E_{\mathrm{h}}{ }^{-1}\right)$; and $(\mathrm{d})$ the TADF maximum $(\Lambda=0)$ after the relaxation of the hydrogen atoms and the aromatic ring systems. The arrows in (a) indicate the DOFs that are the most active in the TADF rate maximization, and the translucent geometries in the background are the energy minimum in (b-c) and the TADF maximum with no energy penalty in (d).

Figure S3(a) shows the minimum energy conformation with arrows indicating the DOFs that are the most active in the TADF rate maximization. Once again, the dihedral angle between the donor group and the acceptor group decreases, consistent with the fact that the ST gap increases and the TDM decreases. The adjacent aromatic units are $83.9^{\circ}$ and $62.2^{\circ}$ to each other, respectively, in contrast to $90.1^{\circ}$ and $90.0^{\circ}$ in the minimum energy conformation. Unlike any of the previous cases, the maximum TADF rate conformation in Figure S3(b) shows neither a gooseneck nor a twist in the acceptor group. In fact, most of the distortion is contained in the wagging of the phenyl groups in the plane of the Trz group. As shown in Figure S3(d), relaxing the hydrogen atoms and the aromatic ring systems appears to restore the relative positions of the atoms in the minimum energy conformation. Given that the effect of the constrained energy minimization on the TADF rate is not severe, it must be the case that the relaxation does not affect the orbital overlap. Indeed, the dihedral angles between aromatic units have been constrained, and SpiroAC-TRZ does not contain 
a goose neck. Distortions within aromatic ring systems would have minimal effect on the orbital overlaps. 


\section{Natural Transition Orbitals}

The 3D images of the natural transition orbitals (NTOs) ${ }^{31}$ were generated in MacMolPlt $7.7 .^{32}$

(a)

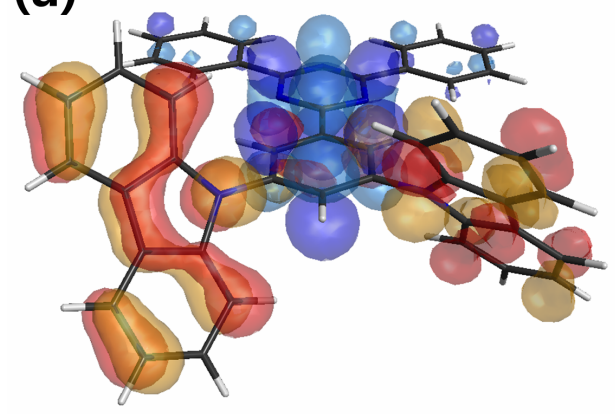

(c)

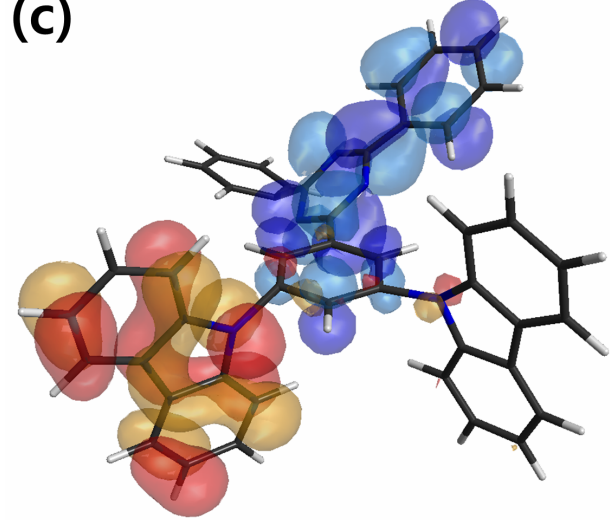

(b)

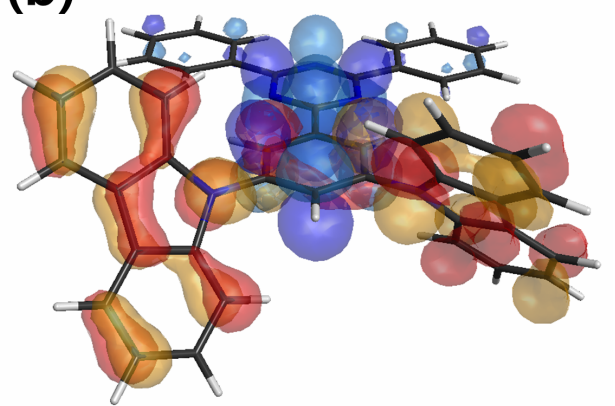

(d)

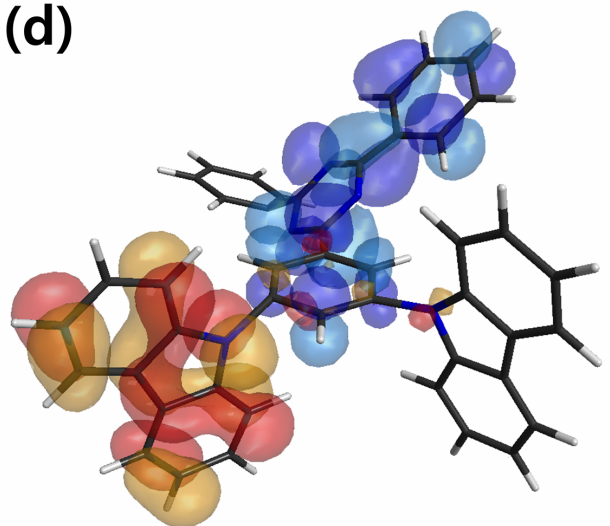

Figure S4: Dominant NTO pairs of DCzTrz in (a) the $\mathrm{S}_{1}$ state and (b) the $\mathrm{T}_{1}$ state at the energy minimum $(\Lambda=\infty)$; and $(\mathrm{c})$ the $\mathrm{S}_{1}$ state and $(\mathrm{d})$ the $\mathrm{T}_{1}$ state at the TADF maximum with no energy penalty $(\Lambda=0)$. The hole orbitals are shown in red and orange, and the electron orbitals are shown in sky blue and indigo. Contour value: 0.025 . 
(a)

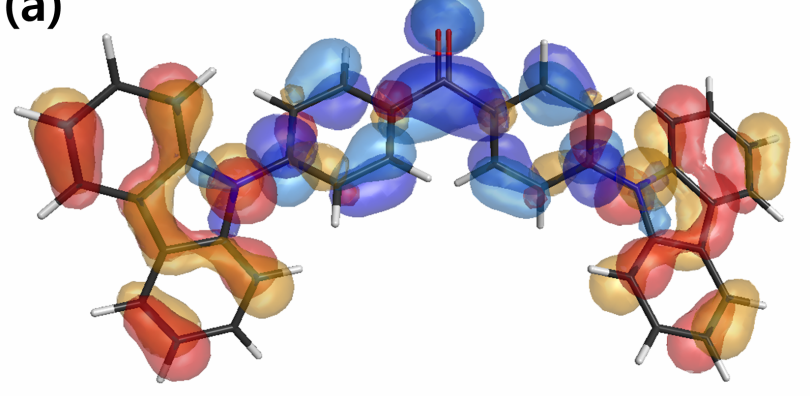

(c)

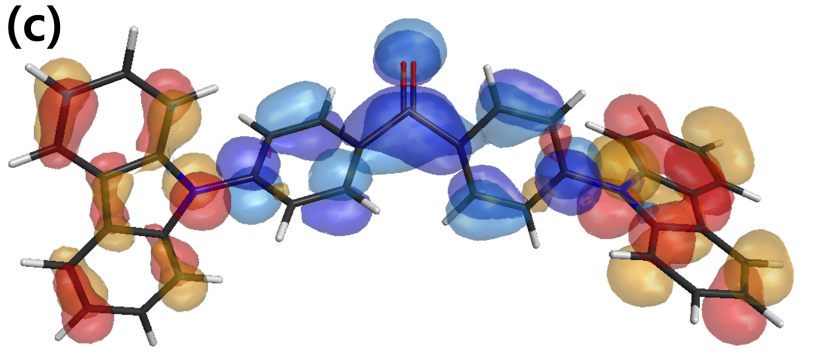

(b)

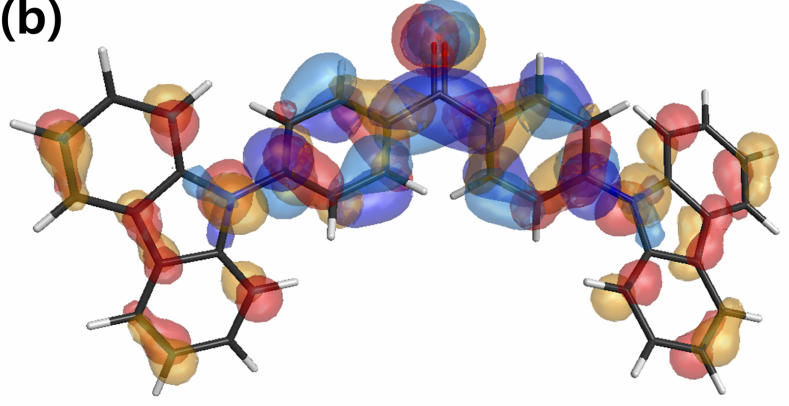

(d)

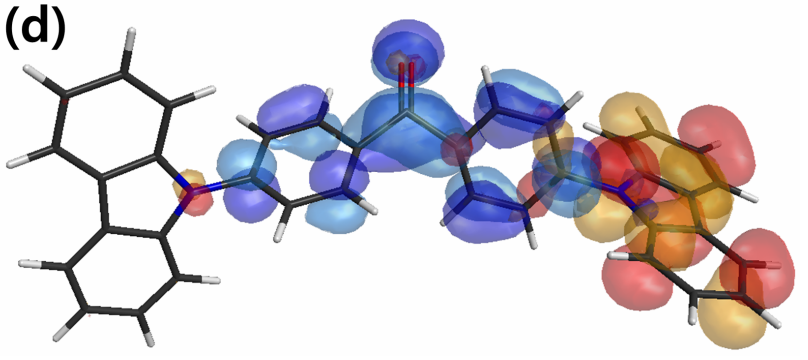

Figure S5: Dominant NTO pairs of Cz2BP in (a) the $\mathrm{S}_{1}$ state and (b) the $\mathrm{T}_{1}$ state at the energy minimum $(\Lambda=\infty)$; and (c) the $S_{1}$ state and (d) the $T_{1}$ state at the TADF maximum with no energy penalty $(\Lambda=0)$. The hole orbitals are shown in red and orange, and the electron orbitals are shown in sky blue and indigo. Contour value: 0.025.

(a)

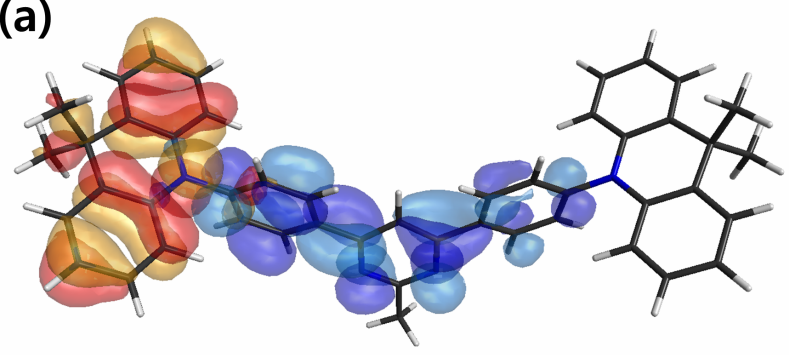

(c)

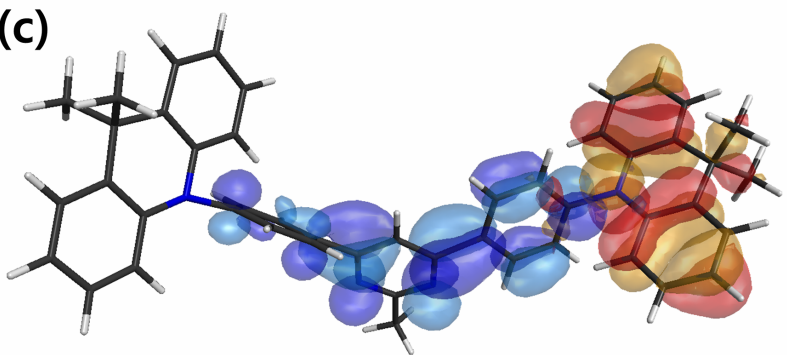

(b)

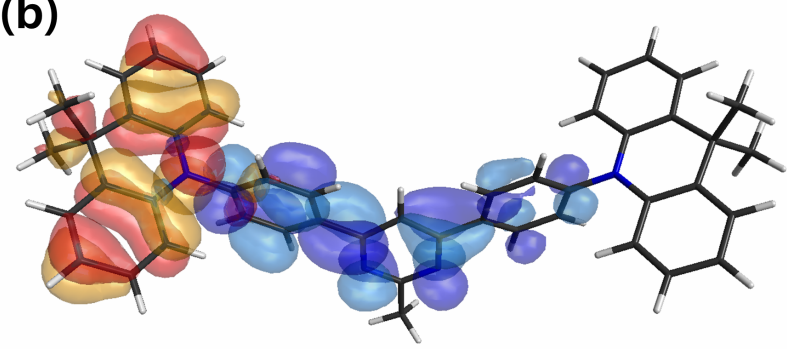

(d)

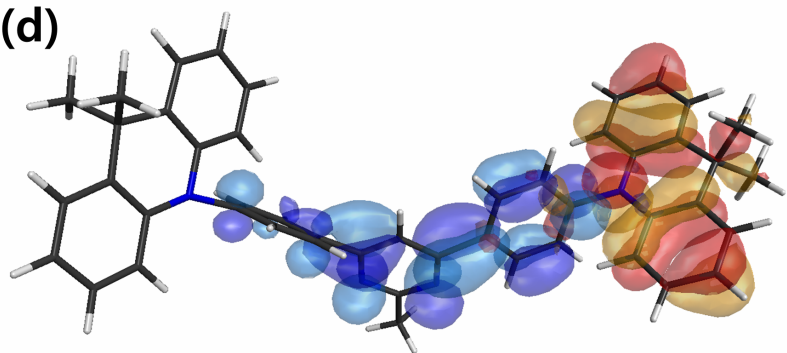

Figure S6: Dominant NTO pairs of Ac-MPM in (a) the $\mathrm{S}_{1}$ state and (b) the $\mathrm{T}_{1}$ state at the energy minimum $(\Lambda=\infty)$; and (c) the $S_{1}$ state and (d) the $T_{1}$ state at the TADF maximum with no energy penalty $(\Lambda=0)$. The hole orbitals are shown in red and orange, and the electron orbitals are shown in sky blue and indigo. Contour value: 0.025 . 
(a)

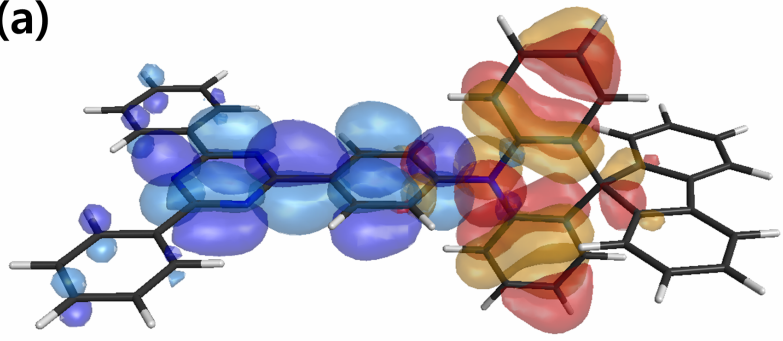

(c)

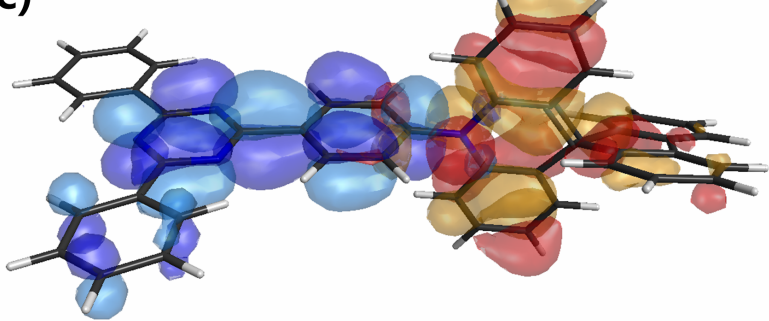

(b)

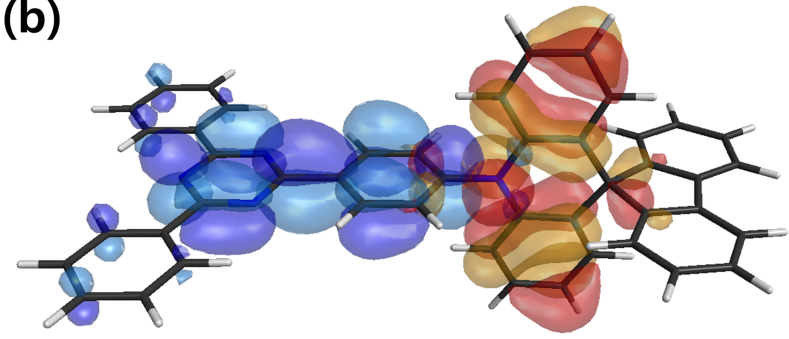

(d)

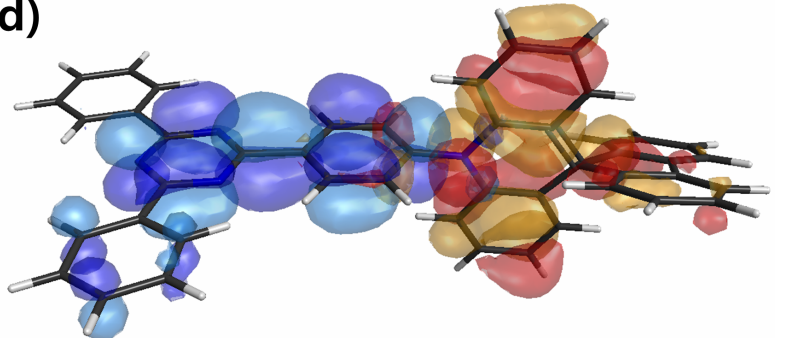

Figure S7: Dominant NTO pairs of SpiroAC-TRZ in (a) the $\mathrm{S}_{1}$ state and (b) the $\mathrm{T}_{1}$ state at the energy minimum $(\Lambda=\infty)$; and (c) the $S_{1}$ state and $(d)$ the $T_{1}$ state at the TADF maximum with no energy penalty $(\Lambda=0)$. The hole orbitals are shown in red and orange, and the electron orbitals are shown in sky blue and indigo. Contour value: 0.025. 


\section{Z-Matrices}

Tables S7-S28 show the Z-matrices of the model systems at the energy minimum, at the TADF maxima with various energy penalties, and after the relaxation of the hydrogen atoms and the aromatic ring systems. Note that Q-Chem reorders the Z-matrix during the constrained energy minimization.

Table S7: Z-matrix of DCzTrz at the energy minimum $(\Lambda=\infty)$.

\begin{tabular}{|c|c|c|c|c|c|c|c|c|c|c|c|c|c|}
\hline $\mathrm{C}$ & 1 & 1.39881 & & & & & & & & & & & \\
\hline $\mathrm{C}$ & 2 & 1.39425 & 1 & 120.16424 & & & & & & & & & \\
\hline $\mathrm{C}$ & 3 & 1.40529 & 2 & 120.33344 & 1 & 0.05219 & $\mathrm{C}$ & 41 & 1.40726 & 40 & 120.54922 & 39 & 359.65191 \\
\hline $\mathrm{C}$ & 4 & 1.40538 & 3 & 119.18851 & 2 & 359.95385 & $\mathrm{C}$ & 42 & 1.39528 & 41 & 121.40346 & 40 & 0.34748 \\
\hline $\mathrm{C}$ & 5 & 1.39442 & 4 & 120.32961 & 3 & 0.02562 & $\mathrm{C}$ & 43 & 1.39880 & 42 & 117.72197 & 41 & 0.21165 \\
\hline $\mathrm{C}$ & 4 & $1.48313^{*}$ & 3 & $120.37194^{*}$ & 2 & $179.97438^{*}$ & $\mathrm{C}$ & 9 & $1.48313^{*}$ & 8 & $118.15009^{*}$ & 7 & $180.02562^{*}$ \\
\hline $\mathrm{N}$ & 7 & 1.34191 & 4 & $118.12045^{*}$ & 3 & $1.04697^{*}$ & $\mathrm{C}$ & 45 & 1.40530 & 9 & $120.41287^{*}$ & 8 & $0.46503^{*}$ \\
\hline C & 8 & 1.34180 & 7 & 116.19320 & 4 & $179.97438^{*}$ & $\mathrm{C}$ & 46 & 1.39433 & 45 & 120.33164 & 9 & $179.97438^{*}$ \\
\hline $\mathrm{N}$ & 9 & 1.34395 & 8 & 123.87602 & 7 & 0.15279 & $\mathrm{C}$ & 47 & 1.39877 & 46 & 120.16975 & 45 & 0.02562 \\
\hline $\mathrm{C}$ & 10 & 1.33948 & 9 & 115.76541 & 8 & 359.79882 & $\mathrm{C}$ & 48 & 1.39863 & 47 & 119.81686 & 46 & 359.97438 \\
\hline $\mathrm{N}$ & 11 & 1.33944 & 10 & 124.53015 & 9 & 0.03803 & $\mathrm{C}$ & 49 & 1.39441 & 48 & 120.16153 & 47 & 359.97438 \\
\hline C & 11 & $1.48822^{*}$ & 10 & $117.70003^{*}$ & 9 & $180.02562^{*}$ & $\mathrm{H}$ & 1 & 1.08713 & 2 & 120.08954 & 3 & 179.97438 \\
\hline C & 13 & 1.40145 & 11 & $120.08922^{*}$ & 10 & $3.55655^{*}$ & $\mathrm{H}$ & 2 & 1.08691 & 1 & 120.09233 & 3 & 179.97438 \\
\hline $\mathrm{C}$ & 14 & 1.39772 & 13 & 120.10899 & 11 & $180.54975^{*}$ & $\mathrm{H}$ & 3 & 1.08429 & 2 & 120.63089 & 1 & 180.02562 \\
\hline $\mathrm{C}$ & 15 & 1.40041 & 14 & 120.13438 & 13 & 359.12975 & $\mathrm{H}$ & 5 & 1.08423 & 4 & 119.14092 & 3 & 180.02562 \\
\hline $\mathrm{C}$ & 16 & 1.40041 & 15 & 119.75732 & 14 & 0.36550 & $\mathrm{H}$ & 6 & 1.08684 & 5 & 119.70222 & 4 & 180.02562 \\
\hline C & 17 & 1.39763 & 16 & 120.17535 & 15 & 0.52083 & $\mathrm{H}$ & 14 & 1.08328 & 13 & 119.60739 & 18 & -178.97627 \\
\hline $\mathrm{N}$ & 17 & $1.42043^{*}$ & 16 & $119.84005^{*}$ & 15 & $179.97438^{*}$ & $\mathrm{H}$ & 16 & 1.08513 & 15 & 120.13739 & 14 & 180.33415 \\
\hline $\mathrm{C}$ & 19 & 1.40078 & 17 & $125.61232^{*}$ & 16 & $122.71268^{*}$ & $\mathrm{H}$ & 18 & 1.08335 & 17 & 120.27300 & 16 & 178.51517 \\
\hline C & 20 & 1.39884 & 19 & 129.44131 & 17 & $358.59290^{*}$ & $\mathrm{H}$ & 21 & 1.08557 & 20 & 121.49918 & 19 & 1.06266 \\
\hline $\mathrm{C}$ & 21 & 1.39526 & 20 & 117.71657 & 19 & 181.18901 & $\mathrm{H}$ & 22 & 1.08691 & 21 & 119.07827 & 20 & 180.02562 \\
\hline $\mathrm{C}$ & 22 & 1.40729 & 21 & 121.40581 & 20 & 0.17108 & $\mathrm{H}$ & 23 & 1.08658 & 22 & 119.59854 & 21 & 180.02562 \\
\hline $\mathrm{C}$ & 23 & 1.39379 & 22 & 120.54855 & 21 & 0.35645 & $\mathrm{H}$ & 24 & 1.08723 & 23 & 120.41591 & 22 & 179.42469 \\
\hline $\mathrm{C}$ & 24 & 1.40103 & 23 & 119.18251 & 22 & 359.67902 & $\mathrm{H}$ & 27 & 1.08723 & 26 & 120.40812 & 25 & 359.13361 \\
\hline $\mathrm{C}$ & 25 & 1.44891 & 24 & 133.61296 & 23 & 178.87145 & $\mathrm{H}$ & 28 & 1.08654 & 27 & 119.85378 & 26 & 179.90485 \\
\hline $\mathrm{C}$ & 26 & 1.40095 & 25 & 133.59767 & 24 & 1.68791 & $\mathrm{H}$ & 29 & 1.08685 & 28 & 119.52324 & 27 & 180.49587 \\
\hline $\mathrm{C}$ & 27 & 1.39372 & 26 & 119.17823 & 25 & 178.91015 & $\mathrm{H}$ & 30 & 1.08561 & 29 & 120.75101 & 28 & 180.51128 \\
\hline $\mathrm{C}$ & 28 & 1.40722 & 27 & 120.55061 & 26 & 359.62121 & $\mathrm{H}$ & 34 & 1.08554 & 33 & 121.53615 & 32 & 0.80805 \\
\hline C & 29 & 1.39519 & 28 & 121.40591 & 27 & 0.41068 & $\mathrm{H}$ & 35 & 1.08685 & 34 & 119.07160 & 33 & 180.09013 \\
\hline C & 30 & 1.39882 & 29 & 117.72369 & 28 & 0.19095 & $\mathrm{H}$ & 36 & 1.08654 & 35 & 119.59660 & 34 & 180.12953 \\
\hline $\mathrm{N}$ & 15 & $1.42021^{*}$ & 14 & $119.96093^{*}$ & 13 & $179.64405^{*}$ & $\mathrm{H}$ & 37 & 1.08722 & 36 & 120.42035 & 35 & 179.37317 \\
\hline C & 32 & 1.40113 & 15 & $125.95848^{*}$ & 14 & $122.96261^{*}$ & $\mathrm{H}$ & 40 & 1.08723 & 39 & 120.40225 & 38 & 359.28562 \\
\hline $\mathrm{C}$ & 33 & 1.39881 & 32 & 129.47780 & 15 & $358.32390^{*}$ & $\mathrm{H}$ & 41 & 1.08658 & 40 & 119.85209 & 39 & 179.97438 \\
\hline $\mathrm{C}$ & 34 & 1.39520 & 33 & 117.72493 & 32 & 181.18158 & $\mathrm{H}$ & 42 & 1.08690 & 41 & 119.52479 & 40 & 180.44541 \\
\hline $\mathrm{C}$ & 35 & 1.40718 & 34 & 121.40971 & 33 & 0.18689 & $\mathrm{H}$ & 43 & 1.08550 & 42 & 120.77406 & 41 & 180.30187 \\
\hline C & 36 & 1.39370 & 35 & 120.54803 & 34 & 0.41641 & $\mathrm{H}$ & 46 & 1.08430 & 45 & 119.05000 & 50 & -179.97489 \\
\hline $\mathrm{C}$ & 37 & 1.40094 & 36 & 119.17491 & 35 & 359.62614 & $\mathrm{H}$ & 47 & 1.08692 & 46 & 119.73749 & 45 & 180.02562 \\
\hline $\mathrm{C}$ & 38 & 1.44896 & 37 & 133.57589 & 36 & 178.74217 & $\mathrm{H}$ & 48 & 1.08712 & 47 & 120.08957 & 46 & 180.02562 \\
\hline $\mathrm{C}$ & 39 & 1.40108 & 38 & 133.61407 & 37 & 1.67108 & $\mathrm{H}$ & 49 & 1.08684 & 48 & 120.13668 & 47 & 179.97438 \\
\hline $\mathrm{C}$ & 40 & 1.39378 & 39 & 119.17892 & 38 & 179.07456 & $\mathrm{H}$ & 50 & 1.08420 & 49 & 120.52043 & 48 & 179.97438 \\
\hline
\end{tabular}


Table S8: Z-matrix of DCzTrz at the TADF maximum with energy penalty $(\Lambda=$ $\left.8.0 \mathrm{\mu s}^{-1} E_{\mathrm{h}}{ }^{-1}\right)$.

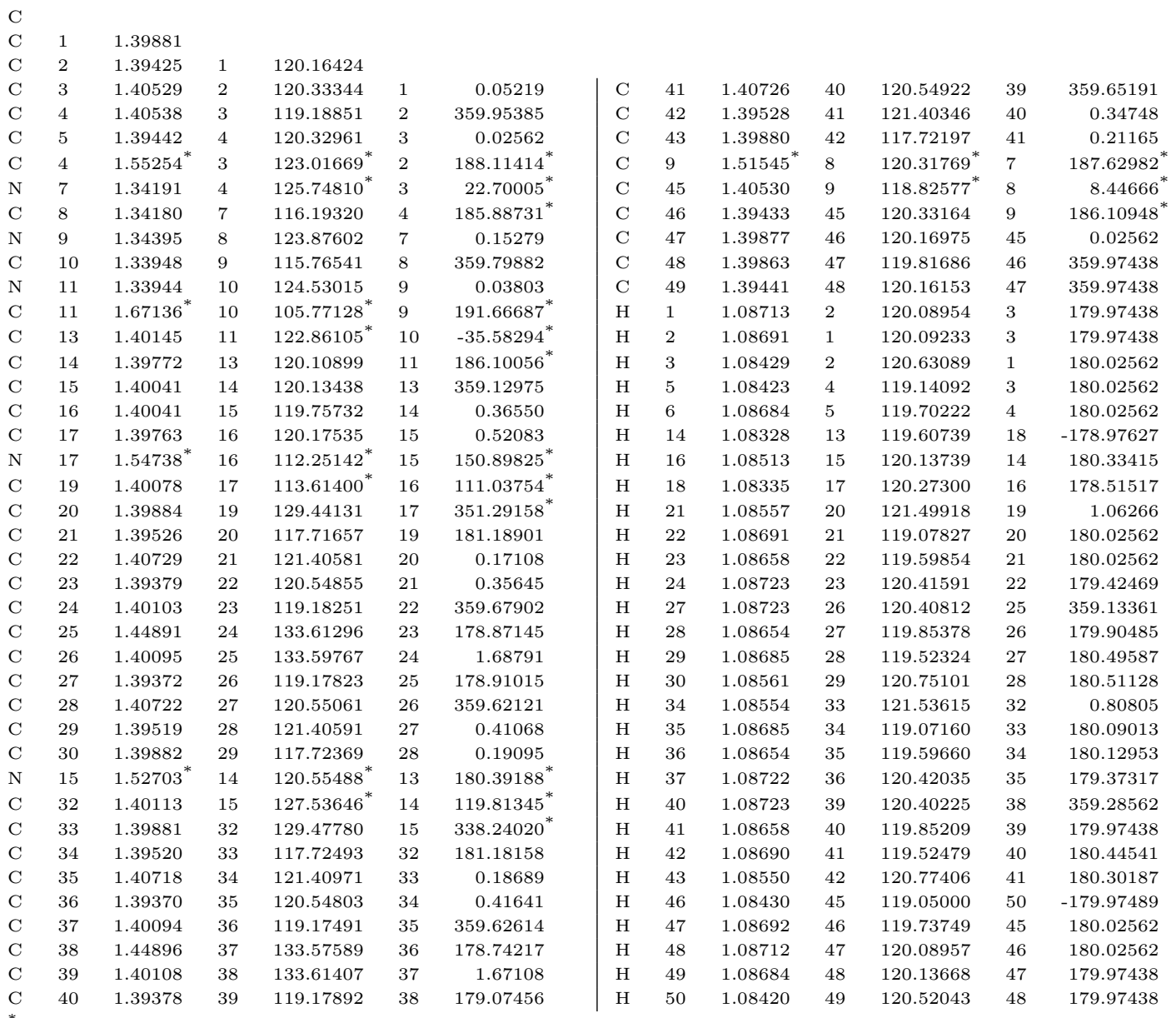


Table S9: Z-matrix of DCzTrz at the TADF maximum with energy penalty $(\Lambda=$ $4.0 \mathrm{\mu s}^{-1} E_{\mathrm{h}}^{-1}$ ).

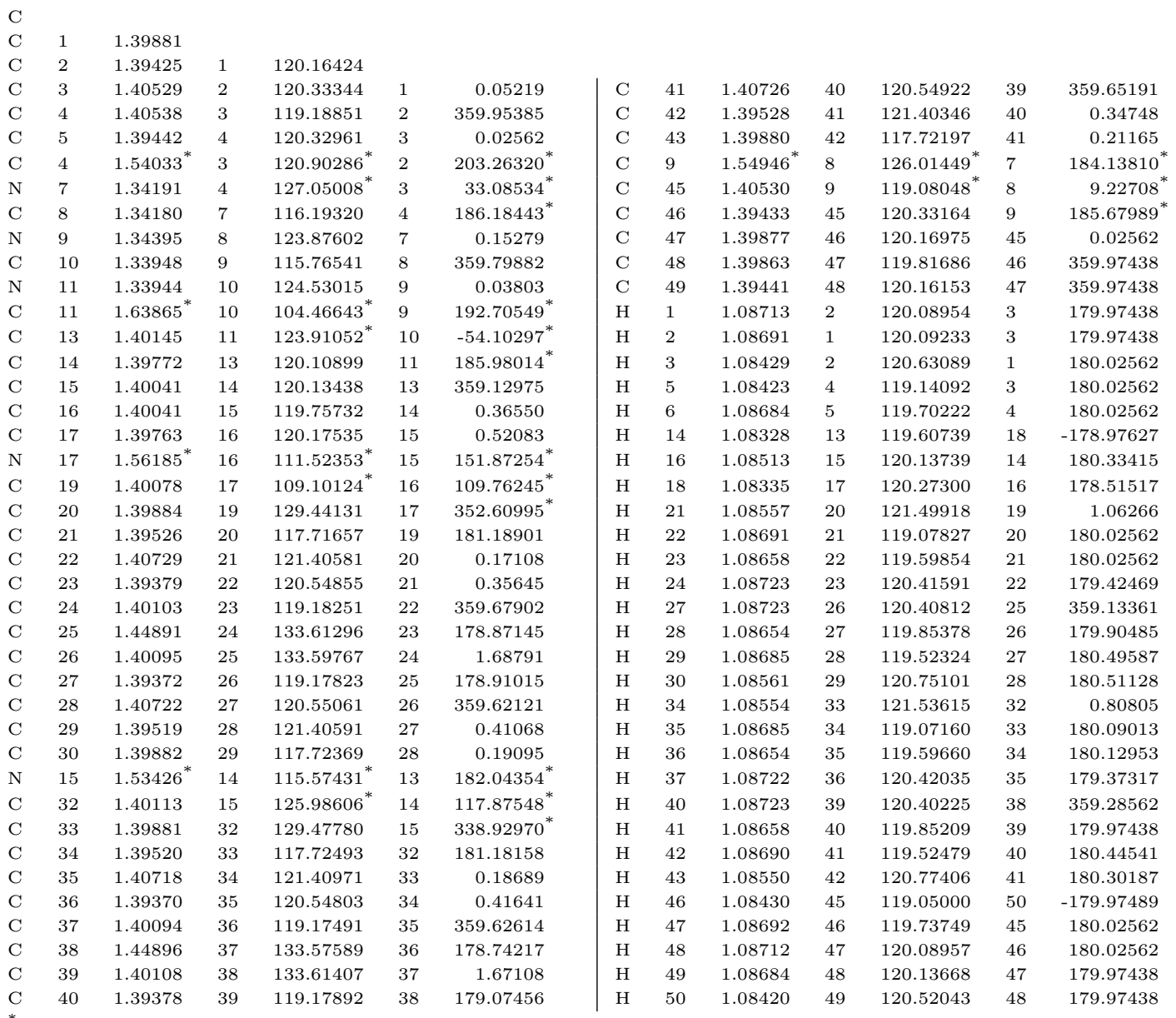


Table S10: Z-matrix of DCzTrz at the TADF maximum with energy penalty $(\Lambda=$ $\left.2.0 \mathrm{\mu s}^{-1} E_{\mathrm{h}}^{-1}\right)$.

\begin{tabular}{|c|c|c|c|c|c|c|c|c|c|c|c|c|c|}
\hline $\mathrm{C}$ & 1 & 1.39881 & & & & & & & & & & & \\
\hline $\mathrm{C}$ & 2 & 1.39425 & 1 & 120.16424 & & & & & & & & & \\
\hline $\mathrm{C}$ & 3 & 1.40529 & 2 & 120.33344 & 1 & 0.05219 & $\mathrm{C}$ & 41 & 1.40726 & 40 & 120.54922 & 39 & 359.65191 \\
\hline $\mathrm{C}$ & 4 & 1.40538 & 3 & 119.18851 & 2 & 359.95385 & $\mathrm{C}$ & 42 & 1.39528 & 41 & 121.40346 & 40 & 0.34748 \\
\hline C & 5 & 1.39442 & 4 & 120.32961 & 3 & 0.02562 & $\mathrm{C}$ & 43 & 1.39880 & 42 & 117.72197 & 41 & 0.21165 \\
\hline C & 4 & $1.54711^{*}$ & 3 & $130.32246^{*}$ & 2 & $216.04060^{*}$ & $\mathrm{C}$ & 9 & $1.44082^{*}$ & 8 & $130.39469^{*}$ & 7 & $185.92006^{*}$ \\
\hline $\mathrm{N}$ & 7 & 1.34191 & 4 & $125.93354^{*}$ & 3 & $29.60538^{*}$ & $\mathrm{C}$ & 45 & 1.40530 & 9 & $104.38526^{*}$ & 8 & $8.43189^{*}$ \\
\hline $\mathrm{C}$ & 8 & 1.34180 & 7 & 116.19320 & 4 & $187.01069^{*}$ & $\mathrm{C}$ & 46 & 1.39433 & 45 & 120.33164 & 9 & $188.28194^{*}$ \\
\hline $\mathrm{N}$ & 9 & 1.34395 & 8 & 123.87602 & 7 & 0.15279 & $\mathrm{C}$ & 47 & 1.39877 & 46 & 120.16975 & 45 & 0.02562 \\
\hline $\mathrm{C}$ & 10 & 1.33948 & 9 & 115.76541 & 8 & 359.79882 & $\mathrm{C}$ & 48 & 1.39863 & 47 & 119.81686 & 46 & 359.97438 \\
\hline $\mathrm{N}$ & 11 & 1.33944 & 10 & 124.53015 & 9 & 0.03803 & $\mathrm{C}$ & 49 & 1.39441 & 48 & 120.16153 & 47 & 359.97438 \\
\hline C & 11 & $1.67937^{*}$ & 10 & $100.40804^{*}$ & 9 & $191.87496^{*}$ & $\mathrm{H}$ & 1 & 1.08713 & 2 & 120.08954 & 3 & 179.97438 \\
\hline C & 13 & 1.40145 & 11 & $123.28155^{*}$ & 10 & $-52.66865^{*}$ & $\mathrm{H}$ & 2 & 1.08691 & 1 & 120.09233 & 3 & 179.97438 \\
\hline C & 14 & 1.39772 & 13 & 120.10899 & 11 & $186.79199^{*}$ & $\mathrm{H}$ & 3 & 1.08429 & 2 & 120.63089 & 1 & 180.02562 \\
\hline C & 15 & 1.40041 & 14 & 120.13438 & 13 & 359.12975 & $\mathrm{H}$ & 5 & 1.08423 & 4 & 119.14092 & 3 & 180.02562 \\
\hline $\mathrm{C}$ & 16 & 1.40041 & 15 & 119.75732 & 14 & 0.36550 & $\mathrm{H}$ & 6 & 1.08684 & 5 & 119.70222 & 4 & 180.02562 \\
\hline $\mathrm{C}$ & 17 & 1.39763 & 16 & 120.17535 & 15 & 0.52083 & $\mathrm{H}$ & 14 & 1.08328 & 13 & 119.60739 & 18 & -178.97627 \\
\hline $\mathrm{N}$ & 17 & $1.53919^{*}$ & 16 & $109.12161^{*}$ & 15 & $145.98502^{*}$ & $\mathrm{H}$ & 16 & 1.08513 & 15 & 120.13739 & 14 & 180.33415 \\
\hline $\mathrm{C}$ & 19 & 1.40078 & 17 & $115.10068^{*}$ & 16 & $110.02287^{*}$ & $\mathrm{H}$ & 18 & 1.08335 & 17 & 120.27300 & 16 & 178.51517 \\
\hline C & 20 & 1.39884 & 19 & 129.44131 & 17 & $353.40779^{*}$ & $\mathrm{H}$ & 21 & 1.08557 & 20 & 121.49918 & 19 & 1.06266 \\
\hline $\mathrm{C}$ & 21 & 1.39526 & 20 & 117.71657 & 19 & 181.18901 & $\mathrm{H}$ & 22 & 1.08691 & 21 & 119.07827 & 20 & 180.02562 \\
\hline $\mathrm{C}$ & 22 & 1.40729 & 21 & 121.40581 & 20 & 0.17108 & $\mathrm{H}$ & 23 & 1.08658 & 22 & 119.59854 & 21 & 180.02562 \\
\hline C & 23 & 1.39379 & 22 & 120.54855 & 21 & 0.35645 & $\mathrm{H}$ & 24 & 1.08723 & 23 & 120.41591 & 22 & 179.42469 \\
\hline $\mathrm{C}$ & 24 & 1.40103 & 23 & 119.18251 & 22 & 359.67902 & $\mathrm{H}$ & 27 & 1.08723 & 26 & 120.40812 & 25 & 359.13361 \\
\hline $\mathrm{C}$ & 25 & 1.44891 & 24 & 133.61296 & 23 & 178.87145 & $\mathrm{H}$ & 28 & 1.08654 & 27 & 119.85378 & 26 & 179.90485 \\
\hline $\mathrm{C}$ & 26 & 1.40095 & 25 & 133.59767 & 24 & 1.68791 & $\mathrm{H}$ & 29 & 1.08685 & 28 & 119.52324 & 27 & 180.49587 \\
\hline $\mathrm{C}$ & 27 & 1.39372 & 26 & 119.17823 & 25 & 178.91015 & $\mathrm{H}$ & 30 & 1.08561 & 29 & 120.75101 & 28 & 180.51128 \\
\hline C & 28 & 1.40722 & 27 & 120.55061 & 26 & 359.62121 & $\mathrm{H}$ & 34 & 1.08554 & 33 & 121.53615 & 32 & 0.80805 \\
\hline $\mathrm{C}$ & 29 & 1.39519 & 28 & 121.40591 & 27 & 0.41068 & $\mathrm{H}$ & 35 & 1.08685 & 34 & 119.07160 & 33 & 180.09013 \\
\hline $\mathrm{C}$ & 30 & 1.39882 & 29 & 117.72369 & 28 & 0.19095 & $\mathrm{H}$ & 36 & 1.08654 & 35 & 119.59660 & 34 & 180.12953 \\
\hline $\mathrm{N}$ & 15 & $1.53780^{*}$ & 14 & $116.90182^{*}$ & 13 & $183.59926^{*}$ & $\mathrm{H}$ & 37 & 1.08722 & 36 & 120.42035 & 35 & 179.37317 \\
\hline C & 32 & 1.40113 & 15 & $128.71968^{*}$ & 14 & $115.09179^{*}$ & $\mathrm{H}$ & 40 & 1.08723 & 39 & 120.40225 & 38 & 359.28562 \\
\hline C & 33 & 1.39881 & 32 & 129.47780 & 15 & $334.79222^{*}$ & $\mathrm{H}$ & 41 & 1.08658 & 40 & 119.85209 & 39 & 179.97438 \\
\hline $\mathrm{C}$ & 34 & 1.39520 & 33 & 117.72493 & 32 & 181.18158 & $\mathrm{H}$ & 42 & 1.08690 & 41 & 119.52479 & 40 & 180.44541 \\
\hline C & 35 & 1.40718 & 34 & 121.40971 & 33 & 0.18689 & $\mathrm{H}$ & 43 & 1.08550 & 42 & 120.77406 & 41 & 180.30187 \\
\hline $\mathrm{C}$ & 36 & 1.39370 & 35 & 120.54803 & 34 & 0.41641 & $\mathrm{H}$ & 46 & 1.08430 & 45 & 119.05000 & 50 & -179.97489 \\
\hline $\mathrm{C}$ & 37 & 1.40094 & 36 & 119.17491 & 35 & 359.62614 & $\mathrm{H}$ & 47 & 1.08692 & 46 & 119.73749 & 45 & 180.02562 \\
\hline C & 38 & 1.44896 & 37 & 133.57589 & 36 & 178.74217 & $\mathrm{H}$ & 48 & 1.08712 & 47 & 120.08957 & 46 & 180.02562 \\
\hline $\mathrm{C}$ & 39 & 1.40108 & 38 & 133.61407 & 37 & 1.67108 & $\mathrm{H}$ & 49 & 1.08684 & 48 & 120.13668 & 47 & 179.97438 \\
\hline $\mathrm{C}$ & 40 & 1.39378 & 39 & 119.17892 & 38 & 179.07456 & $\mathrm{H}$ & 50 & 1.08420 & 49 & 120.52043 & 48 & 179.97438 \\
\hline
\end{tabular}


Table S11: Z-matrix of DCzTrz at the TADF maximum with energy penalty $(\Lambda=$

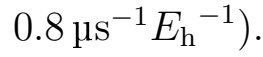

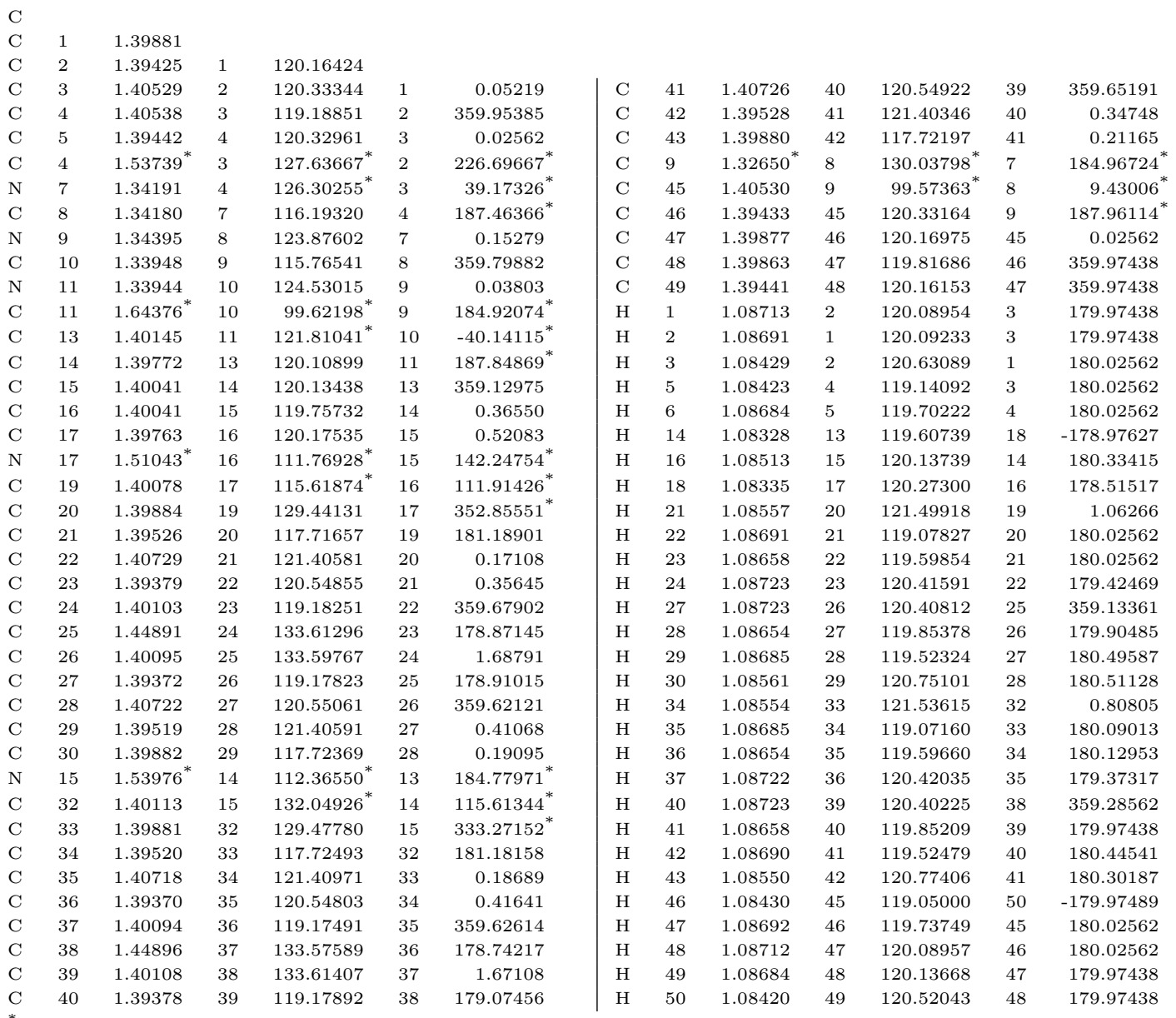


Table S12: Z-matrix of DCzTrz at the TADF maximum with no energy penalty $(\Lambda=0)$.

\begin{tabular}{|c|c|c|c|c|c|c|c|c|c|c|c|c|c|}
\hline $\mathrm{C}$ & & & & & & & & & & & & & \\
\hline $\mathrm{C}$ & 1 & 1.39881 & & & & & & & & & & & \\
\hline $\mathrm{C}$ & 2 & 1.39425 & 1 & 120.16424 & & & & & & & & & \\
\hline $\mathrm{C}$ & 3 & 1.40529 & 2 & 120.33344 & 1 & 0.05219 & $\mathrm{C}$ & 41 & 1.40726 & 40 & 120.54922 & 39 & 359.65191 \\
\hline $\mathrm{C}$ & 4 & 1.40538 & 3 & 119.18851 & 2 & 359.95385 & $\mathrm{C}$ & 42 & 1.39528 & 41 & 121.40346 & 40 & 0.34748 \\
\hline $\mathrm{C}$ & 5 & 1.39442 & 4 & 120.32961 & 3 & 0.02562 & $\mathrm{C}$ & 43 & 1.39880 & 42 & 117.72197 & 41 & 0.21165 \\
\hline $\mathrm{C}$ & 4 & $1.54804^{*}$ & 3 & $121.94919^{*}$ & 2 & $225.88712^{*}$ & $\mathrm{C}$ & 9 & $1.35542^{*}$ & 8 & $138.93351^{*}$ & 7 & $183.91568^{*}$ \\
\hline $\mathrm{N}$ & 7 & 1.34191 & 4 & $125.80883^{*}$ & 3 & $25.06701^{*}$ & $\mathrm{C}$ & 45 & 1.40530 & 9 & $98.66481^{*}$ & 8 & $9.05117^{*}$ \\
\hline C & 8 & 1.34180 & 7 & 116.19320 & 4 & $187.65876^{*}$ & $\mathrm{C}$ & 46 & 1.39433 & 45 & 120.33164 & 9 & $188.22049^{*}$ \\
\hline $\mathrm{N}$ & 9 & 1.34395 & 8 & 123.87602 & 7 & 0.15279 & $\mathrm{C}$ & 47 & 1.39877 & 46 & 120.16975 & 45 & 0.02562 \\
\hline C & 10 & 1.33948 & 9 & 115.76541 & 8 & 359.79882 & $\mathrm{C}$ & 48 & 1.39863 & 47 & 119.81686 & 46 & 359.97438 \\
\hline $\mathrm{N}$ & 11 & 1.33944 & 10 & 124.53015 & 9 & 0.03803 & $\mathrm{C}$ & 49 & 1.39441 & 48 & 120.16153 & 47 & 359.97438 \\
\hline $\mathrm{C}$ & 11 & $1.68377^{*}$ & 10 & $98.08918^{*}$ & 9 & $192.76451^{*}$ & $\mathrm{H}$ & 1 & 1.08713 & 2 & 120.08954 & 3 & 179.97438 \\
\hline C & 13 & 1.40145 & 11 & $123.00369^{*}$ & 10 & $-47.05700^{*}$ & $\mathrm{H}$ & 2 & 1.08691 & 1 & 120.09233 & 3 & 179.97438 \\
\hline $\mathrm{C}$ & 14 & 1.39772 & 13 & 120.10899 & 11 & $187.90564^{*}$ & $\mathrm{H}$ & 3 & 1.08429 & 2 & 120.63089 & 1 & 180.02562 \\
\hline $\mathrm{C}$ & 15 & 1.40041 & 14 & 120.13438 & 13 & 359.12975 & $\mathrm{H}$ & 5 & 1.08423 & 4 & 119.14092 & 3 & 180.02562 \\
\hline C & 16 & 1.40041 & 15 & 119.75732 & 14 & 0.36550 & $\mathrm{H}$ & 6 & 1.08684 & 5 & 119.70222 & 4 & 180.02562 \\
\hline C & 17 & 1.39763 & 16 & 120.17535 & 15 & 0.52083 & $\mathrm{H}$ & 14 & 1.08328 & 13 & 119.60739 & 18 & -178.97627 \\
\hline $\mathrm{N}$ & 17 & $1.55824^{*}$ & 16 & $113.04495^{*}$ & 15 & $143.34604^{*}$ & $\mathrm{H}$ & 16 & 1.08513 & 15 & 120.13739 & 14 & 180.33415 \\
\hline $\mathrm{C}$ & 19 & 1.40078 & 17 & $116.70497^{*}$ & 16 & $109.81953^{*}$ & $\mathrm{H}$ & 18 & 1.08335 & 17 & 120.27300 & 16 & 178.51517 \\
\hline C & 20 & 1.39884 & 19 & 129.44131 & 17 & $351.89001^{*}$ & $\mathrm{H}$ & 21 & 1.08557 & 20 & 121.49918 & 19 & 1.06266 \\
\hline $\mathrm{C}$ & 21 & 1.39526 & 20 & 117.71657 & 19 & 181.18901 & $\mathrm{H}$ & 22 & 1.08691 & 21 & 119.07827 & 20 & 180.02562 \\
\hline $\mathrm{C}$ & 22 & 1.40729 & 21 & 121.40581 & 20 & 0.17108 & $\mathrm{H}$ & 23 & 1.08658 & 22 & 119.59854 & 21 & 180.02562 \\
\hline $\mathrm{C}$ & 23 & 1.39379 & 22 & 120.54855 & 21 & 0.35645 & $\mathrm{H}$ & 24 & 1.08723 & 23 & 120.41591 & 22 & 179.42469 \\
\hline C & 24 & 1.40103 & 23 & 119.18251 & 22 & 359.67902 & $\mathrm{H}$ & 27 & 1.08723 & 26 & 120.40812 & 25 & 359.13361 \\
\hline C & 25 & 1.44891 & 24 & 133.61296 & 23 & 178.87145 & $\mathrm{H}$ & 28 & 1.08654 & 27 & 119.85378 & 26 & 179.90485 \\
\hline C & 26 & 1.40095 & 25 & 133.59767 & 24 & 1.68791 & $\mathrm{H}$ & 29 & 1.08685 & 28 & 119.52324 & 27 & 180.49587 \\
\hline C & 27 & 1.39372 & 26 & 119.17823 & 25 & 178.91015 & $\mathrm{H}$ & 30 & 1.08561 & 29 & 120.75101 & 28 & 180.51128 \\
\hline $\mathrm{C}$ & 28 & 1.40722 & 27 & 120.55061 & 26 & 359.62121 & $\mathrm{H}$ & 34 & 1.08554 & 33 & 121.53615 & 32 & 0.80805 \\
\hline C & 29 & 1.39519 & 28 & 121.40591 & 27 & 0.41068 & $\mathrm{H}$ & 35 & 1.08685 & 34 & 119.07160 & 33 & 180.09013 \\
\hline C & 30 & 1.39882 & 29 & 117.72369 & 28 & 0.19095 & $\mathrm{H}$ & 36 & 1.08654 & 35 & 119.59660 & 34 & 180.12953 \\
\hline $\mathrm{N}$ & 15 & $1.54035^{*}$ & 14 & $112.75538^{*}$ & 13 & $183.54298^{*}$ & $\mathrm{H}$ & 37 & 1.08722 & 36 & 120.42035 & 35 & 179.37317 \\
\hline C & 32 & 1.40113 & 15 & $133.50170^{*}$ & 14 & $115.24717^{*}$ & $\mathrm{H}$ & 40 & 1.08723 & 39 & 120.40225 & 38 & 359.28562 \\
\hline $\mathrm{C}$ & 33 & 1.39881 & 32 & 129.47780 & 15 & $333.24938^{*}$ & $\mathrm{H}$ & 41 & 1.08658 & 40 & 119.85209 & 39 & 179.97438 \\
\hline $\mathrm{C}$ & 34 & 1.39520 & 33 & 117.72493 & 32 & 181.18158 & $\mathrm{H}$ & 42 & 1.08690 & 41 & 119.52479 & 40 & 180.44541 \\
\hline C & 35 & 1.40718 & 34 & 121.40971 & 33 & 0.18689 & $\mathrm{H}$ & 43 & 1.08550 & 42 & 120.77406 & 41 & 180.30187 \\
\hline $\mathrm{C}$ & 36 & 1.39370 & 35 & 120.54803 & 34 & 0.41641 & $\mathrm{H}$ & 46 & 1.08430 & 45 & 119.05000 & 50 & -179.97489 \\
\hline C & 37 & 1.40094 & 36 & 119.17491 & 35 & 359.62614 & $\mathrm{H}$ & 47 & 1.08692 & 46 & 119.73749 & 45 & 180.02562 \\
\hline $\mathrm{C}$ & 38 & 1.44896 & 37 & 133.57589 & 36 & 178.74217 & $\mathrm{H}$ & 48 & 1.08712 & 47 & 120.08957 & 46 & 180.02562 \\
\hline C & 39 & 1.40108 & 38 & 133.61407 & 37 & 1.67108 & $\mathrm{H}$ & 49 & 1.08684 & 48 & 120.13668 & 47 & 179.97438 \\
\hline $\mathrm{C}$ & 40 & 1.39378 & 39 & 119.17892 & 38 & 179.07456 & $\mathrm{H}$ & 50 & 1.08420 & 49 & 120.52043 & 48 & 179.97438 \\
\hline
\end{tabular}


Table S13: Z-matrix of DCzTrz at the TADF maximum $(\Lambda=0)$ after the relaxation of the hydrogen atoms and the aromatic ring systems.

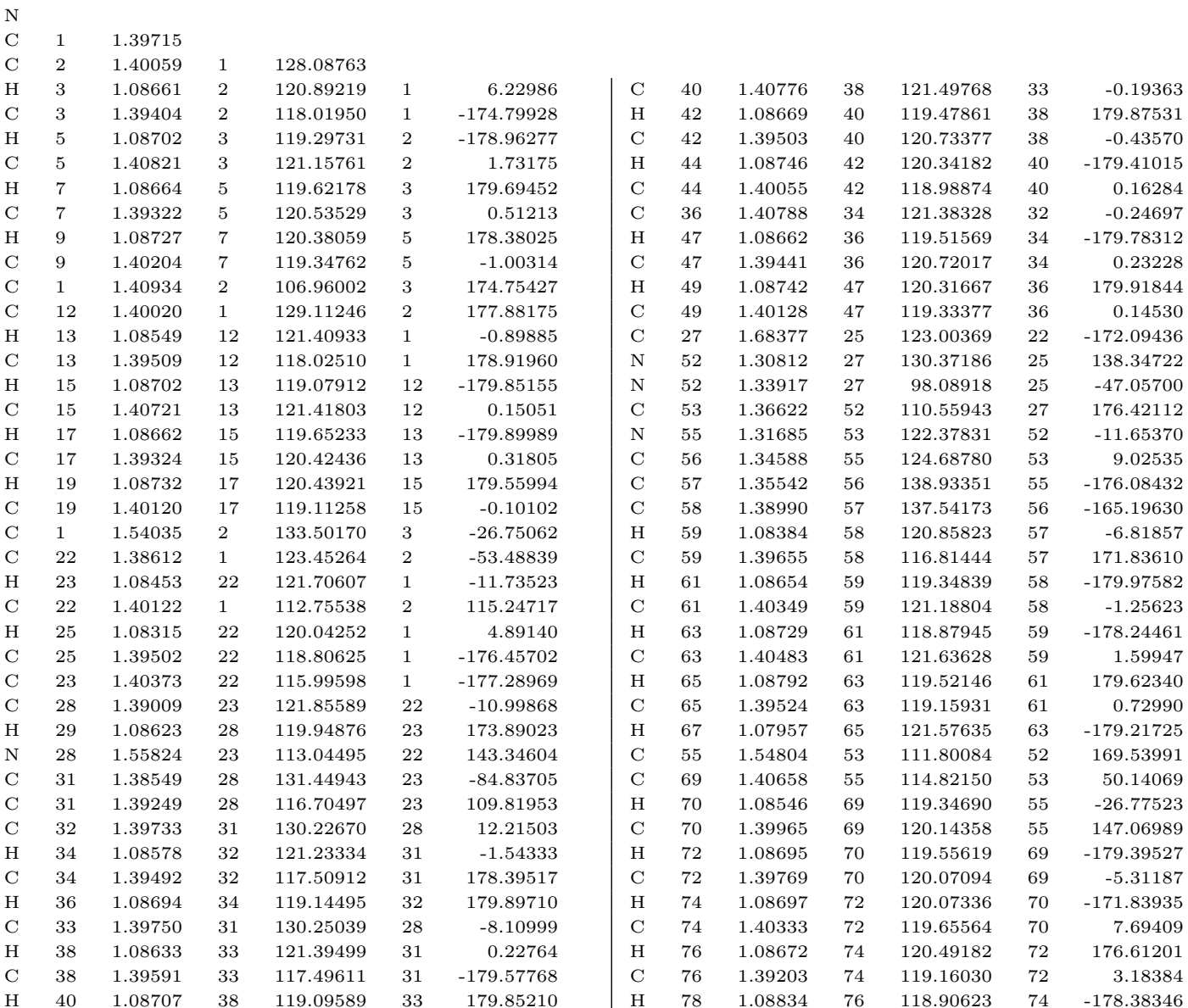


Table S14: Z-matrix of $\mathbf{C z 2 B P}$ at the energy minimum $(\Lambda=\infty)$.

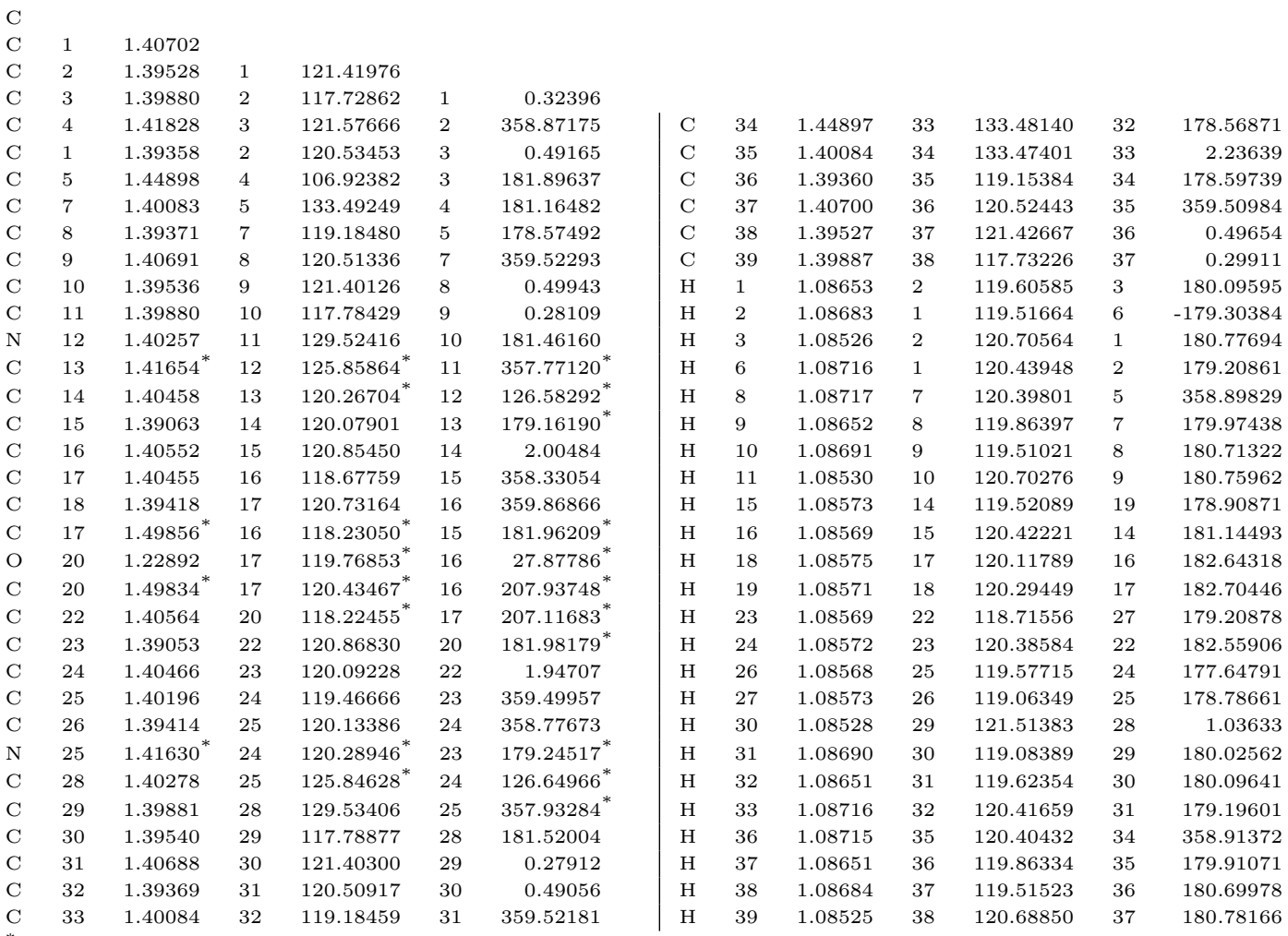


Table S15: Z-matrix of Cz2BP at the TADF maximum with energy penalty $(\Lambda=$

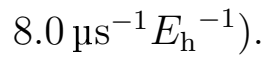

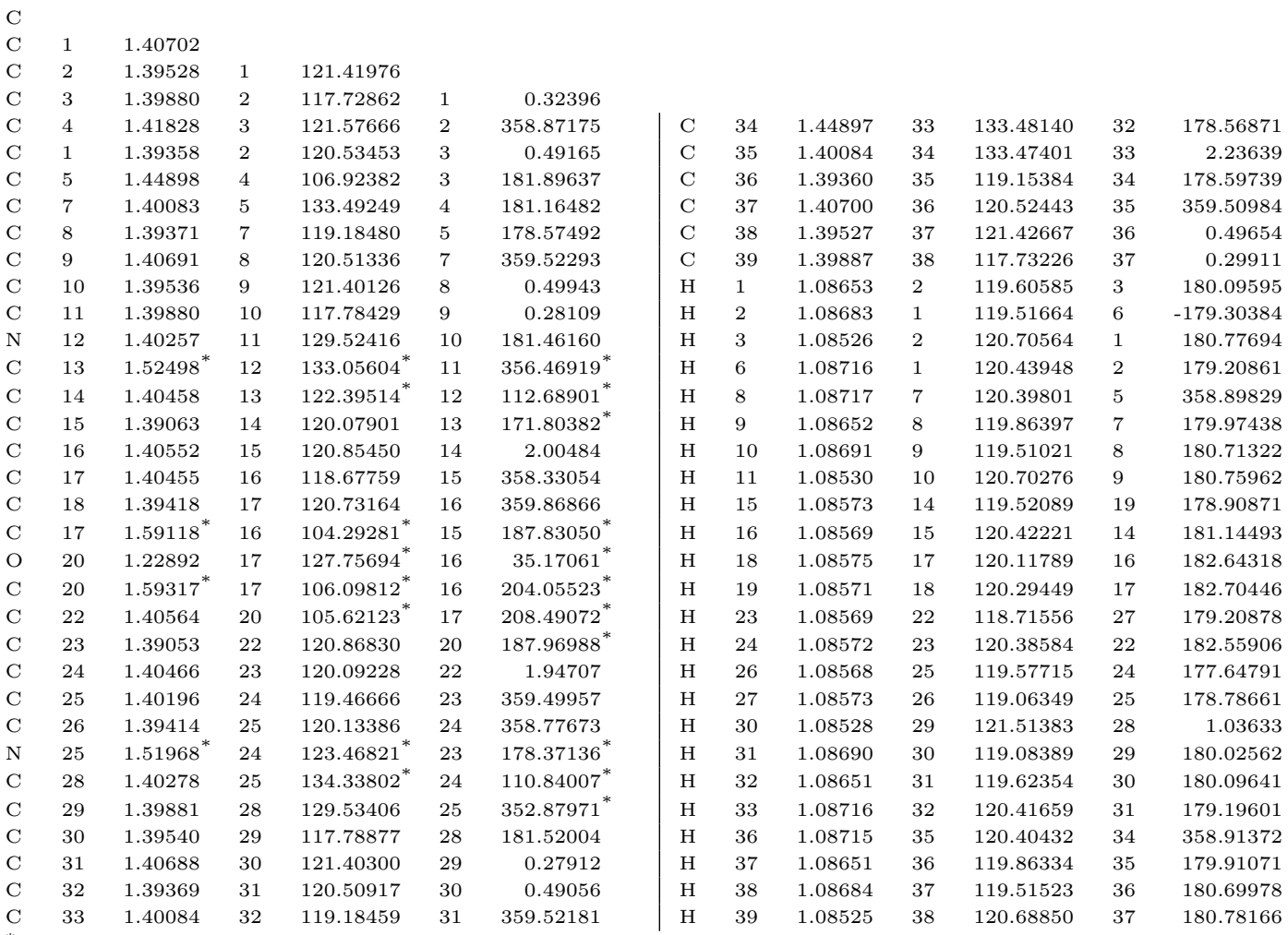


Table S16: Z-matrix of $\mathbf{C z 2 B P}$ at the TADF maximum with energy penalty $(\Lambda=$

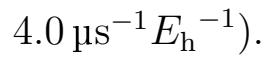

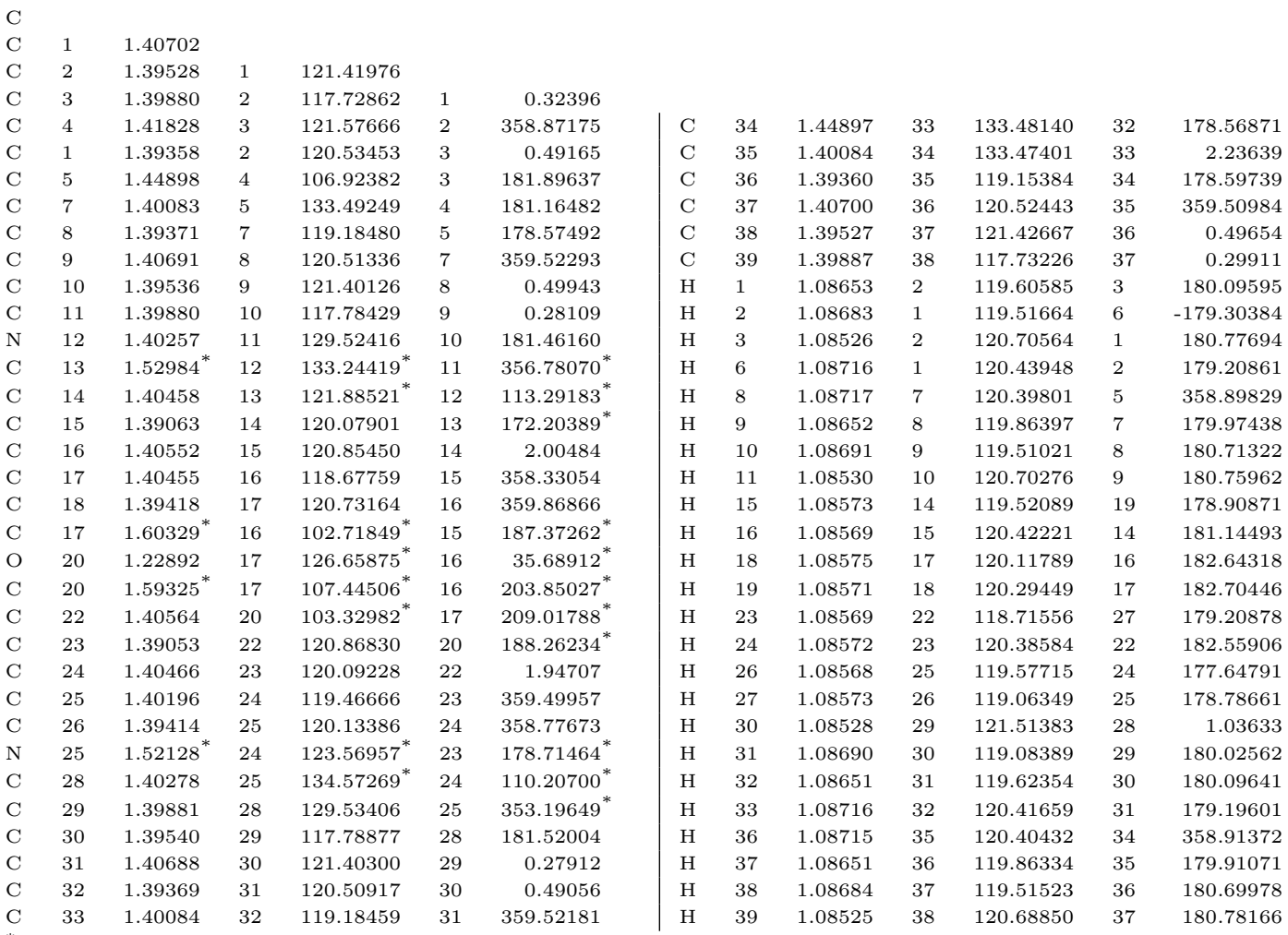


Table S17: Z-matrix of Cz2BP at the TADF maximum with no energy penalty $(\Lambda=0)$.

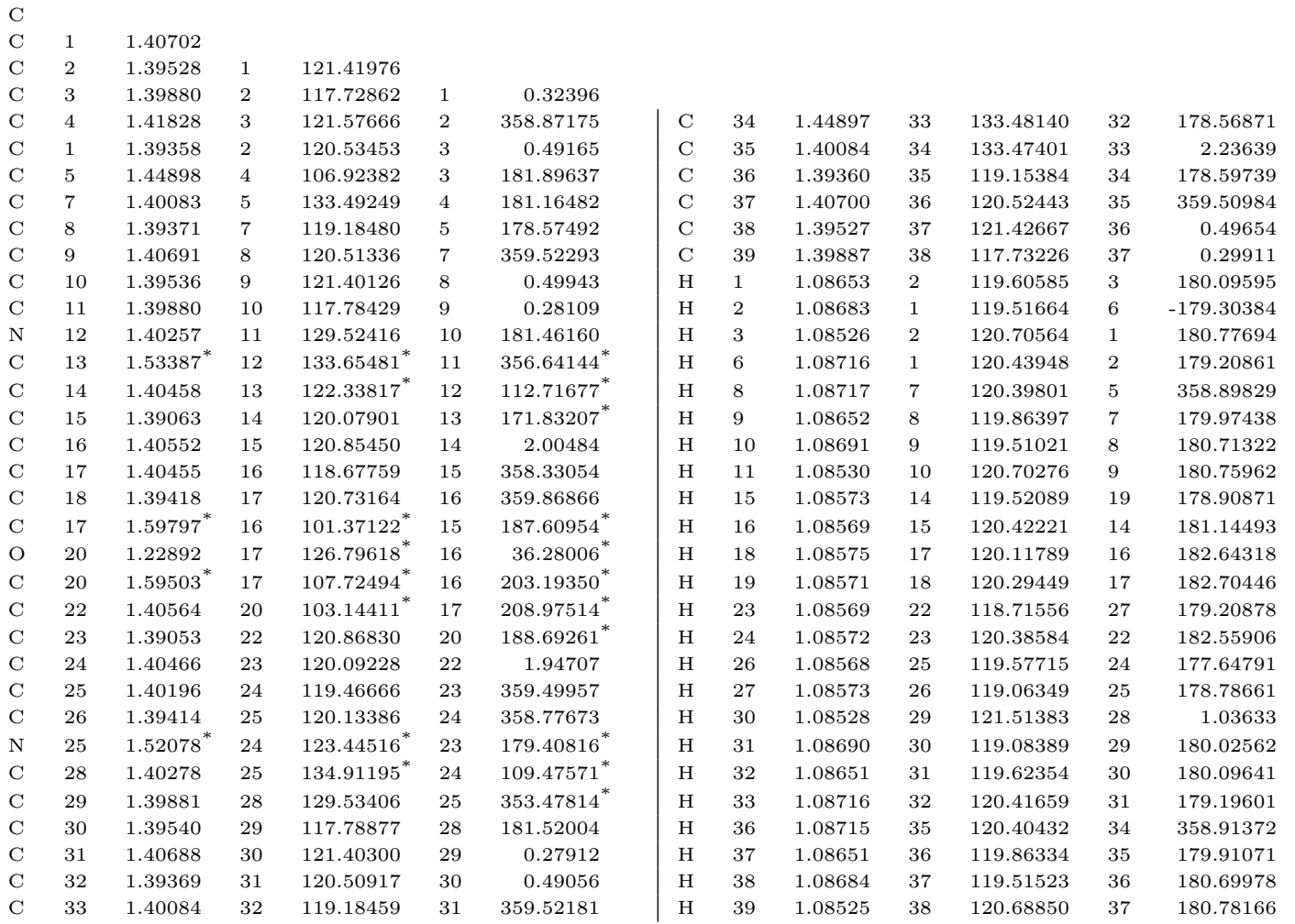

* Z-matrix coordinates that were chosen as variables in the TADF rate maximization. 
Table S18: Z-matrix of $\mathbf{C z 2 B P}$ at the TADF maximum $(\Lambda=0)$ after the relaxation of the hydrogen atoms and the aromatic ring systems.

\begin{tabular}{|c|c|c|c|c|c|c|c|c|c|c|c|c|c|}
\hline \multicolumn{14}{|l|}{$\mathrm{N}$} \\
\hline $\mathrm{C}$ & 1 & 1.39467 & & & & & & & & & & & \\
\hline $\mathrm{C}$ & 2 & 1.40007 & 1 & 128.43849 & & & & & & & & & \\
\hline $\mathrm{H}$ & 3 & 1.08648 & 2 & 121.01022 & 1 & 1.20228 & & & & & & & \\
\hline $\mathrm{C}$ & 3 & 1.39415 & 2 & 117.88388 & 1 & -178.80979 & $\mathrm{C}$ & 34 & 1.38583 & 32 & 134.75808 & 31 & 30.17071 \\
\hline $\mathrm{H}$ & 5 & 1.08697 & 3 & 119.27817 & 2 & -179.81656 & $\mathrm{H}$ & 35 & 1.08578 & 34 & 121.91596 & 32 & -6.70568 \\
\hline $\mathrm{C}$ & 1 & 1.40672 & 2 & 107.33630 & 3 & 177.95123 & $\mathrm{C}$ & 35 & 1.40010 & 34 & 117.11559 & 32 & 169.30457 \\
\hline C & 7 & 1.40020 & 1 & 129.18183 & 2 & 178.99092 & $\mathrm{H}$ & 37 & 1.08564 & 35 & 120.31821 & 34 & -176.42799 \\
\hline $\mathrm{H}$ & 8 & 1.08550 & 7 & 121.48354 & 1 & 0.39734 & $\mathrm{C}$ & 37 & 1.40048 & 35 & 120.50703 & 34 & 0.50967 \\
\hline C & 8 & 1.39504 & 7 & 117.93312 & 1 & -179.57432 & C & 39 & 1.39848 & 37 & 121.62174 & 35 & 2.25902 \\
\hline $\mathrm{H}$ & 10 & 1.08699 & 8 & 119.06799 & 7 & -179.84472 & $\mathrm{H}$ & 40 & 1.08686 & 39 & 119.44551 & 37 & 179.25874 \\
\hline C & 10 & 1.40736 & 8 & 121.43896 & 7 & 0.23061 & C & 40 & 1.39726 & 39 & 118.77815 & 37 & -3.07417 \\
\hline $\mathrm{H}$ & 12 & 1.08660 & 10 & 119.64422 & 8 & -179.96272 & $\mathrm{H}$ & 42 & 1.08182 & 40 & 120.69312 & 39 & -175.83788 \\
\hline $\mathrm{C}$ & 12 & 1.39318 & 10 & 120.45216 & 8 & 0.20760 & $\mathrm{~N}$ & 39 & 1.53387 & 37 & 115.85904 & 35 & -172.95908 \\
\hline $\mathrm{H}$ & 14 & 1.08729 & 12 & 120.45646 & 10 & 179.64437 & $\mathrm{C}$ & 44 & 1.39363 & 39 & 133.65481 & 37 & -72.10270 \\
\hline $\mathrm{C}$ & 14 & 1.40137 & 12 & 119.13279 & 10 & -0.22740 & $\mathrm{C}$ & 45 & 1.39992 & 44 & 128.64143 & 39 & -3.35856 \\
\hline C & 5 & 1.40819 & 3 & 121.19535 & 2 & 0.50517 & $\mathrm{H}$ & 46 & 1.08653 & 45 & 121.05826 & 44 & 0.36681 \\
\hline $\mathrm{H}$ & 17 & 1.08655 & 5 & 119.60927 & 3 & 179.88813 & C & 46 & 1.39428 & 45 & 117.85045 & 44 & -179.45681 \\
\hline $\mathrm{C}$ & 17 & 1.39327 & 5 & 120.56185 & 3 & 0.24361 & $\mathrm{H}$ & 48 & 1.08698 & 46 & 119.26486 & 45 & -179.94844 \\
\hline $\mathrm{H}$ & 19 & 1.08723 & 17 & 120.38633 & 5 & 179.36981 & $\mathrm{C}$ & 44 & 1.40438 & 39 & 118.60592 & 37 & 105.38481 \\
\hline $\mathrm{C}$ & 19 & 1.40207 & 17 & 119.38429 & 5 & -0.35695 & $\mathrm{C}$ & 50 & 1.40011 & 44 & 129.37268 & 39 & 0.76030 \\
\hline $\mathrm{C}$ & 1 & 1.52078 & 2 & 134.91195 & 3 & -6.52186 & $\mathrm{H}$ & 51 & 1.08541 & 50 & 121.49554 & 44 & 0.49755 \\
\hline $\mathrm{C}$ & 22 & 1.39932 & 1 & 123.44516 & 2 & 109.47571 & $\mathrm{C}$ & 51 & 1.39496 & 50 & 117.90476 & 44 & -179.40507 \\
\hline $\mathrm{H}$ & 23 & 1.08718 & 22 & 119.32241 & 1 & -1.44824 & $\mathrm{H}$ & 53 & 1.08699 & 51 & 119.05285 & 50 & -179.89037 \\
\hline $\mathrm{C}$ & 23 & 1.39613 & 22 & 119.28693 & 1 & 179.40816 & $\mathrm{C}$ & 53 & 1.40744 & 51 & 121.45671 & 50 & 0.21337 \\
\hline $\mathrm{H}$ & 25 & 1.08083 & 23 & 120.75467 & 22 & -178.36391 & $\mathrm{H}$ & 55 & 1.08658 & 53 & 119.63983 & 51 & -179.95710 \\
\hline $\mathrm{C}$ & 22 & 1.40154 & 1 & 115.48031 & 2 & -71.26083 & C & 55 & 1.39320 & 53 & 120.45494 & 51 & 0.24104 \\
\hline $\mathrm{H}$ & 27 & 1.08571 & 22 & 119.01719 & 1 & 0.01606 & $\mathrm{H}$ & 57 & 1.08729 & 55 & 120.45551 & 53 & 179.60875 \\
\hline $\mathrm{C}$ & 27 & 1.39929 & 22 & 120.53530 & 1 & -177.54372 & $\mathrm{C}$ & 57 & 1.40143 & 55 & 119.15336 & 53 & -0.24812 \\
\hline $\mathrm{H}$ & 29 & 1.08628 & 27 & 120.66318 & 22 & 175.52679 & $\mathrm{C}$ & 48 & 1.40816 & 46 & 121.21954 & 45 & 0.26876 \\
\hline $\mathrm{C}$ & 29 & 1.38713 & 27 & 117.60677 & 22 & -1.80432 & $\mathrm{H}$ & 60 & 1.08656 & 48 & 119.59809 & 46 & 179.93524 \\
\hline $\mathrm{C}$ & 31 & 1.59503 & 29 & 133.40400 & 27 & 170.83099 & $\mathrm{C}$ & 60 & 1.39344 & 48 & 120.57493 & 46 & 0.14624 \\
\hline $\mathrm{O}$ & 32 & 1.20478 & 31 & 124.09683 & 29 & -155.76847 & $\mathrm{H}$ & 62 & 1.08725 & 60 & 120.38628 & 48 & 179.62986 \\
\hline $\mathrm{C}$ & 32 & 1.59797 & 31 & 107.72495 & 29 & 36.87906 & $\mathrm{C}$ & 62 & 1.40193 & 60 & 119.35952 & 48 & -0.23046 \\
\hline
\end{tabular}


Table S19: Z-matrix of Ac-MPM at the energy minimum $(\Lambda=\infty)$.

\begin{tabular}{|c|c|c|c|c|c|c|c|c|c|c|c|c|c|}
\hline \\
\hline \multicolumn{14}{|c|}{1.50583} \\
\hline $\mathrm{N}$ & 2 & 1.33850 & 1 & 117.65244 & & & & & & & & & \\
\hline $\mathrm{C}$ & 3 & 1.34777 & 2 & 117.53683 & 1 & 180.67270 & $\mathrm{C}$ & 47 & 1.41102 & 46 & 119.50875 & 43 & -179.83838 \\
\hline$C$ & 4 & 1.39892 & 3 & 120.61082 & 2 & 0.41799 & $\mathrm{C}$ & 48 & 1.39122 & 47 & 121.04682 & 46 & -0.04531 \\
\hline C & 5 & 1.40148 & 4 & 118.04691 & 3 & 358.33898 & $\mathrm{C}$ & 49 & 1.39540 & 48 & 120.06786 & 47 & 0.02562 \\
\hline 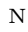 & 6 & 1.34435 & 5 & 120.55549 & 4 & 1.62323 & $\mathrm{C}$ & 50 & 1.39389 & 49 & 118.72214 & 48 & 0.03143 \\
\hline $\mathrm{C}$ & 6 & $1.48802^{*}$ & 5 & $122.56083^{*}$ & 4 & $180.89240^{*}$ & $\mathrm{H}$ & 1 & 1.09183 & 2 & 110.52815 & 3 & 358.89572 \\
\hline $\mathrm{C}$ & 8 & 1.40558 & 6 & $121.90024^{*}$ & 5 & $19.05584^{*}$ & $\mathrm{H}$ & 1 & 1.09596 & 2 & 110.21152 & 3 & 237.75877 \\
\hline C & 9 & 1.39449 & 8 & 120.84388 & 6 & $179.53663^{*}$ & $\mathrm{H}$ & 1 & 1.09643 & 2 & 110.19187 & 3 & 119.89113 \\
\hline 0 & 10 & 1.39844 & 9 & 120.19424 & 8 & 0.05385 & $\mathrm{H}$ & 5 & 1.08254 & 4 & 120.95348 & 3 & 176.04645 \\
\hline C & 11 & 1.39913 & 10 & 119.47151 & 9 & 359.93454 & $\mathrm{H}$ & 9 & 1.08583 & 8 & 120.47543 & 13 & -178.20401 \\
\hline C & 12 & 1.39339 & 11 & 120.28598 & 10 & 359.85119 & $\mathrm{H}$ & 10 & 1.08642 & 9 & 120.48286 & 8 & 180.73154 \\
\hline $\mathrm{N}$ & 11 & $1.43526^{*}$ & 10 & $120.18493^{*}$ & 9 & $180.62019^{*}$ & $\mathrm{H}$ & 12 & 1.08655 & 11 & 119.26092 & 10 & 180.02562 \\
\hline C & 14 & 1.40881 & 11 & $119.03804^{*}$ & 10 & $89.30318^{*}$ & $\mathrm{H}$ & 13 & 1.08450 & 12 & 120.47333 & 11 & 180.62790 \\
\hline C & 15 & 1.41140 & 14 & 120.68266 & 11 & $180.23342^{*}$ & $\mathrm{H}$ & 17 & 1.08648 & 16 & 118.84331 & 15 & 180.02562 \\
\hline C & 16 & 1.40257 & 15 & 117.86250 & 14 & 179.88235 & $\mathrm{H}$ & 18 & 1.08639 & 17 & 120.30937 & 16 & 179.97438 \\
\hline C & 17 & 1.39386 & 16 & 122.78512 & 15 & 0.04971 & $\mathrm{H}$ & 19 & 1.08707 & 18 & 120.64944 & 17 & 179.88956 \\
\hline $\mathrm{C}$ & 18 & 1.39532 & 17 & 118.72603 & 16 & 0.05845 & $\mathrm{H}$ & 20 & 1.08329 & 19 & 119.12749 & 18 & 179.90649 \\
\hline C & 19 & 1.39115 & 18 & 120.05390 & 17 & 359.92503 & $\mathrm{H}$ & 22 & 1.09518 & 21 & 111.14838 & 16 & 301.25129 \\
\hline C & 16 & 1.53400 & 15 & 122.77663 & 14 & 359.87960 & $\mathrm{H}$ & 22 & 1.09524 & 21 & 111.11557 & 16 & 181.42759 \\
\hline C & 21 & 1.55448 & 16 & 109.06563 & 15 & 239.34748 & $\mathrm{H}$ & 22 & 1.09541 & 21 & 110.68218 & 16 & 61.34003 \\
\hline $\mathrm{C}$ & 21 & 1.55432 & 16 & 109.08608 & 15 & 120.20691 & $\mathrm{H}$ & 23 & 1.09534 & 21 & 111.10718 & 16 & 58.89841 \\
\hline C & 21 & 1.53437 & 16 & 111.41054 & 15 & 359.76800 & $\mathrm{H}$ & 23 & 1.09540 & 21 & 110.68742 & 16 & 298.83261 \\
\hline C & 24 & 1.41163 & 21 & 122.76259 & 16 & 0.39126 & $\mathrm{H}$ & 23 & 1.09535 & 21 & 111.12222 & 16 & 178.72351 \\
\hline C & 25 & 1.41099 & 24 & 119.51131 & 21 & 179.83170 & $\mathrm{H}$ & 26 & 1.08348 & 25 & 119.74985 & 24 & -179.99781 \\
\hline $\mathrm{C}$ & 26 & 1.39124 & 25 & 121.06066 & 24 & 0.08325 & $\mathrm{H}$ & 27 & 1.08710 & 26 & 119.30079 & 25 & 179.97438 \\
\hline $\mathrm{C}$ & 27 & 1.39543 & 26 & 120.05288 & 25 & 359.97438 & $\mathrm{H}$ & 28 & 1.08640 & 27 & 120.95898 & 26 & 179.97438 \\
\hline C & 28 & 1.39398 & 27 & 118.72283 & 26 & 359.96278 & $\mathrm{H}$ & 29 & 1.08639 & 28 & 118.41477 & 27 & 180.02562 \\
\hline $\mathrm{C}$ & 4 & $1.48749^{*}$ & 3 & $116.79359^{*}$ & 2 & $179.58587^{*}$ & $\mathrm{H}$ & 31 & 1.08452 & 30 & 118.76935 & 35 & -179.29891 \\
\hline $\mathrm{C}$ & 30 & 1.40577 & 4 & $119.71610^{*}$ & 3 & $341.30782^{*}$ & $\mathrm{H}$ & 32 & 1.08655 & 31 & 120.45969 & 30 & 179.57257 \\
\hline C & 31 & 1.39350 & 30 & 120.76369 & 4 & $180.26535^{*}$ & $\mathrm{H}$ & 34 & 1.08644 & 33 & 119.33823 & 32 & 180.78326 \\
\hline $\mathrm{C}$ & 32 & 1.39900 & 31 & 120.29692 & 30 & 359.53888 & $\mathrm{H}$ & 35 & 1.08590 & 34 & 118.70349 & 33 & 181.45095 \\
\hline $\mathrm{C}$ & 33 & 1.39851 & 32 & 119.47468 & 31 & 359.97355 & $\mathrm{H}$ & 39 & 1.08637 & 38 & 118.79129 & 37 & 180.02562 \\
\hline $\mathrm{C}$ & 34 & 1.39437 & 33 & 120.18402 & 32 & 0.29666 & $\mathrm{H}$ & 40 & 1.08639 & 39 & 120.32407 & 38 & 179.97438 \\
\hline $\mathrm{N}$ & 33 & $1.43519^{*}$ & 32 & $120.31726^{*}$ & 31 & $180.29743^{*}$ & $\mathrm{H}$ & 41 & 1.08710 & 40 & 120.64008 & 39 & 179.97438 \\
\hline $\mathrm{C}$ & 36 & 1.40820 & 33 & $119.15858^{*}$ & 32 & $269.89360^{*}$ & $\mathrm{H}$ & 42 & 1.08344 & 41 & 119.15881 & 40 & 179.97438 \\
\hline $\mathrm{C}$ & 37 & 1.41146 & 36 & 120.69701 & 33 & $180.13193^{*}$ & $\mathrm{H}$ & 44 & 1.09533 & 43 & 111.11629 & 38 & 300.83187 \\
\hline $\mathrm{C}$ & 38 & 1.40242 & 37 & 117.88065 & 36 & 179.97438 & $\mathrm{H}$ & 44 & 1.09539 & 43 & 111.14247 & 38 & 180.96782 \\
\hline $\mathrm{C}$ & 39 & 1.39394 & 38 & 122.78422 & 37 & 0.03726 & $\mathrm{H}$ & 44 & 1.09543 & 43 & 110.67794 & 38 & 60.89850 \\
\hline $\mathrm{C}$ & 40 & 1.39538 & 39 & 118.71530 & 38 & 359.97438 & $\mathrm{H}$ & 45 & 1.09526 & 43 & 111.13974 & 38 & 59.28773 \\
\hline C & 41 & 1.39120 & 40 & 120.06452 & 39 & 359.97438 & $\mathrm{H}$ & 45 & 1.09543 & 43 & 110.68503 & 38 & 299.19004 \\
\hline $\mathrm{C}$ & 38 & 1.53410 & 37 & 122.77223 & 36 & 359.89121 & $\mathrm{H}$ & 45 & 1.09525 & 43 & 111.13650 & 38 & 179.11870 \\
\hline c & 43 & 1.55442 & 38 & 109.04047 & 37 & 239.86052 & $\mathrm{H}$ & 48 & 1.08339 & 47 & 119.80377 & 46 & 179.88065 \\
\hline c & 43 & 1.55435 & 38 & 109.08347 & 37 & 120.72677 & $\mathrm{H}$ & 49 & 1.08707 & 48 & 119.29068 & 47 & 179.97438 \\
\hline & 43 & 1.53418 & 38 & 111.40551 & 37 & 0.26477 & $\mathrm{H}$ & 50 & 1.08640 & 49 & 120.95506 & 48 & 179.97438 \\
\hline & 46 & 1.41147 & 43 & 122.77468 & 38 & -0.28109 & $\mathrm{H}$ & 51 & 1.08643 & 50 & 118.43815 & 49 & 179.97438 \\
\hline
\end{tabular}


Table S20: Z-matrix of Ac-MPM at the TADF maximum with energy penalty $(\Lambda=$ $\left.8.0 \mathrm{\mu s}^{-1} E_{\mathrm{h}}{ }^{-1}\right)$.

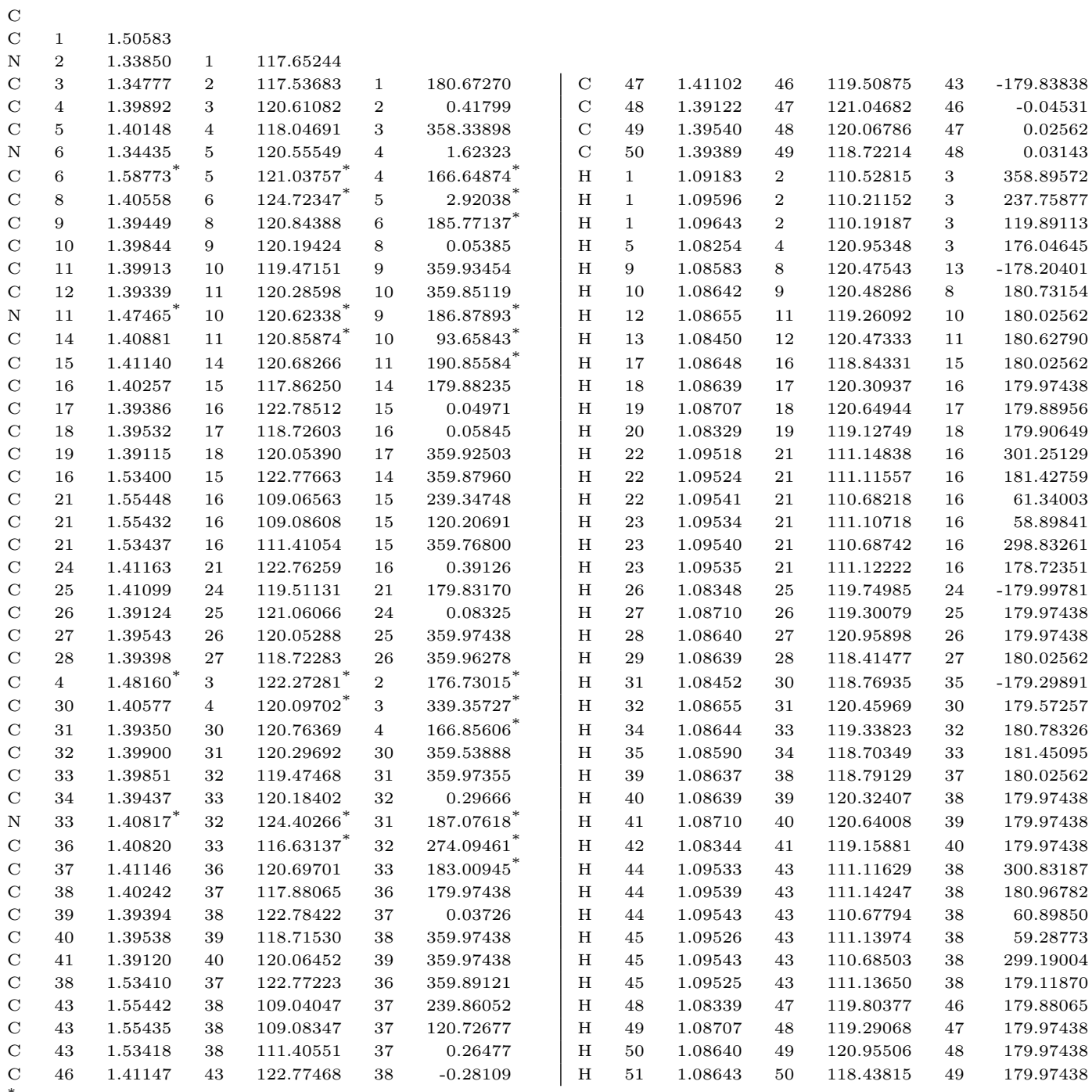


Table S21: Z-matrix of Ac-MPM at the TADF maximum with energy penalty $(\Lambda=$ $\left.4.0 \mathrm{\mu s}^{-1} E_{\mathrm{h}}{ }^{-1}\right)$.

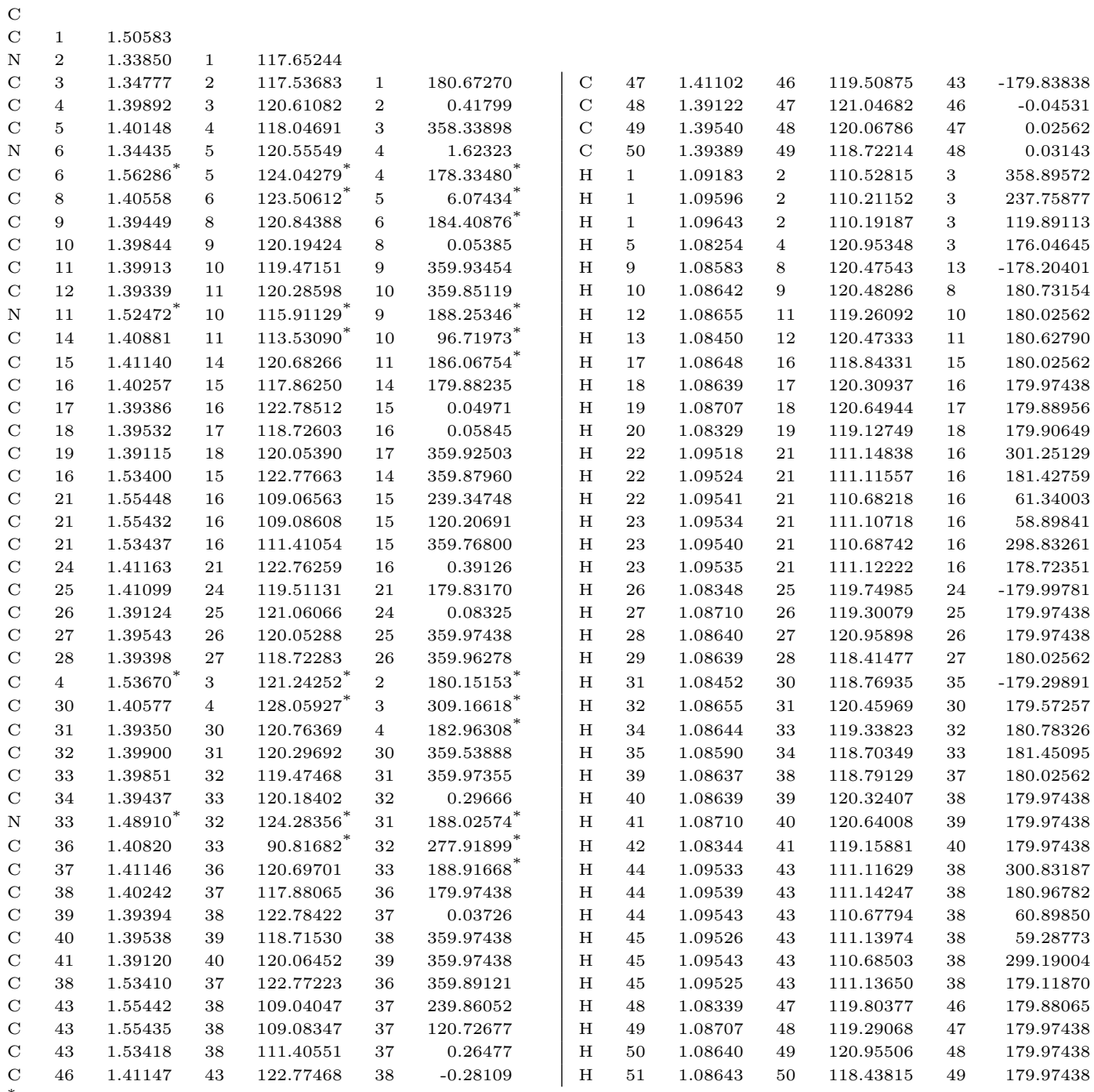


Table S22: Z-matrix of Ac-MPM at the TADF maximum with no energy penalty $(\Lambda=0)$.

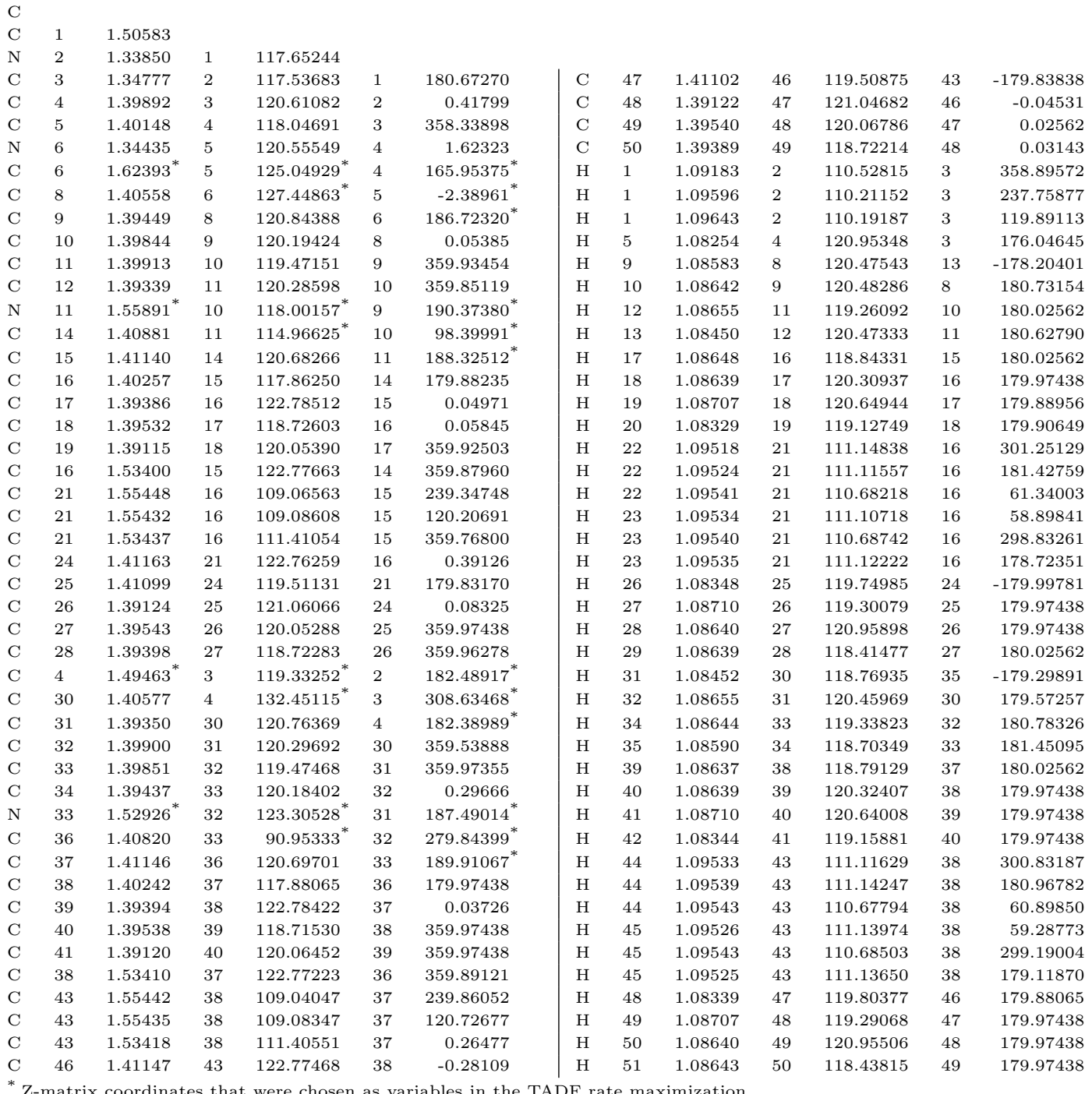


Table S23: Z-matrix of Ac-MPM at the TADF maximum $(\Lambda=0)$ after the relaxation of the hydrogen atoms and the aromatic ring systems.

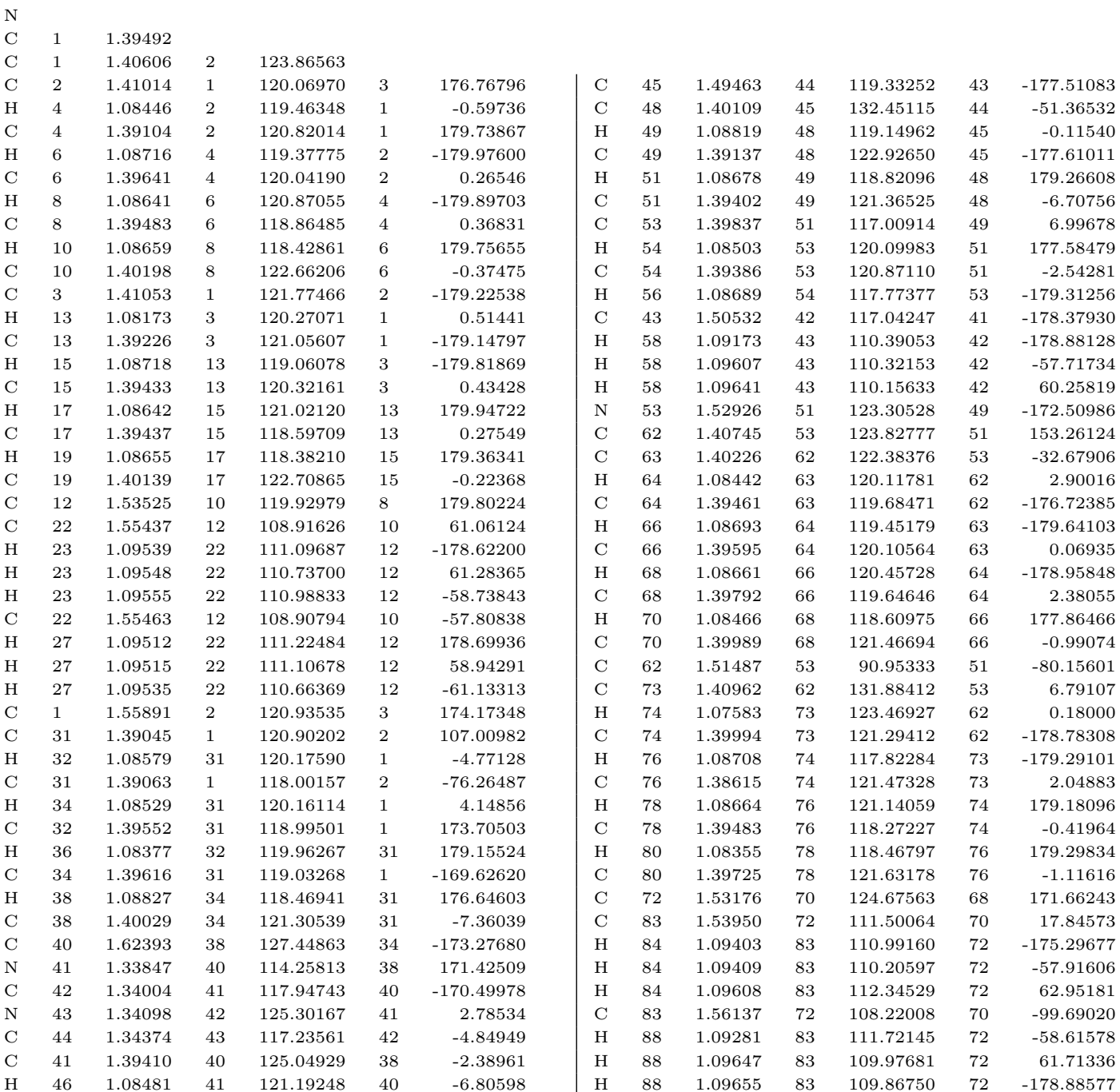


Table S24: Z-matrix of SpiroAC-TRZ at the energy minimum $(\Lambda=\infty)$.

\begin{tabular}{|c|c|c|c|c|c|c|c|c|c|c|c|c|c|}
\hline$\tau$ & & & & & & & & & & & & & \\
\hline $\mathrm{C}$ & 1 & 1.39864 & & & & & & & & & & & \\
\hline $\mathrm{C}$ & 2 & 1.39443 & 1 & 120.16574 & & & & & & & & & \\
\hline $\mathrm{C}$ & 3 & 1.40511 & 2 & 120.36404 & 1 & 0.02562 & & & & & & & \\
\hline $\mathrm{C}$ & 4 & 1.40517 & 3 & 119.15050 & 2 & 359.97438 & $\mathrm{C}$ & 42 & 1.40158 & 41 & 120.58139 & 40 & 359.97438 \\
\hline $\mathrm{C}$ & 5 & 1.39446 & 4 & 120.35081 & 3 & 359.97438 & $\mathrm{C}$ & 43 & 1.40051 & 42 & 120.50910 & 41 & -0.01063 \\
\hline C & 4 & $1.48397^{*}$ & 3 & $120.41209^{*}$ & 2 & $180.02562^{*}$ & $\mathrm{C}$ & 40 & $1.47063^{*}$ & 39 & $108.64466^{*}$ & 44 & $179.96928^{*}$ \\
\hline $\mathrm{N}$ & 7 & 1.34295 & 4 & $117.98695^{*}$ & 3 & $0.24845^{*}$ & $\mathrm{C}$ & 45 & 1.40777 & 40 & $108.62415^{*}$ & 39 & $0.02562^{*}$ \\
\hline $\mathrm{C}$ & 8 & 1.34095 & 7 & 115.86057 & 4 & $179.86002^{*}$ & $\mathrm{C}$ & 46 & 1.39054 & 45 & 120.59798 & 40 & $180.02562^{*}$ \\
\hline $\mathrm{N}$ & 9 & 1.34093 & 8 & 124.28585 & 7 & 0.15796 & $\mathrm{C}$ & 47 & 1.40062 & 46 & 119.00287 & 45 & 359.97438 \\
\hline $\mathrm{C}$ & 10 & 1.34317 & 9 & 115.83276 & 8 & 359.89831 & $\mathrm{C}$ & 48 & 1.40125 & 47 & 120.52755 & 46 & 359.97438 \\
\hline $\mathrm{N}$ & 11 & 1.34192 & 10 & 123.98178 & 9 & -0.04540 & $\mathrm{C}$ & 49 & 1.39825 & 48 & 120.55950 & 47 & 0.03294 \\
\hline $\mathrm{C}$ & 11 & $1.48397^{*}$ & 10 & $118.00583^{*}$ & 9 & $180.02562^{*}$ & $\mathrm{H}$ & 1 & 1.08713 & 2 & 120.10348 & 3 & 179.97438 \\
\hline $\mathrm{C}$ & 13 & 1.40515 & 11 & $120.45847^{*}$ & 10 & $359.59226^{*}$ & $\mathrm{H}$ & 2 & 1.08693 & 1 & 120.09391 & 3 & 180.02562 \\
\hline C & 14 & 1.39448 & 13 & 120.35439 & 11 & $179.97438^{*}$ & $\mathrm{H}$ & 3 & 1.08427 & 2 & 120.59577 & 1 & 180.02562 \\
\hline C & 15 & 1.39867 & 14 & 120.16896 & 13 & 359.97438 & $\mathrm{H}$ & 5 & 1.08430 & 4 & 119.02626 & 3 & 179.97438 \\
\hline $\mathrm{C}$ & 16 & 1.39868 & 15 & 119.79481 & 14 & 0.02562 & $\mathrm{H}$ & 6 & 1.08694 & 5 & 119.73537 & 4 & 180.02562 \\
\hline C & 17 & 1.39438 & 16 & 120.17167 & 15 & 0.02562 & $\mathrm{H}$ & 14 & 1.08428 & 13 & 119.03859 & 18 & 179.95982 \\
\hline $\mathrm{C}$ & 9 & $1.48607^{*}$ & 8 & $117.80484^{*}$ & 7 & $180.32489^{*}$ & $\mathrm{H}$ & 15 & 1.08693 & 14 & 119.73768 & 13 & 179.97438 \\
\hline C & 19 & 1.40442 & 9 & $120.43777^{*}$ & 8 & $359.63072^{*}$ & $\mathrm{H}$ & 16 & 1.08713 & 15 & 120.09936 & 14 & 180.02562 \\
\hline $\mathrm{C}$ & 20 & 1.39361 & 19 & 120.44951 & 9 & $179.64292^{*}$ & $\mathrm{H}$ & 17 & 1.08694 & 16 & 120.08859 & 15 & 180.02562 \\
\hline C & 21 & 1.39882 & 20 & 120.21264 & 19 & 359.97438 & $\mathrm{H}$ & 18 & 1.08428 & 17 & 120.62263 & 16 & 179.97438 \\
\hline C & 22 & 1.39970 & 21 & 119.67264 & 20 & 0.15218 & $\mathrm{H}$ & 20 & 1.08418 & 19 & 119.09929 & 24 & -179.99032 \\
\hline $\mathrm{C}$ & 23 & 1.39353 & 22 & 120.16856 & 21 & 359.85828 & $\mathrm{H}$ & 21 & 1.08639 & 20 & 120.54881 & 19 & 180.13849 \\
\hline $\mathrm{N}$ & 22 & $1.43589^{*}$ & 21 & $119.88295^{*}$ & 20 & $180.45092^{*}$ & $\mathrm{H}$ & 23 & 1.08648 & 22 & 119.31321 & 21 & 179.97438 \\
\hline $\mathrm{C}$ & 25 & $1.40845^{*}$ & 22 & $119.15861^{*}$ & 21 & $91.66166^{*}$ & $\mathrm{H}$ & 24 & 1.08427 & 23 & 120.43211 & 22 & 180.12133 \\
\hline $\mathrm{C}$ & 26 & 1.40940 & 25 & $120.78333^{*}$ & 22 & $181.82435^{*}$ & $\mathrm{H}$ & 28 & 1.08664 & 27 & 118.40112 & 26 & 180.02562 \\
\hline $\mathrm{C}$ & 27 & 1.40190 & 26 & 118.64931 & 25 & $180.26182^{*}$ & $\mathrm{H}$ & 29 & 1.08626 & 28 & 120.33044 & 27 & 180.14617 \\
\hline C & 28 & 1.39189 & 27 & 122.26296 & 26 & 359.76559 & $\mathrm{H}$ & 30 & 1.08710 & 29 & 120.53882 & 28 & 180.02562 \\
\hline $\mathrm{C}$ & 29 & 1.39740 & 28 & 118.76420 & 27 & 359.97438 & $\mathrm{H}$ & 31 & 1.08354 & 30 & 119.21243 & 29 & 179.76689 \\
\hline $\mathrm{C}$ & 30 & 1.39055 & 29 & 120.26866 & 28 & 0.20004 & $\mathrm{H}$ & 34 & 1.08358 & 33 & 119.76961 & 38 & -179.94501 \\
\hline $\mathrm{C}$ & 27 & $1.53331^{*}$ & 26 & $122.20037^{*}$ & 25 & $2.53202^{*}$ & $\mathrm{H}$ & 35 & 1.08709 & 34 & 119.20891 & 33 & 179.97438 \\
\hline $\mathrm{C}$ & 25 & $1.40831^{*}$ & 22 & $119.29754^{*}$ & 21 & $267.79859^{*}$ & $\mathrm{H}$ & 36 & 1.08626 & 35 & 120.89328 & 34 & 179.97438 \\
\hline $\mathrm{C}$ & 33 & 1.41195 & 25 & $120.09902^{*}$ & 22 & $358.19701^{*}$ & $\mathrm{H}$ & 37 & 1.08669 & 36 & 119.32083 & 35 & 180.33624 \\
\hline C & 34 & 1.39060 & 33 & 120.94302 & 25 & $180.13352^{*}$ & $\mathrm{H}$ & 41 & 1.08750 & 40 & 120.85913 & 39 & 180.02562 \\
\hline $\mathrm{C}$ & 35 & 1.39745 & 34 & 120.25391 & 33 & 0.10523 & $\mathrm{H}$ & 42 & 1.08715 & 41 & 119.71334 & 40 & 179.97438 \\
\hline $\mathrm{C}$ & 36 & 1.39186 & 35 & 118.78061 & 34 & 359.78995 & $\mathrm{H}$ & 43 & 1.08705 & 42 & 119.74014 & 41 & 179.97438 \\
\hline $\mathrm{C}$ & 37 & 1.40196 & 36 & 122.26388 & 35 & 0.05250 & $\mathrm{H}$ & 44 & 1.08743 & 43 & 120.37776 & 42 & -179.99848 \\
\hline C & 32 & $1.53844^{*}$ & 27 & $111.35488^{*}$ & 26 & $225.32217^{*}$ & $\mathrm{H}$ & 47 & 1.08744 & 46 & 120.72099 & 45 & 179.97438 \\
\hline $\mathrm{C}$ & 39 & 1.40745 & 32 & $111.13284^{*}$ & 27 & $242.74082^{*}$ & $\mathrm{H}$ & 48 & 1.08708 & 47 & 119.73759 & 46 & 180.02562 \\
\hline $\mathrm{C}$ & 40 & 1.39861 & 39 & 120.41622 & 32 & $179.97438^{*}$ & $\mathrm{H}$ & 49 & 1.08711 & 48 & 119.71986 & 47 & 180.02562 \\
\hline $\mathrm{C}$ & 41 & 1.39810 & 40 & 118.87226 & 39 & 0.02562 & $\mathrm{H}$ & 50 & 1.08755 & 49 & 120.25204 & 48 & 179.97980 \\
\hline
\end{tabular}


Table S25: Z-matrix of SpiroAC-TRZ at the TADF maximum with energy penalty $(\Lambda=$

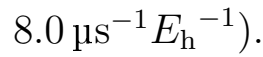

\begin{tabular}{|c|c|c|c|c|c|c|c|c|c|c|c|c|c|}
\hline C & 1 & 1.39864 & & & & & & & & & & & \\
\hline $\mathrm{C}$ & 2 & 1.39443 & 1 & 120.16574 & & & & & & & & & \\
\hline $\mathrm{C}$ & 3 & 1.40511 & 2 & 120.36404 & 1 & 0.02562 & & & & & & & \\
\hline $\mathrm{C}$ & 4 & 1.40517 & 3 & 119.15050 & 2 & 359.97438 & $\mathrm{C}$ & 42 & 1.40158 & 41 & 120.58139 & 40 & 359.97438 \\
\hline $\mathrm{C}$ & 5 & 1.39446 & 4 & 120.35081 & 3 & 359.97438 & $\mathrm{C}$ & 43 & 1.40051 & 42 & 120.50910 & 41 & -0.01063 \\
\hline $\mathrm{C}$ & 4 & $1.49978^{*}$ & 3 & $124.94879^{*}$ & 2 & $173.42101^{*}$ & $\mathrm{C}$ & 40 & $1.48760^{*}$ & 39 & $107.69736^{*}$ & 44 & $189.99573^{*}$ \\
\hline $\mathrm{N}$ & 7 & 1.34295 & 4 & $108.77141^{*}$ & 3 & $0.27177^{*}$ & $\mathrm{C}$ & 45 & 1.40777 & 40 & $107.38631^{*}$ & 39 & $0.75640^{*}$ \\
\hline $\mathrm{C}$ & 8 & 1.34095 & 7 & 115.86057 & 4 & $180.26046^{*}$ & $\mathrm{C}$ & 46 & 1.39054 & 45 & 120.59798 & 40 & $180.78082^{*}$ \\
\hline $\mathrm{N}$ & 9 & 1.34093 & 8 & 124.28585 & 7 & 0.15796 & C & 47 & 1.40062 & 46 & 119.00287 & 45 & 359.97438 \\
\hline $\mathrm{C}$ & 10 & 1.34317 & 9 & 115.83276 & 8 & 359.89831 & $\mathrm{C}$ & 48 & 1.40125 & 47 & 120.52755 & 46 & 359.97438 \\
\hline $\mathrm{N}$ & 11 & 1.34192 & 10 & 123.98178 & 9 & -0.04540 & $\mathrm{C}$ & 49 & 1.39825 & 48 & 120.55950 & 47 & 0.03294 \\
\hline $\mathrm{C}$ & 11 & $1.58946^{*}$ & 10 & $118.14211^{*}$ & 9 & $179.30103^{*}$ & $\mathrm{H}$ & 1 & 1.08713 & 2 & 120.10348 & 3 & 179.97438 \\
\hline $\mathrm{C}$ & 13 & 1.40515 & 11 & $120.84298^{*}$ & 10 & $372.87065^{*}$ & $\mathrm{H}$ & 2 & 1.08693 & 1 & 120.09391 & 3 & 180.02562 \\
\hline $\mathrm{C}$ & 14 & 1.39448 & 13 & 120.35439 & 11 & $167.44939^{*}$ & $\mathrm{H}$ & 3 & 1.08427 & 2 & 120.59577 & 1 & 180.02562 \\
\hline $\mathrm{C}$ & 15 & 1.39867 & 14 & 120.16896 & 13 & 359.97438 & $\mathrm{H}$ & 5 & 1.08430 & 4 & 119.02626 & 3 & 179.97438 \\
\hline C & 16 & 1.39868 & 15 & 119.79481 & 14 & 0.02562 & $\mathrm{H}$ & 6 & 1.08694 & 5 & 119.73537 & 4 & 180.02562 \\
\hline $\mathrm{C}$ & 17 & 1.39438 & 16 & 120.17167 & 15 & 0.02562 & $\mathrm{H}$ & 14 & 1.08428 & 13 & 119.03859 & 18 & 179.95982 \\
\hline $\mathrm{C}$ & 9 & $1.58104^{*}$ & 8 & $122.52945^{*}$ & 7 & $182.48646^{*}$ & $\mathrm{H}$ & 15 & 1.08693 & 14 & 119.73768 & 13 & 179.97438 \\
\hline $\mathrm{C}$ & 19 & 1.40442 & 9 & $116.79138^{*}$ & 8 & $361.16568^{*}$ & $\mathrm{H}$ & 16 & 1.08713 & 15 & 120.09936 & 14 & 180.02562 \\
\hline $\mathrm{C}$ & 20 & 1.39361 & 19 & 120.44951 & 9 & $176.57457^{*}$ & $\mathrm{H}$ & 17 & 1.08694 & 16 & 120.08859 & 15 & 180.02562 \\
\hline $\mathrm{C}$ & 21 & 1.39882 & 20 & 120.21264 & 19 & 359.97438 & $\mathrm{H}$ & 18 & 1.08428 & 17 & 120.62263 & 16 & 179.97438 \\
\hline $\mathrm{C}$ & 22 & 1.39970 & 21 & 119.67264 & 20 & 0.15218 & $\mathrm{H}$ & 20 & 1.08418 & 19 & 119.09929 & 24 & -179.99032 \\
\hline $\mathrm{C}$ & 23 & 1.39353 & 22 & 120.16856 & 21 & 359.85828 & $\mathrm{H}$ & 21 & 1.08639 & 20 & 120.54881 & 19 & 180.13849 \\
\hline $\mathrm{N}$ & 22 & $1.39728^{*}$ & 21 & $121.04382^{*}$ & 20 & $185.09586^{*}$ & $\mathrm{H}$ & 23 & 1.08648 & 22 & 119.31321 & 21 & 179.97438 \\
\hline $\mathrm{C}$ & 25 & $1.42786^{*}$ & 22 & $114.89611^{*}$ & 21 & $94.81547^{*}$ & $\mathrm{H}$ & 24 & 1.08427 & 23 & 120.43211 & 22 & 180.12133 \\
\hline $\mathrm{C}$ & 26 & 1.40940 & 25 & $119.71894^{*}$ & 22 & $181.45209^{*}$ & $\mathrm{H}$ & 28 & 1.08664 & 27 & 118.40112 & 26 & 180.02562 \\
\hline $\mathrm{C}$ & 27 & 1.40190 & 26 & 118.64931 & 25 & $179.68903^{*}$ & $\mathrm{H}$ & 29 & 1.08626 & 28 & 120.33044 & 27 & 180.14617 \\
\hline C & 28 & 1.39189 & 27 & 122.26296 & 26 & 359.76559 & $\mathrm{H}$ & 30 & 1.08710 & 29 & 120.53882 & 28 & 180.02562 \\
\hline $\mathrm{C}$ & 29 & 1.39740 & 28 & 118.76420 & 27 & 359.97438 & $\mathrm{H}$ & 31 & 1.08354 & 30 & 119.21243 & 29 & 179.76689 \\
\hline C & 30 & 1.39055 & 29 & 120.26866 & 28 & 0.20004 & $\mathrm{H}$ & 34 & 1.08358 & 33 & 119.76961 & 38 & -179.94501 \\
\hline $\mathrm{C}$ & 27 & $1.53922^{*}$ & 26 & $119.94814^{*}$ & 25 & $5.69563^{*}$ & $\mathrm{H}$ & 35 & 1.08709 & 34 & 119.20891 & 33 & 179.97438 \\
\hline $\mathrm{C}$ & 25 & $1.43477^{*}$ & 22 & $121.02129^{*}$ & 21 & $275.12326^{*}$ & $\mathrm{H}$ & 36 & 1.08626 & 35 & 120.89328 & 34 & 179.97438 \\
\hline $\mathrm{C}$ & 33 & 1.41195 & 25 & $122.53828^{*}$ & 22 & $360.56800^{*}$ & $\mathrm{H}$ & 37 & 1.08669 & 36 & 119.32083 & 35 & 180.33624 \\
\hline $\mathrm{C}$ & 34 & 1.39060 & 33 & 120.94302 & 25 & $178.78098^{*}$ & $\mathrm{H}$ & 41 & 1.08750 & 40 & 120.85913 & 39 & 180.02562 \\
\hline $\mathrm{C}$ & 35 & 1.39745 & 34 & 120.25391 & 33 & 0.10523 & $\mathrm{H}$ & 42 & 1.08715 & 41 & 119.71334 & 40 & 179.97438 \\
\hline $\mathrm{C}$ & 36 & 1.39186 & 35 & 118.78061 & 34 & 359.78995 & $\mathrm{H}$ & 43 & 1.08705 & 42 & 119.74014 & 41 & 179.97438 \\
\hline $\mathrm{C}$ & 37 & 1.40196 & 36 & 122.26388 & 35 & 0.05250 & $\mathrm{H}$ & 44 & 1.08743 & 43 & 120.37776 & 42 & -179.99848 \\
\hline $\mathrm{C}$ & 32 & $1.51266^{*}$ & 27 & $107.26540^{*}$ & 26 & $222.26042^{*}$ & $\mathrm{H}$ & 47 & 1.08744 & 46 & 120.72099 & 45 & 179.97438 \\
\hline $\mathrm{C}$ & 39 & 1.40745 & 32 & $110.92204^{*}$ & 27 & $227.29839^{*}$ & $\mathrm{H}$ & 48 & 1.08708 & 47 & 119.73759 & 46 & 180.02562 \\
\hline $\mathrm{C}$ & 40 & 1.39861 & 39 & 120.41622 & 32 & $175.77397^{*}$ & $\mathrm{H}$ & 49 & 1.08711 & 48 & 119.71986 & 47 & 180.02562 \\
\hline $\mathrm{C}$ & 41 & 1.39810 & 40 & 118.87226 & 39 & 0.02562 & $\mathrm{H}$ & 50 & 1.08755 & 49 & 120.25204 & 48 & 179.97980 \\
\hline
\end{tabular}


Table S26: Z-matrix of SpiroAC-TRZ at the TADF maximum with energy penalty $(\Lambda=$ $\left.4.0 \mathrm{\mu s}^{-1} E_{\mathrm{h}}{ }^{-1}\right)$.

\begin{tabular}{|c|c|c|c|c|c|c|c|c|c|c|c|c|c|}
\hline $\mathrm{C}$ & 1 & 1.39864 & & & & & & & & & & & \\
\hline $\mathrm{C}$ & 2 & 1.39443 & 1 & 120.16574 & & & & & & & & & \\
\hline $\mathrm{C}$ & 3 & 1.40511 & 2 & 120.36404 & 1 & 0.02562 & & & & & & & \\
\hline C & 4 & 1.40517 & 3 & 119.15050 & 2 & 359.97438 & $\mathrm{C}$ & 42 & 1.40158 & 41 & 120.58139 & 40 & 359.97438 \\
\hline $\mathrm{C}$ & 5 & 1.39446 & 4 & 120.35081 & 3 & 359.97438 & $\mathrm{C}$ & 43 & 1.40051 & 42 & 120.50910 & 41 & -0.01063 \\
\hline C & 4 & $1.46986^{*}$ & 3 & $118.86981^{*}$ & 2 & $181.49791^{*}$ & $\mathrm{C}$ & 40 & $1.44129^{*}$ & 39 & $109.00697^{*}$ & 44 & $176.22851^{*}$ \\
\hline $\mathrm{N}$ & 7 & 1.34295 & 4 & $112.02339^{*}$ & 3 & $1.54776^{*}$ & $\mathrm{C}$ & 45 & 1.40777 & 40 & $106.13967^{*}$ & 39 & $0.22415^{*}$ \\
\hline $\mathrm{C}$ & 8 & 1.34095 & 7 & 115.86057 & 4 & $180.05888^{*}$ & $\mathrm{C}$ & 46 & 1.39054 & 45 & 120.59798 & 40 & $181.07432^{*}$ \\
\hline $\mathrm{N}$ & 9 & 1.34093 & 8 & 124.28585 & 7 & 0.15796 & $\mathrm{C}$ & 47 & 1.40062 & 46 & 119.00287 & 45 & 359.97438 \\
\hline $\mathrm{C}$ & 10 & 1.34317 & 9 & 115.83276 & 8 & 359.89831 & $\mathrm{C}$ & 48 & 1.40125 & 47 & 120.52755 & 46 & 359.97438 \\
\hline $\mathrm{N}$ & 11 & 1.34192 & 10 & 123.98178 & 9 & -0.04540 & $\mathrm{C}$ & 49 & 1.39825 & 48 & 120.55950 & 47 & 0.03294 \\
\hline C & 11 & $1.66172^{*}$ & 10 & $119.62692^{*}$ & 9 & $180.44673^{*}$ & $\mathrm{H}$ & 1 & 1.08713 & 2 & 120.10348 & 3 & 179.97438 \\
\hline $\mathrm{C}$ & 13 & 1.40515 & 11 & $123.23214^{*}$ & 10 & $359.40282^{*}$ & $\mathrm{H}$ & 2 & 1.08693 & 1 & 120.09391 & 3 & 180.02562 \\
\hline $\mathrm{C}$ & 14 & 1.39448 & 13 & 120.35439 & 11 & $179.64214^{*}$ & $\mathrm{H}$ & 3 & 1.08427 & 2 & 120.59577 & 1 & 180.02562 \\
\hline $\mathrm{C}$ & 15 & 1.39867 & 14 & 120.16896 & 13 & 359.97438 & $\mathrm{H}$ & 5 & 1.08430 & 4 & 119.02626 & 3 & 179.97438 \\
\hline $\mathrm{C}$ & 16 & 1.39868 & 15 & 119.79481 & 14 & 0.02562 & $\mathrm{H}$ & 6 & 1.08694 & 5 & 119.73537 & 4 & 180.02562 \\
\hline $\mathrm{C}$ & 17 & 1.39438 & 16 & 120.17167 & 15 & 0.02562 & $\mathrm{H}$ & 14 & 1.08428 & 13 & 119.03859 & 18 & 179.95982 \\
\hline $\mathrm{C}$ & 9 & $1.51262^{*}$ & 8 & $116.29851^{*}$ & 7 & $181.09967^{*}$ & $\mathrm{H}$ & 15 & 1.08693 & 14 & 119.73768 & 13 & 179.97438 \\
\hline C & 19 & 1.40442 & 9 & $118.25573^{*}$ & 8 & $360.39622^{*}$ & $\mathrm{H}$ & 16 & 1.08713 & 15 & 120.09936 & 14 & 180.02562 \\
\hline $\mathrm{C}$ & 20 & 1.39361 & 19 & 120.44951 & 9 & $180.22357^{*}$ & $\mathrm{H}$ & 17 & 1.08694 & 16 & 120.08859 & 15 & 180.02562 \\
\hline C & 21 & 1.39882 & 20 & 120.21264 & 19 & 359.97438 & $\mathrm{H}$ & 18 & 1.08428 & 17 & 120.62263 & 16 & 179.97438 \\
\hline $\mathrm{C}$ & 22 & 1.39970 & 21 & 119.67264 & 20 & 0.15218 & $\mathrm{H}$ & 20 & 1.08418 & 19 & 119.09929 & 24 & -179.99032 \\
\hline $\mathrm{C}$ & 23 & 1.39353 & 22 & 120.16856 & 21 & 359.85828 & $\mathrm{H}$ & 21 & 1.08639 & 20 & 120.54881 & 19 & 180.13849 \\
\hline $\mathrm{N}$ & 22 & $1.33022^{*}$ & 21 & $117.27093^{*}$ & 20 & $185.06677^{*}$ & $\mathrm{H}$ & 23 & 1.08648 & 22 & 119.31321 & 21 & 179.97438 \\
\hline $\mathrm{C}$ & 25 & $1.42105^{*}$ & 22 & $122.74351^{*}$ & 21 & $96.43530^{*}$ & $\mathrm{H}$ & 24 & 1.08427 & 23 & 120.43211 & 22 & 180.12133 \\
\hline C & 26 & 1.40940 & 25 & $120.42310^{*}$ & 22 & $184.17002^{*}$ & $\mathrm{H}$ & 28 & 1.08664 & 27 & 118.40112 & 26 & 180.02562 \\
\hline C & 27 & 1.40190 & 26 & 118.64931 & 25 & $180.82942^{*}$ & $\mathrm{H}$ & 29 & 1.08626 & 28 & 120.33044 & 27 & 180.14617 \\
\hline $\mathrm{C}$ & 28 & 1.39189 & 27 & 122.26296 & 26 & 359.76559 & $\mathrm{H}$ & 30 & 1.08710 & 29 & 120.53882 & 28 & 180.02562 \\
\hline $\mathrm{C}$ & 29 & 1.39740 & 28 & 118.76420 & 27 & 359.97438 & $\mathrm{H}$ & 31 & 1.08354 & 30 & 119.21243 & 29 & 179.76689 \\
\hline $\mathrm{C}$ & 30 & 1.39055 & 29 & 120.26866 & 28 & 0.20004 & $\mathrm{H}$ & 34 & 1.08358 & 33 & 119.76961 & 38 & -179.94501 \\
\hline C & 27 & $1.54975^{*}$ & 26 & $123.65876^{*}$ & 25 & $2.19957^{*}$ & $\mathrm{H}$ & 35 & 1.08709 & 34 & 119.20891 & 33 & 179.97438 \\
\hline $\mathrm{C}$ & 25 & $1.41944^{*}$ & 22 & $118.67674^{*}$ & 21 & $274.26073^{*}$ & $\mathrm{H}$ & 36 & 1.08626 & 35 & 120.89328 & 34 & 179.97438 \\
\hline $\mathrm{C}$ & 33 & 1.41195 & 25 & $122.02268^{*}$ & 22 & $361.07643^{*}$ & $\mathrm{H}$ & 37 & 1.08669 & 36 & 119.32083 & 35 & 180.33624 \\
\hline C & 34 & 1.39060 & 33 & 120.94302 & 25 & $180.41148^{*}$ & $\mathrm{H}$ & 41 & 1.08750 & 40 & 120.85913 & 39 & 180.02562 \\
\hline $\mathrm{C}$ & 35 & 1.39745 & 34 & 120.25391 & 33 & 0.10523 & $\mathrm{H}$ & 42 & 1.08715 & 41 & 119.71334 & 40 & 179.97438 \\
\hline $\mathrm{C}$ & 36 & 1.39186 & 35 & 118.78061 & 34 & 359.78995 & $\mathrm{H}$ & 43 & 1.08705 & 42 & 119.74014 & 41 & 179.97438 \\
\hline $\mathrm{C}$ & 37 & 1.40196 & 36 & 122.26388 & 35 & 0.05250 & $\mathrm{H}$ & 44 & 1.08743 & 43 & 120.37776 & 42 & -179.99848 \\
\hline C & 32 & $1.60938^{*}$ & 27 & $100.02180^{*}$ & 26 & $222.74965^{*}$ & $\mathrm{H}$ & 47 & 1.08744 & 46 & 120.72099 & 45 & 179.97438 \\
\hline $\mathrm{C}$ & 39 & 1.40745 & 32 & $112.13108^{*}$ & 27 & $232.40907^{*}$ & $\mathrm{H}$ & 48 & 1.08708 & 47 & 119.73759 & 46 & 180.02562 \\
\hline $\mathrm{C}$ & 40 & 1.39861 & 39 & 120.41622 & 32 & $172.65959^{*}$ & $\mathrm{H}$ & 49 & 1.08711 & 48 & 119.71986 & 47 & 180.02562 \\
\hline $\mathrm{C}$ & 41 & 1.39810 & 40 & 118.87226 & 39 & 0.02562 & $\mathrm{H}$ & 50 & 1.08755 & 49 & 120.25204 & 48 & 179.97980 \\
\hline
\end{tabular}


Table S27: Z-matrix of SpiroAC-TRZ at the TADF maximum with no energy penalty $(\Lambda=0)$.

\begin{tabular}{|c|c|c|c|c|c|c|c|c|c|c|c|c|c|}
\hline & & & & & & & & & & & & & \\
\hline C & 1 & 1.39864 & & & & & & & & & & & \\
\hline $\mathrm{C}$ & 2 & 1.39443 & 1 & 120.16574 & & & & & & & & & \\
\hline $\mathrm{C}$ & 3 & 1.40511 & 2 & 120.36404 & 1 & 0.02562 & & & & & & & \\
\hline $\mathrm{C}$ & 4 & 1.40517 & 3 & 119.15050 & 2 & 359.97438 & $\mathrm{C}$ & 42 & 1.40158 & 41 & 120.58139 & 40 & 359.97438 \\
\hline $\mathrm{C}$ & 5 & 1.39446 & 4 & 120.35081 & 3 & 359.97438 & $\mathrm{C}$ & 43 & 1.40051 & 42 & 120.50910 & 41 & -0.01063 \\
\hline C & 4 & $1.47554^{*}$ & 3 & $111.98727^{*}$ & 2 & $181.37311^{*}$ & $\mathrm{C}$ & 40 & $1.44517^{*}$ & 39 & $108.45973^{*}$ & 44 & $183.12696^{*}$ \\
\hline $\mathrm{N}$ & 7 & 1.34295 & 4 & $110.24980^{*}$ & 3 & $1.19506^{*}$ & $\mathrm{C}$ & 45 & 1.40777 & 40 & $104.91216^{*}$ & 39 & $-0.59160^{*}$ \\
\hline C & 8 & 1.34095 & 7 & 115.86057 & 4 & $180.05717^{*}$ & $\mathrm{C}$ & 46 & 1.39054 & 45 & 120.59798 & 40 & $182.56593^{*}$ \\
\hline $\mathrm{N}$ & 9 & 1.34093 & 8 & 124.28585 & 7 & 0.15796 & $\mathrm{C}$ & 47 & 1.40062 & 46 & 119.00287 & 45 & 359.97438 \\
\hline $\mathrm{C}$ & 10 & 1.34317 & 9 & 115.83276 & 8 & 359.89831 & $\mathrm{C}$ & 48 & 1.40125 & 47 & 120.52755 & 46 & 359.97438 \\
\hline $\mathrm{N}$ & 11 & 1.34192 & 10 & 123.98178 & 9 & -0.04540 & $\mathrm{C}$ & 49 & 1.39825 & 48 & 120.55950 & 47 & 0.03294 \\
\hline C & 11 & $1.66300^{*}$ & 10 & $120.93424^{*}$ & 9 & $180.35447^{*}$ & $\mathrm{H}$ & 1 & 1.08713 & 2 & 120.10348 & 3 & 179.97438 \\
\hline $\mathrm{C}$ & 13 & 1.40515 & 11 & $124.11850^{*}$ & 10 & $359.49472^{*}$ & $\mathrm{H}$ & 2 & 1.08693 & 1 & 120.09391 & 3 & 180.02562 \\
\hline C & 14 & 1.39448 & 13 & 120.35439 & 11 & $179.89436^{*}$ & $\mathrm{H}$ & 3 & 1.08427 & 2 & 120.59577 & 1 & 180.02562 \\
\hline C & 15 & 1.39867 & 14 & 120.16896 & 13 & 359.97438 & $\mathrm{H}$ & 5 & 1.08430 & 4 & 119.02626 & 3 & 179.97438 \\
\hline $\mathrm{C}$ & 16 & 1.39868 & 15 & 119.79481 & 14 & 0.02562 & $\mathrm{H}$ & 6 & 1.08694 & 5 & 119.73537 & 4 & 180.02562 \\
\hline $\mathrm{C}$ & 17 & 1.39438 & 16 & 120.17167 & 15 & 0.02562 & $\mathrm{H}$ & 14 & 1.08428 & 13 & 119.03859 & 18 & 179.95982 \\
\hline $\mathrm{C}$ & 9 & $1.56142^{*}$ & 8 & $114.34246^{*}$ & 7 & $181.06750^{*}$ & $\mathrm{H}$ & 15 & 1.08693 & 14 & 119.73768 & 13 & 179.97438 \\
\hline C & 19 & 1.40442 & 9 & $116.94103^{*}$ & 8 & $360.76368^{*}$ & $\mathrm{H}$ & 16 & 1.08713 & 15 & 120.09936 & 14 & 180.02562 \\
\hline $\mathrm{C}$ & 20 & 1.39361 & 19 & 120.44951 & 9 & $179.87608^{*}$ & $\mathrm{H}$ & 17 & 1.08694 & 16 & 120.08859 & 15 & 180.02562 \\
\hline $\mathrm{C}$ & 21 & 1.39882 & 20 & 120.21264 & 19 & 359.97438 & $\mathrm{H}$ & 18 & 1.08428 & 17 & 120.62263 & 16 & 179.97438 \\
\hline C & 22 & 1.39970 & 21 & 119.67264 & 20 & 0.15218 & $\mathrm{H}$ & 20 & 1.08418 & 19 & 119.09929 & 24 & -179.99032 \\
\hline C & 23 & 1.39353 & 22 & 120.16856 & 21 & 359.85828 & $\mathrm{H}$ & 21 & 1.08639 & 20 & 120.54881 & 19 & 180.13849 \\
\hline $\mathrm{N}$ & 22 & $1.36424^{*}$ & 21 & $119.09386^{*}$ & 20 & $185.15793^{*}$ & $\mathrm{H}$ & 23 & 1.08648 & 22 & 119.31321 & 21 & 179.97438 \\
\hline C & 25 & $1.42155^{*}$ & 22 & $123.85158^{*}$ & 21 & $96.64759^{*}$ & $\mathrm{H}$ & 24 & 1.08427 & 23 & 120.43211 & 22 & 180.12133 \\
\hline $\mathrm{C}$ & 26 & 1.40940 & 25 & $120.17878^{*}$ & 22 & $183.92301^{*}$ & $\mathrm{H}$ & 28 & 1.08664 & 27 & 118.40112 & 26 & 180.02562 \\
\hline $\mathrm{C}$ & 27 & 1.40190 & 26 & 118.64931 & 25 & $180.19737^{*}$ & $\mathrm{H}$ & 29 & 1.08626 & 28 & 120.33044 & 27 & 180.14617 \\
\hline C & 28 & 1.39189 & 27 & 122.26296 & 26 & 359.76559 & $\mathrm{H}$ & 30 & 1.08710 & 29 & 120.53882 & 28 & 180.02562 \\
\hline $\mathrm{C}$ & 29 & 1.39740 & 28 & 118.76420 & 27 & 359.97438 & $\mathrm{H}$ & 31 & 1.08354 & 30 & 119.21243 & 29 & 179.76689 \\
\hline $\mathrm{C}$ & 30 & 1.39055 & 29 & 120.26866 & 28 & 0.20004 & $\mathrm{H}$ & 34 & 1.08358 & 33 & 119.76961 & 38 & -179.94501 \\
\hline C & 27 & $1.54636^{*}$ & 26 & $124.27689^{*}$ & 25 & $2.51236^{*}$ & $\mathrm{H}$ & 35 & 1.08709 & 34 & 119.20891 & 33 & 179.97438 \\
\hline C & 25 & $1.41821^{*}$ & 22 & $119.59668^{*}$ & 21 & $275.55572^{*}$ & $\mathrm{H}$ & 36 & 1.08626 & 35 & 120.89328 & 34 & 179.97438 \\
\hline $\mathrm{C}$ & 33 & 1.41195 & 25 & $122.43565^{*}$ & 22 & $360.93879^{*}$ & $\mathrm{H}$ & 37 & 1.08669 & 36 & 119.32083 & 35 & 180.33624 \\
\hline $\mathrm{C}$ & 34 & 1.39060 & 33 & 120.94302 & 25 & $179.81468^{*}$ & $\mathrm{H}$ & 41 & 1.08750 & 40 & 120.85913 & 39 & 180.02562 \\
\hline C & 35 & 1.39745 & 34 & 120.25391 & 33 & 0.10523 & $\mathrm{H}$ & 42 & 1.08715 & 41 & 119.71334 & 40 & 179.97438 \\
\hline $\mathrm{C}$ & 36 & 1.39186 & 35 & 118.78061 & 34 & 359.78995 & $\mathrm{H}$ & 43 & 1.08705 & 42 & 119.74014 & 41 & 179.97438 \\
\hline $\mathrm{C}$ & 37 & 1.40196 & 36 & 122.26388 & 35 & 0.05250 & $\mathrm{H}$ & 44 & 1.08743 & 43 & 120.37776 & 42 & -179.99848 \\
\hline C & 32 & $1.65550^{*}$ & 27 & $97.68815^{*}$ & 26 & $219.33790^{*}$ & $\mathrm{H}$ & 47 & 1.08744 & 46 & 120.72099 & 45 & 179.97438 \\
\hline C & 39 & 1.40745 & 32 & $111.98606^{*}$ & 27 & $225.06535^{*}$ & $\mathrm{H}$ & 48 & 1.08708 & 47 & 119.73759 & 46 & 180.02562 \\
\hline C & 40 & 1.39861 & 39 & 120.41622 & 32 & $173.05428^{*}$ & $\mathrm{H}$ & 49 & 1.08711 & 48 & 119.71986 & 47 & 180.02562 \\
\hline $\mathrm{C}$ & 41 & 1.39810 & 40 & 118.87226 & 39 & 0.02562 & $\mathrm{H}$ & 50 & 1.08755 & 49 & 120.25204 & 48 & 179.97980 \\
\hline
\end{tabular}


Table S28: Z-matrix of SpiroAC-TRZ at the TADF maximum $(\Lambda=0)$ after the relaxation of the hydrogen atoms and the aromatic ring systems.

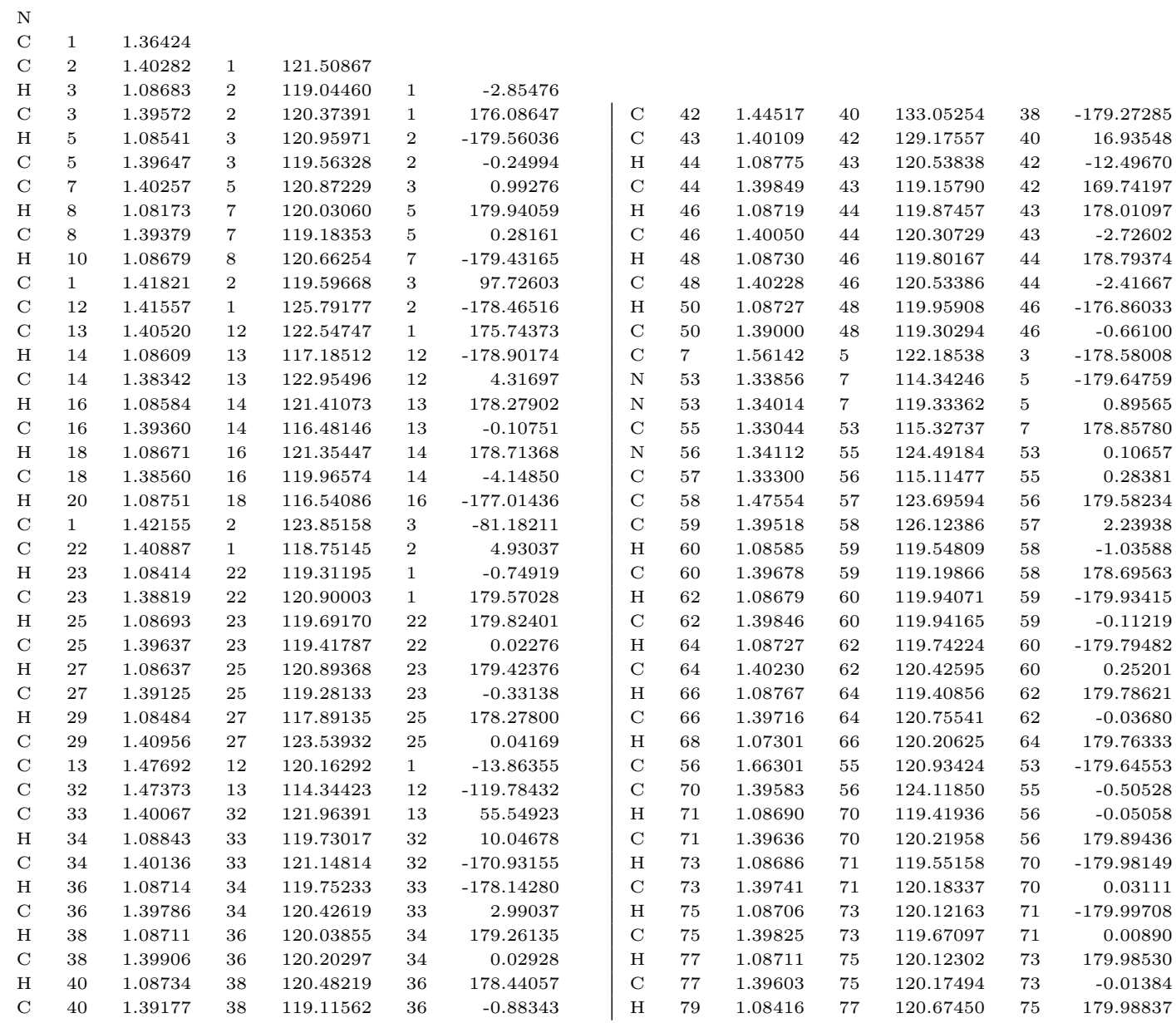




\section{References}

(1) Nelder, J. A.; Mead, R. A Simplex Method for Function Minimization. Comput. J. $1965,7,308-313$.

(2) Marcus, R. A. On the Theory of Oxidation-Reduction Reactions Involving Electron Transfer. I. J. Chem. Phys. 1956, 24, 966-978.

(3) Marcus, R. A. Chemical and Electrochemical Electron-Transfer Theory. Annu. Rev. Phys. Chem. 1964, 15, 155-196.

(4) Beljonne, D.; Shuai, Z.; Pourtois, G.; Brédas, J.-L. Spin-Orbit Coupling and Intersystem Crossing in Conjugated Polymers: A Configuration Interaction Description. J. Phys. Chem. A 2001, 105, 3899-3907.

(5) Schmidt, K.; Brovelli, S.; Coropceanu, V.; Beljonne, D.; Cornil, J.; Bazzini, C.; Caronna, T.; Tubino, R.; Meinardi, F.; Shuai, Z. et al. Intersystem Crossing Processes in Nonplanar Aromatic Heterocyclic Molecules. J. Phys. Chem. A 2007, 111, 10490-10499.

(6) Samanta, P. K.; Kim, D.; Coropceanu, V.; Brédas, J.-L. Up-Conversion Intersystem Crossing Rates in Organic Emitters for Thermally Activated Delayed Fluorescence: Impact of the Nature of Singlet vs Triplet Excited States. J. Am. Chem. Soc. 2017, 139, 4042-4051.

(7) Vosko, S. H.; Wilk, L.; Nusair, M. Accurate spin-dependent electron liquid correlation energies for local spin density calculations: a critical analysis. Can. J. Phys. 1980, 58, $1200-1211$.

(8) Becke, A. D. Density-functional exchange-energy approximation with correct asymptotic behavior. Phys. Rev. A 1988, 38, 3098-3100. 
(9) Lee, C.; Yang, W.; Parr, R. G. Development of the Colle-Salvetti correlation-energy formula into a functional of the electron density. Phys. Rev. B 1988, 37, 785-789.

(10) Becke, A. D. Density-functional thermochemistry. III. The role of exact exchange. J. Chem. Phys. 1993, 98, 5648-5652.

(11) Stephens, P. J.; Devlin, F. J.; Chabalowski, C. F.; Frisch, M. J. Ab Initio Calculation of Vibrational Absorption and Circular Dichroism Spectra Using Density Functional Force Fields. J. Phys. Chem. 1994, 98, 11623-11627.

(12) Kim, K.; Jordan, K. D. Comparison of Density Functional and MP2 Calculations on the Water Monomer and Dimer. J. Phys. Chem. 1994, 98, 10089-10094.

(13) Hariharan, P. C.; Pople, J. A. The influence of polarization functions on molecular orbital hydrogenation energies. Theor. Chim. Acta 1973, 28, 213-222.

(14) Krishnan, R.; Binkley, J. S.; Seeger, R.; Pople, J. A. Self-consistent molecular orbital methods. XX. A basis set for correlated wave functions. J. Chem. Phys. 1980, 72, $650-654$.

(15) Francl, M. M.; Pietro, W. J.; Hehre, W. J.; Binkley, J. S.; Gordon, M. S.; DeFrees, D. J.; Pople, J. A. Self-consistent molecular orbital methods. XXIII. A polarization-type basis set for second-row elements. J. Chem. Phys. 1982, r7, 3654-3665.

(16) Clark, T.; Chandrasekhar, J.; Spitznagel, G. W.; Schleyer, P. V. R. Efficient diffuse function-augmented basis sets for anion calculations. III. The $3-21+\mathrm{G}$ basis set for first-row elements, Li-F. J. Comput. Chem. 1983, 4, 294-301.

(17) Shao, Y.; Gan, Z.; Epifanovsky, E.; Gilbert, A. T. B.; Wormit, M.; Kussmann, J.; Lange, A. W.; Behn, A.; Deng, J.; Feng, X. et al. Advances in molecular quantum chemistry contained in the Q-Chem 4 program package. Mol. Phys. 2015, 113, 184215. 
(18) Perdew, J. P.; Burke, K.; Ernzerhof, M. Generalized Gradient Approximation Made Simple. Phys. Rev. Lett. 1996, 77, 3865-3868.

(19) Perdew, J. P.; Ernzerhof, M.; Burke, K. Rationale for mixing exact exchange with density functional approximations. J. Chem. Phys. 1996, 105, 9982-9985.

(20) Adamo, C.; Barone, V. Toward reliable density functional methods without adjustable parameters: The PBE0 model. J. Chem. Phys. 1999, 110, 6158-6170.

(21) Zhao, Y.; Truhlar, D. G. The M06 suite of density functionals for main group thermochemistry, thermochemical kinetics, noncovalent interactions, excited states, and transition elements: two new functionals and systematic testing of four M06-class functionals and 12 other functionals. Theor. Chem. Acc. 2008, 120, 215-241.

(22) Rohrdanz, M. A.; Herbert, J. M. Simultaneous benchmarking of ground- and excitedstate properties with long-range-corrected density functional theory. J. Chem. Phys. 2008, 129, 034107.

(23) Henderson, T. M.; Janesko, B. G.; Scuseria, G. E. Generalized gradient approximation model exchange holes for range-separated hybrids. J. Chem. Phys. 2008, 128, 194105.

(24) Salzner, U.; Baer, R. Koopmans springs to life. J. Chem. Phys 2009, 131, 231101.

(25) Cancs, E.; Mennucci, B.; Tomasi, J. A new integral equation formalism for the polarizable continuum model: Theoretical background and applications to isotropic and anisotropic dielectrics. J. Chem. Phys. 1997, 107, 3032-3041.

(26) Chipman, D. M. Reaction field treatment of charge penetration. J. Chem. Phys. 2000, 112, 5558-5565.

(27) Cancs, E.; Mennucci, B. Comment on Reaction field treatment of charge penetration [J. Chem. Phys. 112, 5558 (2000)]. J. Chem. Phys. 2001, 114, 4744-4745. 
(28) Mewes, J.-M.; You, Z.-Q.; Wormit, M.; Kriesche, T.; Herbert, J. M.; Dreuw, A. Experimental Benchmark Data and Systematic Evaluation of Two a Posteriori, PolarizableContinuum Corrections for Vertical Excitation Energies in Solution. The Journal of Physical Chemistry A 2015, 119, 5446-5464, PMID: 25629414.

(29) Marchal, J.; Lapp, C. Dielectric properties of polymethyl methacrylate in dilute solution previous results. J. Polym. Sci. 1958, 27, 571-573.

(30) Hanwell, M. D.; Curtis, D. E.; Lonie, D. C.; Vandermeersch, T.; Zurek, E.; Hutchison, G. R. Avogadro: an advanced semantic chemical editor, visualization, and analysis platform. J. Cheminformatics 2012, 4, 17.

(31) Martin, R. L. Natural transition orbitals. J. Chem. Phys. 2003, 118, 4775-4777.

(32) Bode, B. M.; Gordon, M. S. MacMolPlt: a graphical user interface for GAMESS. J. Mol. Graph. Model. 1998, 16, 133-8. 
